\title{
LINE VORTICES IN THE U(1) - HIGGS MODEL
}

\author{
TRISTAN RIVIĖRE
}

\begin{abstract}
For a given U(1)-bundle $E$ over $M=\mathbb{R}^{3} \backslash\left\{x_{1}, \ldots, x_{n}\right\}$, where the $x_{i}$ are $n$ distinct points of $\mathbb{R}^{3}$, we minimise the U(1)-Higgs action and we make an asymptotic analysis of the minimizers when the coupling constant tends to infinity. We prove that the curvature (= magnetic field) converges to a limiting curvature that we give explicitely and which is singular along line vortices which connect the $x_{i}$. This work is the three dimensional equivalent of previous works in dimension two (see [3] and [4]). The results presented here were announced in [12].
\end{abstract}

\section{INTRODUCTION}

Consider $n$ distinct points $\left\{x_{1}, \ldots, x_{n}\right\}$ in $\mathbb{R}^{3}$ and $E$ a $U(1)$-bundle over $M_{\bar{x}}=\mathbb{R}^{3} \backslash\left\{x_{1}, \ldots, x_{n}\right\}$.

Recall that such a bundle is uniquely determined by its first Chern class $c_{1}(E) \in H^{2}\left(M_{\bar{x}}, \mathbb{Z}\right)=\mathbb{Z} \oplus \ldots \oplus \mathbb{Z}$ (n summands), that is $n$ integers $d_{1}, \ldots, d_{n}$ which correspond to the integrals of $c_{1}(E)$ on small spheres surrounding the points $x_{1}, \ldots, x_{n}$. In other words $E$ is uniquely determined by a curvature $h_{0} \in H^{2}\left(M_{\bar{x}}\right)$ on $E$ and for such a curvature one has

$$
\frac{1}{2 \pi} \int_{S} h_{0}=\sum_{i: x_{i} \in \operatorname{Int}(S)} d_{i}
$$

where $S$ is any closed, oriented surface of $\mathbb{R}^{3}$ enclosing some of the points $x_{i}$ in its interior, $\operatorname{Int}(S)$.

We suppose that $\forall i \quad d_{i} \neq 0$ and we make the following neutrality hypothesis

$$
\sum_{i=1 \ldots n} d_{i}=0 .
$$

Under the previous hypothesis, we can write the sequence of points $\left\{x_{i}, \ldots, x_{n}\right\}$ and the corresponding degrees $\left\{d_{1}, \ldots, d_{n}\right\}$ in the form $\left\{P_{1}, \ldots, P_{k}, N_{1}, \ldots, N_{k}\right\}$ where the $P_{i}\left(\operatorname{resp} . N_{i}\right)$ correspond to the $x_{i}$ having positive (resp. negative) integer $d_{i}$ and are repeated as many time as to the multiplicity $\left|d_{i}\right|$.

This paper deals with a variational problem suggested in [8] by J. Fröhlich and M. Struwe. For any section $\varphi$ of the $\mathbb{C}$-line bundle associated to $E$ and

Centre de Mathématiques et de Leurs Applications, CNRS, URA 1611, Ecole Normale Supérieure de Cachan, 61 Avenue du Président Wilson, 94235 Cachan Cedex, France.

Received by the journal October 3, 1995. Accepted for publication March 8, 1996. 
any connection $\tilde{A}$ on $E$, consider the $U(1)-$ Higgs action

$$
S_{\lambda}(\varphi, \tilde{A})=\int_{M_{\bar{x}}}\left|\nabla_{\tilde{A}} \varphi\right|^{2}+|d \tilde{A}|^{2}+\frac{\lambda}{2}\left(1-|\varphi|^{2}\right)^{2},
$$

where $\nabla_{\tilde{A}} \varphi$ is the covariant derivative of $\varphi$ relative to $\tilde{A}, d \tilde{A}$ is the curvature of $\tilde{A}$ and $V(|\varphi|)=\frac{\lambda}{2}\left(1-|\varphi|^{2}\right)^{2}$ is the Higgs potential depending on $|\varphi|$, which, from a mathematical point of view, could be replaced, in the remain of the paper, by any "reasonable" potential on $|\varphi|$ (see [7] part 3) which forces $|\varphi|$ to be close to 1 . The aim is to minimize $S_{\lambda}(\varphi, \tilde{A})$ and to describe the minimizers.

The words used above (section, connection, covariant derivative...) come from geometry but there are also parallel words coming from abelian gauge theory in physics, that we will mix with the previous ones, to designate the same objects. We will also say that $\varphi$ is a scalar (Higgs) field, $\tilde{A}$ is the gauge field (or vector potential), $d \tilde{A}$ is the magnetic field. We will also called $|\varphi|$ the density of the scalar field and $\left(i \varphi, \nabla_{A} \varphi\right)$ the current where $($,$) is the$ usual scalar product on each fiber $\mathbb{C}$ and $i \varphi$ the $\frac{\pi}{2}$ rotation of $\varphi$ on the oriented fibre.

As it is pointed out in $[8]$ for $\left|d_{i}\right| \neq 0 \quad S(\varphi, \tilde{A})=+\infty$, in fact for any $i \in[1, n]$ and $r$ sufficiently small

$$
\int_{\partial B_{r}\left(x_{i}\right)} d \tilde{A}=2 \pi d_{i}
$$

thus by Cauchy-Schwartz inequality

$$
\frac{\pi\left|d_{i}\right|^{2}}{2 r^{2}} \leqslant \int_{\partial B_{r}\left(x_{i}\right)}|d \tilde{A}|^{2}
$$

integrating (1. 1) in $r$ we get $\int|d \tilde{A}|^{2}=+\infty$.

Thus we will adopt the renormalisation proposed in [8].

Consider the following closed 2-form on $M_{\bar{x}}$

$$
h_{0}=-\frac{1}{2} \sum_{i=1, \ldots, n} d_{i} \quad * d\left(\frac{1}{\left|x-x_{i}\right|}\right) .
$$

Where $*$ denote the Hodge operator. $h_{0}$ is a curvature on $E$ and it verifies the following equations

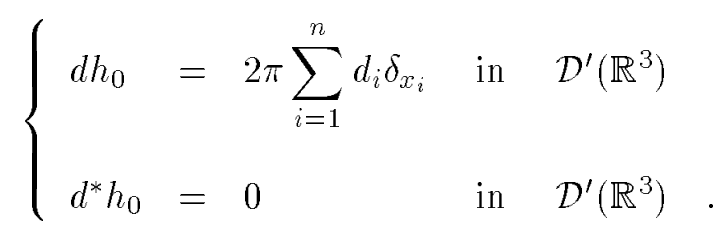

where $d^{*}=* d *$. $h_{0}$ will be called the reference curvature (or magnetic field). Consider a connection $\tilde{A}$ on $E$ and $\tilde{h}=d \tilde{A}$ it's curvature, we denote by $h$ the difference $h=\tilde{h}-h_{0}$. Formally since $d h=0$ in $\mathbb{R}^{3} \backslash\left\{x_{1}, \ldots, x_{n}\right\}$ and $\int_{\partial \Omega} h . \nu=0$ for any regular bounded domain $\Omega$ of $\mathbb{R}^{3}$, thus there exists a Esaim : CoCv June 1996, Vol.1, PP. 77-167 
global 1-form $A$ on $\mathbb{R}^{3}$ such that $h=d A$ and

$$
\begin{aligned}
\int_{\mathbb{R}^{3}}\left(\tilde{h}-h_{0}\right) \cdot h_{0}=\int_{\mathbb{R}^{3}} h \cdot h_{0} & =\int_{\mathbb{R}^{3}} d A \wedge * h_{0} \\
& =-\int_{\mathbb{R}^{3}} A \wedge d * h_{0} \\
& =0
\end{aligned}
$$

(The previous affirmations and equalities could be justified rigourously by assuming some regularity on $\tilde{h}$ in the neighborhood of the $x_{i}$ and infinity. For instance suppose $\tilde{h} \in L^{p}\left(\mathbb{R}^{3}\right)$ for some $1<p<\frac{3}{2}$ and $d^{*} \tilde{h} \in L^{q}\left(\mathbb{R}^{3}\right)$ for some $q>\frac{3}{2}$. This would imply $d \tilde{h}=2 \pi \sum_{i=1}^{n} d_{i} \delta_{x_{i}}$ in $\mathcal{D}^{\prime}\left(\mathbb{R}^{3}\right)$ and the existence of $A \in W_{l o c}^{1, s}\left(\mathbb{R}^{3}\right)$ for some $s>3$ such that $h=d A$ in $\mathcal{D}^{\prime}\left(\mathbb{R}^{3}\right)$ ).

In view of $(1.4)$ we deduce that, by considering $\int_{M_{\bar{x}}}\left|\tilde{h}-h_{0}\right|^{2}$ instead of $\int_{M_{\bar{x}}}|\tilde{h}|^{2}$, on one hand we are substracting the "same infinite quantity" $\int_{M_{\bar{x}}}\left|h_{0}\right|^{2}$ independent of $\tilde{A}$ and, on the other hand, $\int_{M_{\bar{x}}}\left|\tilde{h}-h_{0}\right|^{2}$ has no reason, any more, to be infinite (take $\tilde{h}=h_{0} !$ ).

Thus we are tempted to replace the classical Higg's action by the following one

$$
\tilde{S}_{\lambda}(\varphi, \tilde{A})=\int_{M_{\bar{x}}}\left|\nabla_{\tilde{A}} \varphi\right|^{2}+\left|d \tilde{A}-h_{0}\right|^{2}+\frac{\lambda}{2}\left(1-|\varphi|^{2}\right)^{2} .
$$

In view of formulating a well posed variational problem we introduce a finite covering $U_{\alpha}$ of $\mathbb{R}^{3} \backslash\left\{x_{1}, \ldots, x_{n}\right\}$ in each open set of which we can trivialize the bundle. The transition functions are choosen to be regular and we introduce the following set of minimisation

$$
V=\left\{\begin{array}{l}
\left(\varphi_{\alpha}, \tilde{A}_{\alpha}\right) \in H_{l o c}^{1}\left(U_{\alpha}, \mathbb{C}\right) \times H_{l o c}^{1}\left(U_{\alpha} ; \mathbb{R}^{3}\right) \\
\text { s.t. on } U_{\alpha} \cap U_{\beta} \quad \varphi_{\alpha}=g_{\alpha \beta} \varphi_{\beta} \text { and } i \tilde{A}_{\alpha}=g_{\alpha \beta}^{-1} d g_{\alpha \beta}+i \tilde{A}_{\beta} \\
\tilde{S}_{\lambda}(\varphi, \tilde{A})<+\infty
\end{array}\right\}
$$

We have the following theorem which was already established in [8] and whose proof is essentially based on the choice of a good gauge which makes the functional coercive.

THEOREM 1.1.

$$
\min _{(\varphi, \tilde{A}) \in V} \tilde{S}_{\lambda}(\varphi, \tilde{A}) \text { is achieved }
$$

After having given a proof of theorem 1.1, in the remains of the paper, we will follow the approach of [2] and [4], that is, we will make the parameter $\lambda=\frac{1}{\varepsilon}$ tend to infinity in view of forcing the density $|\varphi|$ to be close to 1 .

The problem is that we cannot expect, for topological reasons, to find a section $\varphi$ of the previous non trivial bundle $E$ taking it's values in the unit sphere of each fiber. This occurs only out of lines which connect the points $x_{i}$ according to their multiplicity. The problem is similar to the one in dimension 2 , here in dimension $3 \varphi$ necessarily has to have a degree around those lines which is independent of the local trivialisation and this will makes the energy tend to infinity. That is the main difficulty that one encounters when one tries to understand the behaviour of the mininimizers as $\lambda$ tends to infinity. The different quantities seem to diverge and one has to work 
harder to get a norm for which something will remain bounded and converge in some space.

The notation $\tilde{S}_{\lambda}(\varphi, \tilde{A})$ is used in [8] where this variational problem was first introduced but, since we make the parameter $\lambda$ tend to infinity, to be consistent with [4], the energy denoted by $\tilde{S}_{\lambda}(\varphi, \tilde{A})$ becomes $G_{\varepsilon}(\varphi, \tilde{A})$ where $\varepsilon=\frac{1}{\sqrt{\lambda}}$.

In fact, as we will see later, the scale $\varepsilon$ will be much more relevant than $\lambda$.

Before we state our main result, we need to introduce the notion of minimal connection already used in [6]. Let $\mathcal{S}_{k}$ be the set of all the permutations in $\{1, \ldots, k\}$. The following quantity $L$ is called minimal connection of the sequence $\left(x_{i}, d_{i}\right)_{i=1, \ldots, n}$

$$
L=\min _{\sigma \in \mathcal{S}_{k}} \sum_{i=1}^{k}\left|P_{i}-N_{\sigma(i)}\right| .
$$

Consider any $\sigma \in S_{k}$ which realizes the previous minimum ( $\sigma$ is perhaps not unique), we will call also minimal connection the 1 dimensional current $I L$ with support in $\bigcup_{i=1}^{k}\left[P_{i}, N_{\sigma(i)}\right]$ oriented by $P_{i}-N_{\sigma(i)}$ and having the integer multiplicity

$$
\theta(x)=\#\left\{i \text { s.t. } x \in\left[P_{i}, N_{\sigma(i)}\right]\right\} .
$$

$L$ is of course the mass of $I L$.

Our main result is the following:

Theorem 1.2. For any sequence $\varepsilon_{n}$ tending to zero and for any sequence $\left(\varphi_{n}, \tilde{A}_{n}\right)$ of minimizers of $G_{\varepsilon_{n}}$ on $V$, one can extract a subsequence, still denoted $\left(\varphi_{n}, \tilde{A}_{n}\right)$, such that, for any $1<p<3 / 2$

$$
h_{n}=d \tilde{A}_{n}-h_{0} \text { strongly converges in } \quad H_{l o c}^{1}\left(\mathbb{R}^{3} \backslash \operatorname{supp} I L\right) \cap W_{l o c}^{1, p}\left(\mathbb{R}^{3}\right)
$$

where $I L$ is a minimal connection associated to the sequence $\left(x_{i}, d_{i}\right)_{i=1, \ldots, n}$. Moreover the limit $h_{\star}$ is the solution of

$$
*\left(-\Delta h_{\star}+h_{\star}+h_{0}\right)=-2 \pi I L
$$

REMARK 1.1. If, for instance, the minimal connection is unique, thus the complete sequence converges.

Remark 1.2. Contrary to the 2 dimensional case, we are not able to give a precise asymptotic expansion of the energy which should be of the form, when $d_{i}= \pm 1$,

$$
G_{\varepsilon n}\left(\varphi_{n}, \tilde{A}_{n}\right)=2 \pi L \log \frac{1}{\varepsilon_{n}}+W\left(x_{i}, d_{i}\right)+0(1) .
$$

Here $W\left(x_{i}, d_{i}\right)$ is a renormalised energy which represents the self interaction of the minimal connection on itself. But this has not the same importance as in the 2-dimensional problem considered in [3] and [4] since, here, we know where the singularities are going to be located and the renormalized energy $W\left(x_{i}, d_{i}\right)$ could be directly computed once we have fixed the $x_{i}$ and $d_{i}$. Esaim : CoCv June 1996, Vol.1, PP. 77-167 
The asymptotic expansion of the minimal energy when all the minimal connections contain a segment with integer multiplicity different from 1 is an interesting question see remarks 2.1 and 2.2.

REMARK 1.3. The analysis developed in this paper could be adapted for solving non-gauge invariant problems in dimension 3 , that is, variational problems for functionals of the form

$$
E_{\varepsilon}(u)=\int_{\Omega \subset \mathbb{R}^{3}}|\nabla u|^{2}+\frac{1}{\varepsilon^{2}}\left(1-|u|^{2}\right)^{2} .
$$

For instance, take $\Omega$ to be a convex domain of $\mathbb{R}^{3}$ and $g_{\varepsilon}$ a boundary condition which simulates the vorticity at the boundary. A particular choice of $g_{\varepsilon}$ could be a " $H^{1}$-approximation" of a map $g_{\star} \quad \partial \Omega \rightarrow S^{1}$ having some degree around different points of $\partial \Omega$ i.e.

$-\left|g_{\varepsilon}\right| \leqslant 1$ on $\partial \Omega$

- $\exists n$ distinct points $\left(a_{1}, \ldots, a_{n}\right)$ of $\partial \Omega$ and $n$ integers $\left(d_{1}, \ldots, d_{n}\right)$ such that

$$
\begin{aligned}
& \sum_{i=1 \ldots n} d_{i}=0 \\
& \left|g_{\varepsilon}\right|=1 \text { on } \partial \Omega \backslash\left(\bigcup_{i=1}^{n} B_{\varepsilon}\left(a_{i}\right) \cap \partial \Omega\right) \\
& \operatorname{deg}\left(g_{\varepsilon}, \partial B_{\varepsilon}\left(a_{i}\right) \cap \partial \Omega\right)=d_{i}
\end{aligned}
$$

- an upper bound for the Dirichlet energy of $g_{\varepsilon}$

$$
\int_{\partial \Omega}\left|\nabla_{\tau} g_{\varepsilon}\right|^{2} \leqslant\left(2 \pi \sum_{i=1}^{n} d_{i}^{2}\right) \log \frac{1}{\varepsilon}+K .
$$

One can prove that, given $\varepsilon_{n}$ tending to zero, from a sequence $u_{\varepsilon_{n}}$ of minimizers of $E_{\varepsilon_{n}}$ among $H_{g_{\varepsilon_{n}}}^{1}(\Omega, \mathbb{C})$ one can extract a subsequence which converges in $H_{l o c}^{1}(\bar{\Omega} \backslash S$ upp $I)$ to an harmonic map $u$ into $S^{1}$, where $I L$ is a minimal connection between the $\left(a_{i}, d_{i}\right)$ and the degree of $u$ arround the different segments of $I L$ corresponds to the multiplicity of the minimal connection along its segments. Moreover, if the minimal connection is unique, the complete sequence converges. The proof of this theorem can be established by following the main ideas of the proof of theorem 2. For instance one can transpose word by word the $\eta$-compactness lemma (part 3 ) to the non-gauge invariant situation just omitting to write $h, A, A_{0}, h_{0}$ ! The case where $\Omega$ is not convex is more complicated since it may happen that the lines of singularities (i.e. the bad set $\left|u_{\varepsilon}\right| \leqslant 1 / 2$ ) tend to touch $\partial \Omega$ as $\varepsilon \rightarrow 0$.

REMARK 1.4. With some more work, one could establish a stronger convergence than $H^{1}$ away from the line singularities as in the two dimensional case (see [2]).

REMARK 1.5. As in the two dimensional case, the description of the minimizers for $\varepsilon>0$ is much more interesting and also more difficult than the one of the limiting situation (see in dimension 2 for $\varepsilon>0[11],[1], \ldots$ ). In dimension 3, also, we can ask similar question to the ones in dimension 2: Are there several minimizers ? In the particular case of a dipole with multiplicity 1 , is the minimizer axially symmetric around the dipole ? We can ask also questions specific to the 3 dimension: For instance when the multiplicity $d$ of our dipole is no more 1 how does the zero set of $|\varphi|$ look like? We can expect to get $d$ different lines connecting the two poles in a way that has to be described. The configuration of those lines seems to be given by a 
1-dimensional variational problem, similar to the zero dimensional problem in 2 dimensions given by the renormalised energy but depending strongly on $\varepsilon$. What could be the resulting interaction energy between those lines?

The paper is devoted to the proof of theorem 1.2. The organisation of the proof is similar to the one developed in [4], but the dimension 3 induces a lot of new technical difficulties. It is for instance much more complicated to detect and to "catch" lines (which can be a-priori very bad) than a finite number of points.

One of the main differences comes also from the stress-energy tensor (see part 2-5) which contains, in dimension 3, a new term preventing a direct local estimate of the density and its closeness to 1 (see [4] part 3). In dimension 2 this closeness ensured quite directly the convergence except on a finite number of small balls. Here, following ideas and classical methods of the regularity theory for non-linear elliptic problems, we deduce, from the conservation law of the stress-energy tensor, some kind of monotonicity formula which is the main tool for proving what we call an " $\eta$-compactness" lemma, ( with reference to the classical $\varepsilon$-regularity lemma of the above mentioned theory). This $\eta$-compactness lemma says that, if the energy on a ball is sufficiently small compared to the global bound (in $\log \frac{1}{\varepsilon}$ ) established in $2-3$, one can ensure that, in the ball of half radius, the density $|\varphi|$ is close to 1 , this is necessary for being far away from the non-compactness locus (the bad part of $\mathbb{R}^{3} \backslash\left\{x_{i}, \ldots, x_{n}\right\}$ ). From the $\eta$-compactness Lemma 3.1 we deduce, in part 4, that, for any $\alpha<1$ the bad set is contained in $\frac{c(\alpha)}{\varepsilon^{\alpha}}$ balls of radius $\varepsilon^{\alpha}$ and that, in some sense, we can bound the vorticity around this union of balls (this is Lemma 4.1). Having located the bad set in something which looks like a line when $\varepsilon \rightarrow 0\left(\frac{c(\alpha)}{\varepsilon^{\alpha}}\right.$ ball or radius $\left.\varepsilon^{\alpha}\right)$, we finally establish a first bound, independent of $\varepsilon$, which is a bound of the $W^{1, p}$ norm of the magnetic field for any $1<p<3 / 2$ (see Lemma 5.1). This gives us a weak convergence, in $W^{1, p}$, of the magnetic field and Theorem 5.1 describes the limit. In part 6 we work out of the limiting bad set for establishing really the $H^{1}$ compactness, in fact we were not sure that, away from the limiting bad set, we could ensure the closeness to 1 for $|\varphi|$ which, combined with the weak $W^{1, p}$ convergence of the magnetic field, leads directly to the compactness. Here again the main ingredient is the $\eta$-compactness lemma. Finally, in part 7 , we show that the limiting bad set which, for topological reasons, has to connect the different points $x_{i}$ according to the multiplicities $d_{i}$, generates an energy, at the level $\varepsilon$, which is asymptotically greater than $2 \pi \log \frac{1}{\varepsilon}$ times the connection it realizes between the $\left(x_{i}, d_{i}\right)$ minus a positive quantity as small as we want. This fact combined with the initial lower bound established in 2.3 forces the limiting bad set to be a minimal connection between the $\left(x_{i}, d_{i}\right)$. The difficulty in this part is essentially technical, since, the limiting bad set, is a-priori only a 1-dimensional rectifiable current whose support is $\mathcal{H}^{1}$-bounded.

In the appendix we prove some technical and basic Lemmas we use in the paper.

Esaim : CoCv June 1996, Vol.1, PP. 77-167 


\section{Preliminaries and Notations}

\subsection{Choice of a covering and a Coulomb Gauge}

First of all we fix a particular covering of $\mathbb{R}^{3} \backslash\left\{x_{1}, \ldots, x_{n}\right\}$ on which we will be able to trivialize $E$ and that will be used in the remains of the paper in particular in 2.3 for establishing an upper bound for the energy.

Suppose first that the indexing of the $\left(N_{1}, \ldots, N_{k}\right)$ is chosen such that the trivial permutation $(i \mapsto i)$ realizes a minimal connection i.e.

$$
\sum_{i=1}^{k}\left|P_{i}-N_{i}\right|=\min _{\sigma \in S_{k}} \sum_{i=1}^{k}\left|P_{i}-N_{\sigma(i)}\right| .
$$

Suppose now there exists $i$ and $i^{\prime}$ such that $\left[P_{i}, N_{i}\right] \cap\left[P_{i^{\prime}}, N_{i^{\prime}}\right]$ contains more than 1 point.

1st case: $\left[P_{i}, N_{i}\right]=\left[P_{i^{\prime}}, N_{i^{\prime}}\right]$. We do not change the sequence $\left(P_{i}, \ldots, P_{k}, N_{i}, \ldots, N_{k}\right)$.

2nd case: $P_{i}=P_{i^{\prime}}$ but $N_{i} \neq N_{i}^{\prime}$ (or the opposite). Suppose $N_{i} \in\left[P_{i^{\prime}}, N_{i^{\prime}}\right]$ thus we repeat one more time $N_{i}$ in the negative terms and we add $N_{i}$ in the positive term thus $\left[P_{i}, N_{i}\right] \cup\left[P_{i^{\prime}}, N_{I^{\prime}}\right]$ is replaced by $\left[P_{i}, N_{i}\right] \cup\left[P_{i}, N_{i}\right] \cup\left[N_{i}, N_{i^{\prime}}\right]$

3nd case: $\left[P_{i}, N_{i}\right] \subset\left[P_{i^{\prime}}, N_{i^{\prime}}\right]$ with $P_{i} \neq P_{i^{\prime}}$ and $N_{i} \neq N_{i^{\prime}}$.

In the sequence $\left(P_{i}, \ldots, P_{k}, N_{i}, \ldots, N_{k}\right)$ we repeat $P_{i}$ and $N_{i}$ such that

$$
\left[P_{i}, N_{i}\right] \cup\left[P_{i^{\prime}}, N_{i^{\prime}}\right]=\left[P_{i^{\prime}}, P_{i}\right] \cup\left[P_{i} N_{i}\right] \cup\left[P_{i}, N_{i}\right] \cup\left[N_{i}, N_{i^{\prime}}\right]
$$

4th case: $\left[P_{i^{\prime}}, N_{i}\right] \subset\left[P_{i}, N_{i^{\prime}}\right]$ with $P_{i^{\prime}} \neq P_{i}$ and $N_{i^{\prime}} \neq N_{i}$

In the sequence $\left(P_{1}, \ldots, P_{k}, N_{1}, \ldots, N_{k}\right)$ we repeat $P_{i^{\prime}}$ and $N_{i}$ such that

$$
\left[P_{i}, N_{i}\right] \cup\left[P_{i^{\prime}}, N_{i^{\prime}}\right]=\left[P_{i}, P_{i^{\prime}}\right] \cup\left[P_{i^{\prime}}, N_{i^{\prime}}\right] \cup\left[P_{i^{\prime}}, N_{i}\right] \cup\left[N_{i}, N_{i^{\prime}}\right]
$$

Thus in all the cases we can change the sequence $\left(P_{i}, N_{i}\right)_{i=1, \ldots, k}$ and the number $k$ without changing neither the bundle nor the minimal connection in such a way that for $i \neq i^{\prime}\left[P_{i}, N_{i}\right] \cap\left[P_{i^{\prime}}, N_{i^{\prime}}\right]=\emptyset$ or a point or $\left[P_{i}, N_{i}\right]=\left[P_{i^{\prime}}, N_{i^{\prime}}\right]$.

We proceed to a last reindexing by associating to a couple $\left(P_{i}, N_{i}\right)$ the positive integer $n_{i}$ which is the number of times the couple is repeated in $\left(\left(P_{i}, N_{i}\right)\right)_{i=1, \ldots, k}$. The previous sequence is now represented by $\left(\left(P_{i}, N_{i}, n_{i}\right)\right)_{i=1, \ldots, k^{\prime}}$ with $k^{\prime} \leqslant k$. $k^{\prime}$ will be denoted by $k$.

Let $O_{i}$ be the middle point of the segment $\left[P_{i}, N_{i}\right]$, and $C_{i}(r)$ be the union of the two half cones of vertices $P_{i}$ and $N_{i}$ and of base the disk perpendicular to $\left[P_{i}, N_{i}\right]$, of centre $O_{i}$ and radius $r$.

We can choose $r$ sufficiently small such that $\forall i \neq i^{\prime} \quad C_{i}(r) \cap C_{i^{\prime}}(r)=\emptyset$ or $C_{i}(r)=C_{i^{\prime}}(r)$.

Let $U_{i}=C_{i}(r)$ for $i \leqslant k$ and $U_{k+1}=\mathbb{R}^{3} \backslash \cup_{i=1}^{k} \overline{C_{i}\left(\frac{r}{2}\right)} .\left(U_{i}\right)_{i=1, \ldots, k+1}$ realizes a covering of $\mathbb{R}^{3} \backslash\left\{x_{i}, \ldots, x_{n}\right\}$ on each open set $U_{i}$ of which we can make a 
trivialisation of E. By $d \theta_{i}$ we denote the angular 1-form around the axis $\left(P_{i}, N_{i}\right)$ oriented by $P_{i}-N_{i}$.

We will only consider trivialisations of $E$ over the $U_{i}$ whose transition functions $g_{i, k+1}(x) \in S^{1}$ on $U_{i} \cap U_{k+1}=C_{i}(r) \backslash C_{i}(r / 2)$ verify

$$
\forall x \in C_{i}(r) \backslash C_{i}(r / 2) \quad g_{i, k+1}^{-1} d g_{i, k+1}(x)=i n_{i} d \theta_{i}
$$

Suppose we have a trivialisation of $E$ over the $U_{i}$ whose transition functions verify (2. 1) we get all the others (verifying (2.1)) simply by multiplying by $e^{i \psi}$ where $\psi$ is a global function on all of $\mathbb{R}^{3} \backslash\left\{x_{1}, \ldots, x_{n}\right\}$. That is the change of trivialisation we will consider most of the time.

Consider the following 1-forms

$$
\begin{aligned}
A_{k+1}^{0}(x) & =\frac{1}{2} \sum_{i=1 \ldots k} n_{i}\left(\frac{x-N_{i}}{\left|x-N_{i}\right|}-\frac{x-P_{i}}{\left|x-P_{i}\right|}\right) \cdot \frac{P_{i}-N_{i}}{\left|P_{i}-N_{i}\right|} d \theta_{i} \text { in } U_{k+1} \\
A_{j}^{0}(x) & =\frac{1}{2} \sum_{i=1 \ldots k} n_{i}\left(\frac{x-N_{i}}{\left|x-N_{i}\right|}-\frac{x-P_{i}}{\left|x-P_{i}\right|}\right) \cdot \frac{P_{i}-N_{i}}{\left|P_{i}-N_{i}\right|} d \theta_{i} \\
& -n_{j} d \theta_{j} \text { in } U_{j}, \quad j \leqslant k
\end{aligned}
$$

Clearly $A_{l}^{0}(l=1, \ldots k+1)$ is regular in $U_{l}$ and we have

$$
\forall j \leqslant k \quad A_{k+1}^{0}(x)=A_{j}^{0}(x)+n_{j} d \theta_{j} \quad \text { in } \quad U_{k+1} \cap U_{j} .
$$

Moreover one verifies that

$$
\forall l \leqslant k+1 \quad d A_{l}^{0}(x)=h_{0}(x) \quad \text { in } \quad U_{l}
$$

Thus, suppose we have chosen a trivialisation of $E$ over the $U_{i}$ which verifies (2. 1), (2. 2) is the expression in this trivialisation of a connection whose curvature is $h_{0}$.

Consider now a $H^{1}$-connection $\tilde{A}$ on $E$ that is $\tilde{A}_{j} \in H_{l o c}^{1}\left(U_{j}, \mathbb{R}^{3}\right)$ for $j \leqslant k+1$ such that

$$
\tilde{A}_{k+1}(x)=\tilde{A}_{j}(x)+n_{j} d \theta_{j} \quad \text { in } \quad U_{k+1} \cap U_{j} .
$$

Let $\tilde{h}=d \tilde{A}$ in $\mathbb{R}^{3} \backslash\left\{x_{1}, \ldots, x_{n}\right\}$ and suppose $h=\tilde{h}-h_{0} \in L^{2}\left(\mathbb{R}^{3}\right)$. Denote by $A$ the following global 1 -form on $\mathbb{R}^{3}$

$$
A(x)=-\frac{1}{4 \pi} d^{*}\left(h * \frac{1}{|x|}\right)=-\frac{1}{4 \pi} \sum_{k=1}^{3} \int_{\mathbb{R}^{3}} \sum_{l=1}^{3} h_{k l}(y) \frac{x^{l}-y^{l}}{|x-y|^{3}} d y d x_{k}
$$

Since $h \in L^{2}\left(\mathbb{R}^{3}\right), d h \in W^{-1,2}\left(\mathbb{R}^{3}\right)$ and since $\operatorname{supp}(d h) \subset\left\{x_{i}, \ldots, x_{n}\right\}$ we have $d h \equiv 0$ in $\mathcal{D}^{\prime}\left(\mathbb{R}^{3}\right)$. Thus we have

$$
\begin{aligned}
d A(x) & =d d^{*}\left(h *-\frac{1}{4 \pi|x|}\right)+d^{*} d\left(h *-\frac{1}{4 \pi|x|}\right) \\
& =\Delta\left(h *-\frac{1}{4 \pi|x|}\right)=h(x) \quad \text { in } \mathcal{D}^{\prime}\left(\mathbb{R}^{3}\right)
\end{aligned}
$$

This implies

$$
d\left(\tilde{A}-A^{0}-A\right)=0 \quad \text { in } \mathbb{R}^{3} \backslash\left\{x_{1}, \ldots, x_{n}\right\}
$$


where $\tilde{A}-A^{0}$ is a global 1 -form defined by

$$
\tilde{A}-A^{0}(x)=\tilde{A}_{l}(x)-A_{l}^{0}(x) \text { in } U_{l} \text {. }
$$

Since $H_{d R}^{1}\left(\mathbb{R}^{3} \backslash\left\{x_{1}, \ldots, x_{n}\right\}\right)=0$ there exists $\psi \in W_{l o c}^{2,2}\left(\mathbb{R}^{3} \backslash\left\{x_{1}, \ldots, x_{n}\right\} ; \mathbb{R}\right)$ such that

$$
\tilde{A}-A^{0}-A(x)=d \psi(x) \text { in } \mathbb{R}^{3} \backslash\left\{x_{1}, \ldots, x_{n}\right\} .
$$

Thus, if we make the global change of trivialisation by multiplying the fiber over $x$ by $e^{-i \psi(x)}$ the new expression of the connection $\tilde{A}$ is given by

$$
\tilde{A}_{l}^{\prime}(x)=\tilde{A}_{l}-d \psi(x)=A_{l}^{0}(x)+A(x) \quad \text { in } U_{l} .
$$

Consider now the set among which we are going to minimize $G_{\varepsilon}$,

$$
V=\left\{\begin{array}{l}
\left(\varphi_{l}, \tilde{A}_{l}\right) \in H_{l o c}^{1}\left(U_{l}, \mathbb{C}\right) \times H_{l o c}^{1}\left(U_{l}, \mathbb{R}^{3}\right) \text { s.t. } \\
\forall j \leqslant k \varphi_{k+1}=\varphi_{j} e^{i n_{j} \theta_{j}}, \tilde{A}_{k+1}=\tilde{A}_{j}+n_{j} d \theta_{j} \text { in } U_{j} \cap U_{k+1} \\
G_{\varepsilon}(\varphi, \tilde{A})<+\infty
\end{array}\right\}(2.9)
$$

where $e^{i \theta_{j}}$ is the angular coordinate around the axis $\left[P_{j}, N_{j}\right]$ in $C_{j}(r) \backslash C_{j}\left(\frac{r}{2}\right)$ where we have fixed some horizontal direction equal to 1.

Among $V$ we say that $(\varphi, \tilde{A})$ is equivalent to $\left(\varphi^{\prime}, \tilde{A}^{\prime}\right)$ if there exists $\psi \in W_{l o c}^{2,2}\left(\mathbb{R}^{3} \backslash\left\{x_{1}, \ldots, x_{n}\right\}, \mathbb{R}\right)$ such that

$$
\forall 1 \leqslant l \leqslant k+1 \quad \tilde{A}_{l}=\tilde{A}_{l}^{\prime}+d \psi \quad \text { and } \quad \varphi_{l}=e^{i \psi} \varphi_{l}^{\prime} \quad \text { in } \quad U_{l}
$$

Proposition 2.1. Let $(\varphi, \tilde{A}) \in V$, there exists $\left(\varphi^{\prime}, \tilde{A}^{\prime}\right) \in V$ equivalent to $(\varphi, \tilde{A})$ such that

$$
\forall 1 \leqslant l \leqslant k+1 \quad \tilde{A}_{l}^{\prime}(x)=A_{l}^{0}(x)+A(x) \quad \text { in } \quad U_{l}
$$

where $A(x)=-\frac{1}{4 \pi} d^{*}\left(\left(\tilde{h}-h_{0}\right) * \frac{1}{|x|}\right)$ : This is what we call the Coulomb gauge of $\tilde{h}$.

The proposition is proved just above, the name Coulomb gauge comes from the fact that

$$
d^{*} A=0 \text { in } \mathcal{D}^{\prime}\left(\mathbb{R}^{3}\right) .
$$

Moreover one easily verifies that

$$
\forall l \in\{1, \ldots, k\} \quad d^{*} A_{l}^{0}=0 \quad \text { in } \quad \mathcal{D}^{\prime}\left(U_{l}\right) .
$$

Thus

$$
\forall l \in\{1, \ldots, k\} \quad d^{*} \tilde{A}_{l}^{\prime}=0 \quad \text { in } \quad \mathcal{D}^{\prime}\left(U_{l}\right)
$$

\subsection{Existence of minimizers. Proof of theorem 1.1}

In this part we prove the theorem 1 stated in the introduction. More precisely we prove the following,

Theorem 2.3. For any $\varepsilon>0$ the minimum of $G_{\varepsilon}$ among $V$ (defined by (2. $9)$ ) is achieved by, at least, one class of couples $\left(\varphi_{\varepsilon}, \tilde{A}_{\varepsilon}\right)$ (for the relation 2. 10). The $\left(\varphi_{\varepsilon}, \tilde{A}_{\varepsilon}\right)$ verify

$$
-\frac{1}{2} \Delta\left|\varphi_{\varepsilon}\right|^{2}=\frac{1}{\varepsilon^{2}}\left|\varphi_{\varepsilon}\right|^{2}\left(1-\left|\varphi_{\varepsilon}\right|^{2}\right)-\left|\nabla_{\tilde{A}_{\varepsilon}} \varphi_{\varepsilon}\right|^{2} \text { in } \mathcal{D}^{\prime}\left(\mathbb{R}^{3} \backslash\left\{x_{1}, \ldots, x_{n}\right\}\right),(2.14)
$$


and

$$
-d^{*} h_{\varepsilon}=J_{\varepsilon} \quad \text { in } \mathcal{D}^{\prime}\left(\mathbb{R}^{3}\right)
$$

where $J_{\varepsilon}$, the current, is the gauge invariant quantity equal to $\left(i \varphi_{\varepsilon}, d_{\tilde{A}_{\varepsilon}} \varphi_{\varepsilon}\right)$ in $\mathbb{R}^{3} \backslash\left\{x_{1}, \ldots, x_{n}\right\}$. Moreover $\left|\varphi_{\varepsilon}\right|^{2}, J_{\varepsilon}, h_{\varepsilon}$ are regular in $\mathbb{R}^{3} \backslash\left\{x_{1}, \ldots, x_{n}\right\}$.

\section{Proof of theorem 2.3}

Let $\varepsilon$ be a fixed positive real for the remain of the proof. Let $\left(\varphi^{p}, \tilde{A}^{p}\right)$ be a minimizing sequence of $G_{\varepsilon}$. We have

$$
\int_{\mathbb{R}^{3}}\left|h^{p}\right|^{2}=\int_{\mathbb{R}^{3} \backslash\left\{x_{1}, \ldots, x_{n}\right\}}\left|\tilde{h}^{p}-h_{0}\right|^{2}<C \quad \text { indep. of } p .
$$

Thus by classical estimates for Calderon - Zygmund operators we have

$$
\left(\int_{\mathbb{R}^{3}}\left|A^{p}\right|^{6}\right)^{1 / 6}+\left(\int_{\mathbb{R}^{3}}\left|\nabla A^{p}\right|^{2}\right)^{1 / 2} \leqslant C\left(\int_{\mathbb{R}^{3}}\left|h^{p}\right|^{2}\right)^{1 / 2} \leqslant C
$$

where $A^{p}$ is defined by (2.6).

In the class of $(\varphi, \tilde{A})$ consider the Coulomb gauge given by Proposition II.1. For any $l \leqslant k+1$ and $K$ compact set in $U_{l}$, we have

$$
\int_{U_{l} \cap K}\left|\nabla_{\tilde{A}^{p}} \varphi^{p}\right|^{2} \leqslant C
$$

thus

$$
\begin{aligned}
\int_{U_{l} \cap K}\left|\nabla \varphi_{l}^{p}\right|^{2} & \leqslant C+2 \int_{U_{l} \cap K}\left|\tilde{A}_{l}^{p}\right|^{2}\left|\varphi^{p}\right|^{2} \\
& \leqslant C+2 \int_{U_{l} \cap K}\left|\tilde{A}_{l}^{p}\right|^{2}+ \\
& +2\left(\int_{U_{l} \cap K}\left|\tilde{A}_{l}^{p}\right|^{4}\right)^{1 / 2}\left(\int_{U_{l} \cap K}\left(1-\left|\varphi^{p}\right|^{2}\right)^{2}\right)^{1 / 2}
\end{aligned}
$$

Since $A_{l}^{0}$ is regular on $U_{l}, \int_{U_{l} \cap K}\left|A_{l}^{0}\right|^{4}$ is bounded and from (2.15) we deduce that

$$
\int_{U_{l} \cap K}\left|A_{l}^{0}+A^{p}\right|^{4} \leqslant C \quad \text { indep. of } p \quad .
$$

Thus from (2. 18) and (2. 19) it follows that $\varphi_{l}^{p}$ weakly converges in $W_{l o c}^{1,2}\left(U_{l}, \mathbb{C}\right)$ (up to a subsequence) and this is also the case for $\tilde{A}_{l}^{p}=A_{l}^{0}+A^{p}$. By lower semi continuity of the $W^{1,2}$ it is clear that the limit $\left(\varphi_{l, \varepsilon}, A_{l}^{0}+\right.$ $\left.A_{\varepsilon}^{p}\right)_{l=1 \ldots k+1}$ minimizes $G_{\varepsilon}$.

The fact that equations (2. 14) and (2. 15) are verified in $\mathcal{D}^{\prime}\left(\mathbb{R}^{3} \backslash\left\{x_{1}, \ldots, x_{n}\right\}\right)$ and that, moreover, $\left|\varphi_{\varepsilon}\right|^{2}, J_{\varepsilon}$ and $h_{\varepsilon}$ are regular in $\mathbb{R}^{3} \backslash\left\{x_{1}, \ldots, x_{n}\right\}$ is proved in $[10]$. Let us just prove that $(2.15)$ is verified in $\mathcal{D}^{\prime}\left(\mathbb{R}^{3}\right)$.

Let $\Omega$ be a bounded domain of $\mathbb{R}^{3}$, since $h_{\varepsilon} \in L^{2}(\Omega), d^{*} h_{\varepsilon} \in W^{-1,2}(\Omega)$, moreover, since $\int_{\mathbb{R}^{3}}\left(1-\left|\varphi_{\varepsilon}\right|^{2}\right)^{2}<+\infty,\left|\varphi_{\varepsilon}\right| \in L^{4}(\Omega)$ and this implies that Esaim : Cocv June 1996, Vol.1, Pp. 77-167 
the function $J_{\varepsilon}$ on $\mathbb{R}^{3}$ defined by $J_{\varepsilon} \equiv\left(i \varphi_{\varepsilon}, d_{A_{\varepsilon}} \varphi_{\varepsilon}\right)$ in $\mathbb{R}^{3} \backslash\left\{x_{1}, \ldots, x_{n}\right\}$ is in $L^{4 / 3}(\Omega) \subset W^{-1,2}(\Omega)$.

Thus

$$
d^{*} h_{\varepsilon}+J_{\varepsilon} \in W^{-1,2}(\Omega),
$$

and $\operatorname{supp}\left(d^{*} h_{\varepsilon}+J_{\varepsilon}\right) \subset\left\{x_{1}, \ldots, x_{n}\right\}$, so this implies (2. 15).

\subsection{An upPER BOUND FOR THE ENERGY}

In this section we prove the following lemma

LEMma 2.1. For any $\alpha>0$ we have

$$
\min _{V} G_{\varepsilon} \leqslant 2 \pi(L+\alpha) \operatorname{Ln} \frac{1}{\varepsilon}+K(\alpha),
$$

where $L$ is the minimal connection between the $\left(x_{i}, d_{i}\right)$ and $K$ only depends on $\alpha$, not on $\varepsilon$.

Remark 2.6. Contrary to the 2-dim. case, the upper bound for the energy requires the introduction of an external parameter $\alpha$. This is not because the dimension 3 leeds to new technical difficulties, this weaker upper-bound, Compare to the two dimensional one (see proposition 2.5 [4]), is intrinsically linked to the dimension 3 for any minimal connection between the $\left(x_{i}, d_{i}\right)$ containing a segment with integer multiplicity different from 1 .

Consider for instance the case of a dipole $(P, N)$ of charge $d>1$ ie $\left(\left(x_{i}, d_{i}\right)\right)=$ $((P, d),(N,-d))$. The minimal connection is the segment $[P, N]$ with the integer multiplicity $d$, that is $I L=d[|[P, N]|]$ and $L=d|P-N|$. The singular set tends to concentrate along this segment when $\varepsilon \mapsto 0$. Suppose this configuration is adopted for any $\varepsilon>0$. Then the energy would be greater than $2 \pi d^{2}|P-N| \log \frac{1}{\varepsilon}$ ! Thus at the stage $\varepsilon$, what we call the bad set prefer to decompose $d$ distinct tubes between $P$ and $N$ which are close to the segment $[P, N]$ (but not too much so that the interaction energy between them is at most of order $\approx \log \log \varepsilon$ ).

REMARK 2.7. If $\left(x_{i}, d_{i}\right)$ admits a minimal connection which contains no segment of integer multiplicity different from 1, then Lemma 2.1 holds for $\alpha=0$. It seems that this is a minimal connection that the singular set would prefer to adopt.

\section{Proof of Lemma 2.1.}

Let $\alpha>0$ and $\varepsilon>0$. We construct $\left(\varphi_{l}, \tilde{A}_{l}\right)$ in $V$ verifying (2. 20). First of all we construct $|\varphi|$.

For $1 \leqslant i \leqslant k$ consider $n_{i}$ the multiplicity of $\left(P_{i}, N_{i}\right)$ in the minimal connection that we consider and for $1 \leqslant s \leqslant n_{i}$ introduce $L_{i, s}$ to be the following segments.

If $n_{i}=1$, take $L_{i, s}=L_{i, 1}=\left[P_{i}, N_{i}\right]$. and if $n_{i} \neq 1$ we identify the perpendicular plane to $\left(P_{i}, N_{i}\right)$ passing by $O_{i}$, the center of $\left[P_{i}, N_{i}\right]$, with $\mathbb{C}$ and denote by $\left(a_{i, s}\right)_{s=1, \ldots, n_{i}}$ the $n_{i}$-th roots of 1 multiplied by $\alpha$. (we suppose $\alpha<\frac{r}{4}$ in such a way that $a_{i, s} \in C_{i}\left(\frac{r}{2}\right)$ and also for technical reasons which 
will be clear later). Take $L_{i, s}=\left[P_{i}, a_{i, s}\right] \cup\left[a_{i, s}, N_{i}\right]$.

Consider $\tilde{L}$ the following union of segments

$$
\tilde{L}=\cup_{i=1}^{k} \cup_{s=1}^{n_{i}} L_{i s}
$$

Let $\chi$ be the following cut-off function on $\mathbb{R}_{+}$

$$
\chi(x)=x \text { for } x \leqslant 1 \text { and } \chi(x)=1 \text { for } x \geqslant 1 .
$$

Define $|\varphi|(x)=\chi\left(\frac{\operatorname{dist}(x, \tilde{L})}{\varepsilon}\right)$. We have

$$
\left.\int_{\mathbb{R}^{3}}|\nabla| \varphi\right|^{2}+\frac{1}{2 \varepsilon^{2}}\left(1-|\varphi|^{2}\right)^{2} \leqslant \int_{\operatorname{supp}(1-|\varphi|)} \frac{C}{\varepsilon^{2}} \leqslant C,
$$

where $C$ does not depend on $\varepsilon$.

We choose

$$
\forall l \leqslant k+1 \quad \tilde{A}_{l}=A_{l}^{0} \quad \text { in } U_{l} .
$$

We claim that

$$
\sum_{l=1 \ldots k+1} \int_{U_{l}}\left|A_{l}^{0}\right|^{2}<+\infty
$$

It suffices to prove that, if one considers $C_{1}$ the union of the two cones of vertices $p=(0,0+1)$ and $N=(0,0,-1)$ and of base the horizontal unit disk of center 0 and if we take

$$
\begin{gathered}
A_{1}^{0}=\left[\frac{1}{2}\left(\frac{1-z}{|X-P|}+\frac{z+1}{|X-N|}\right)-1\right] \frac{x d y-y d x}{\left(x^{2}+y^{2}\right)} \quad \text { in } C_{1} \\
A_{2}^{0}=\left[\frac{1}{2}\left(\frac{1-z}{|X-P|}+\frac{z+1}{|X-N|}\right)\right] \frac{x d y-y d x}{x^{2}+y^{2}} \quad \text { in } \mathbb{R}^{3} \backslash C_{1}
\end{gathered}
$$

where $X=(x, y, z)$. Then we have

$$
\int_{C_{1}}\left|A_{1}^{0}\right|^{2}+\int_{\mathbb{R}^{3} \backslash C_{1}}\left|A_{2}^{0}\right|^{2}<+\infty
$$

This is verified by a direct computation.

Let $l \leqslant k$, consider an orthonormal basis $\left(\vec{i}_{l}, \vec{j}_{l}, \vec{k}_{l}\right)$ such that $\vec{k}_{l}=\frac{P_{l}-N_{l}}{\left|P_{l}-N_{l}\right|}$. Each horizontal plane is identified with $\mathbb{C}$.

If $n_{l}=1$, take

$$
\varphi_{l}(x)=|\varphi|(x) e^{-i \theta_{l}} \quad \text { in } U_{l}
$$

where $\theta_{l}$ is the polar horizontal angle of $x$ in cylindrical coordinates associated with $\left(\vec{i}_{l}, \vec{j}_{l}, \vec{k}_{l}\right)$.

If $n_{l}>1$. For $0 \leqslant z_{0} \leqslant\left|P_{l}-O_{l}\right|=D_{l}$ (resp. $\left.0 \geqslant z_{0} \geqslant-\left|P_{l}-O_{l}\right|\right)$, denote by $a_{l, s}\left(z_{0}\right)$ the point $a_{l, s}\left(z_{0}\right)=\left\{z=z_{0}\right\} \cap\left[P_{l}, a_{l, s}\right]$ (resp. $a_{l, s}\left(z_{0}\right)=\{z=$ $\left.\left.z_{0}\right\} \cap\left[a_{l, s}, N_{l}\right]\right)$. Take

$$
\varphi_{l}(x, y, z)=|\varphi|(x, y, z) \prod_{s \equiv 1}^{n_{l}}\left(\frac{\xi-\lambda \tilde{a}_{l, s}(z)}{\left|\xi-\lambda \tilde{a}_{l, s}(z)\right|}\right)^{-1} \quad \text { in } U_{l},
$$


where $\xi=x+i y, \tilde{a}_{l, s}(z)$ is the projection on $\{z=0\} \simeq \mathbb{C}$ of $a_{l, s}(z)$ and $\lambda$ is an interpolation function between 0 and 1 verifying $\lambda \equiv 1$ in $C_{l}\left(\frac{r}{4}\right) \lambda \equiv 0$ in $C_{l}\left(\frac{r}{2}\right)$ and $\lambda=0$ outside. Precisely we take

$$
\text { for } 0 \leqslant z \leqslant D_{l} \quad \lambda(x, y, z)=\lambda(|\xi|, z)=2-\frac{4 D_{l}}{D_{l}-z} \frac{|\xi|}{r},
$$

and

$$
\lambda(|\xi|,-z)=\lambda(|\xi|, z)
$$

From (2.24) and (2. 25) we deduce that

$$
\varphi_{k+1}=|\varphi| \quad \text { in } U_{k+1} .
$$

If $n_{l}=1$, we have from (2. 24)

$$
\begin{aligned}
\int_{U_{l}}\left|\nabla \varphi_{l}\right|^{2} & =\left.\int_{U_{l}}|\nabla| \varphi\right|^{2}+\int_{U_{l}}|\varphi|^{2} \frac{1}{\rho^{2}} \rho d \rho d \theta d z \\
& \leqslant C+2 \pi \times 2 \int_{0}^{D_{l}-\varepsilon} \int_{\varepsilon}^{\frac{D_{l}-z}{D_{l}}} \frac{1}{\rho} d \rho d z \\
& \leqslant C+2 \pi\left(2 D_{l}\right) \log \frac{1}{\varepsilon}+4 \pi \int_{0}^{D_{l}-\varepsilon} \log \left[\left(1-\frac{z}{D_{l}}\right) r\right] d z \\
& \leqslant 2 \pi\left|P_{l}-N_{l}\right| \log \frac{1}{\varepsilon}+C,
\end{aligned}
$$

where $C$ does not depend on $\varepsilon$.

If $n_{l}>1$ we write

$$
\int_{U_{l}}\left|\nabla \varphi_{l}\right|^{2}=\int_{C_{l}\left(\frac{r}{4}\right)}\left|\nabla \varphi_{l}\right|^{2}+\int_{U_{l} \backslash C_{l}\left(\frac{r}{4}\right)}\left|\nabla \varphi_{l}\right|^{2} .
$$

One verifies that the second integral on the right hand side of (2.27) can be bounded independently of $\varepsilon$. Indeed, in $U_{l} \backslash\left(C_{l}\left(\frac{r}{4}\right) \cup B_{\varepsilon}\left(P_{l}\right) \cup B_{\varepsilon}\left(N_{l}\right)\right)|\varphi|=1$ and

$$
\begin{aligned}
\left|\nabla \prod_{s=1}^{n_{l}}\left(\frac{\xi-\lambda(|\xi|, z) \tilde{a}_{l s}(z)}{\left|\xi-\lambda(|\xi|, z) \tilde{a}_{l s}(z)\right|}\right)\right| & \leqslant C \sum_{s=1}^{n_{l}} \frac{1}{\left|\xi-\lambda \tilde{a}_{l s}(z)\right|} \\
& \leqslant C \frac{n_{l}}{\frac{r}{4}-\alpha} .
\end{aligned}
$$

Recall that the $\alpha$ that we consider are strictly less than $r / 4$. The first integral of (2.27) can be bounded in the following way

$$
\begin{aligned}
\int_{C_{l}\left(\frac{r}{4}\right)} & \left|\nabla \varphi_{l}\right|^{2} \leqslant\left.\int_{C_{l}\left(\frac{r}{4}\right)}|\nabla| \varphi_{l}\right|^{2}+C \\
& +2 \int_{0}^{D_{l}} d z \int_{B_{2}(z) \backslash U B\left(a_{l, s}(z), \varepsilon\right)}\left|\nabla \prod_{\substack{s=1 \\
\text { ESAIM : COCV JUNE 1996, VoL.1, PP. } 77-167}}^{n_{l}}\left(\frac{\mid \xi-\tilde{a}_{l, s}(z)}{\left|\xi-\tilde{a}_{l, s}(z)\right|}\right)\right|^{2} \quad \text { (2. 28) }
\end{aligned}
$$


where $B_{2}(z)$ denotes the horizontal disk of radius $r \frac{D_{l}-z}{D_{l}}$ and center the point $(0,0, z)$ in $\left(\vec{i}_{l}, \vec{j}_{l}, \vec{k}_{l}\right)$.

We have

$$
\left|\frac{\partial}{\partial z} \prod_{s=1}^{n_{l}}\left(\frac{\xi-\tilde{a}_{l, s}(z)}{\left|\xi-\tilde{a}_{l, s}(z)\right|}\right)\right| \leqslant \sum_{s=1}^{n_{l}}\left|\frac{\partial \tilde{a}_{l, s}}{\partial z}\right| \times \frac{C}{\left|\xi-\tilde{a}_{l, s}(z)\right|}
$$

but

$$
\left|\frac{\partial \tilde{a}_{l, s}}{\partial z}\right| \leqslant C \alpha \text { and } \int_{B_{2}(z) \backslash B\left(a_{l s}(z), \varepsilon\right)} \frac{1}{\left|\xi-\tilde{a}_{l s}(z)\right|^{2}} \leqslant C \log \frac{1}{\varepsilon}
$$

thus we have

$$
\int_{0}^{D_{l}} d z \int_{B_{2}(z) \backslash B\left(a_{l s}(z), \varepsilon\right)}\left|\frac{\partial}{\partial z} \prod_{s=1}^{n_{l}}\left(\frac{\xi-\tilde{a}_{l, s}(z)}{\left|\xi-\tilde{a}_{l, s}(z)\right|}\right)\right|^{2} \leqslant C \alpha^{2} \log \frac{1}{\varepsilon} .
$$

Concerning the horizontal derivatives in the second integral of the right hand side of (2. 28) we use an upper bound given by (4.38) in [7]. Finally one verifies easily that the energy of $\left|\varphi_{l}\right|$ is uniformly bounded, thus we deduce

$$
\int_{C_{l}\left(\frac{r}{4}\right)}\left|\nabla \varphi_{l}\right|^{2} \leqslant 2 \pi n_{l}\left(\left|P_{l}-N_{l}\right|+c \alpha^{2}\right) \log \frac{1}{\varepsilon}+K(\alpha) .
$$

Combining (2. 21), (2. 23), (2. 26) and (2. 31) we get the desired result.

\subsection{Preliminary estimates}

We establish, first, the $L^{\infty}$ bound for $\left|\varphi_{\varepsilon}\right|$.

Lemma 2.2. Let $\left(\varphi_{\varepsilon}, \tilde{A}_{\varepsilon}\right) \in V$ be a minimizer of $G_{\varepsilon}$ then we have

$$
\left|\varphi_{\varepsilon}\right| \leqslant 1 \quad \text { in } \mathbb{R}^{3} \backslash\left\{x_{1}, \ldots, x_{n}\right\} \text {. }
$$

\section{Proof of Lemma 2.2.}

Suppose $\omega=\left\{x \in \mathbb{R}^{3} \backslash\left\{x_{1}, \ldots, x_{n}\right\} /\left|\varphi_{\varepsilon}\right|(x)>1\right\}$ is not empty. Define $\tilde{\varphi}_{\varepsilon}$ in the following way

$$
\tilde{\varphi}_{\varepsilon}=\varphi_{\varepsilon} \quad \text { if }\left|\varphi_{\varepsilon}\right| \leqslant 1
$$

and

$$
\tilde{\varphi}_{\varepsilon}=\varphi_{\varepsilon} /\left|\varphi_{\varepsilon}\right| \quad \text { in } \omega
$$

It is clear that

$$
\int_{\mathbb{R}^{3} \backslash\left\{x_{1}, \ldots, x_{n}\right\}}\left(1-\left|\tilde{\varphi}_{\varepsilon}\right|^{2}\right)^{2}<\int_{\mathbb{R}^{3} \backslash\left\{x_{1}, \ldots, x_{n}\right\}}\left(1-\left|\varphi_{\varepsilon}\right|^{2}\right)^{2} .
$$

Moreover since

$$
\forall x \in \omega \quad\left|\nabla_{\tilde{A}_{\varepsilon}} \tilde{\varphi}_{\varepsilon}\right|^{2}(x)=\left|\nabla_{\tilde{A}_{\varepsilon}} \frac{\varphi_{\varepsilon}}{\left|\varphi_{\varepsilon}\right|}\right|^{2},
$$

and

$$
\forall x \in \omega \quad\left|\nabla_{\tilde{A}_{\varepsilon}} \varphi_{\varepsilon}\right|^{2}(x)=|\nabla| \varphi_{\varepsilon}||^{2}(x)+\left|\nabla_{\tilde{A}_{\varepsilon}} \frac{\varphi_{\varepsilon}}{\left|\varphi_{\varepsilon}\right|}\right|^{2},
$$


we have

$$
\forall x \in \omega \quad\left|\nabla_{\tilde{A}_{\varepsilon}} \tilde{\varphi}_{\varepsilon}\right|(x) \leqslant\left|\nabla_{\tilde{A}_{\varepsilon}} \varphi_{\varepsilon}\right|(x)
$$

The previous inequality combined with (2. 33) imply $G_{\varepsilon}\left(\tilde{\varphi}_{\varepsilon}, \tilde{A}_{\varepsilon}\right)<G_{\varepsilon}\left(\varphi_{\varepsilon}, \tilde{A}_{\varepsilon}\right)$, contradiction.

We establish, now, preliminary estimates for $A_{\varepsilon}$ and $h_{\varepsilon}$

Lemma 2.3. Let $\left(\varphi_{\varepsilon}, \tilde{A}_{\varepsilon}\right) \in V$ be a minimizer of $G_{\varepsilon}, h_{\varepsilon}=d \tilde{A}_{\varepsilon}-h^{0}$ and $A_{\varepsilon}$ defined by (2.6), we have for $\varepsilon$ sufficiently small

$$
\begin{gathered}
\left(\int_{\mathbb{R}^{3}}\left|A_{\varepsilon}\right|^{6}\right)^{1 / 3}+\int_{\mathbb{R}^{3}}\left|\nabla A_{\varepsilon}\right|^{2} \leqslant C \int_{\mathbb{R}^{3}} h_{\varepsilon}^{2} \leqslant C \log \frac{1}{\varepsilon}, \\
\int_{\mathbb{R}^{3}}\left|\nabla h_{\varepsilon}\right|^{2} \leqslant \int_{\mathbb{R}^{3}}\left|\nabla^{2} A_{\varepsilon}\right|^{2} \leqslant C \log \frac{1}{\varepsilon}, \\
\forall r \leqslant 1 \quad \forall x \in \mathbb{R}^{3} \quad \frac{1}{r^{2}} \int_{B_{r}(x)} h_{\varepsilon}^{2} \leqslant C \log \frac{1}{\varepsilon}, \\
\forall r \leqslant 1 \quad \forall x \in \mathbb{R}^{3} \quad \frac{1}{r} \int_{\partial B_{r}(x)} h_{\varepsilon}^{2} \leqslant C \log \frac{1}{\varepsilon}
\end{gathered}
$$

and

$$
\begin{gathered}
\forall x, y \in \mathbb{R}^{3} \text { s.t. }|x-y| \leqslant 1 \\
\left|A_{\varepsilon}(x)-A_{\varepsilon}(y)\right| \leqslant C\left(\log \frac{1}{\varepsilon}\right)^{1 / 2}|x-y|^{1 / 2} .
\end{gathered}
$$

\section{Proof of Lemma 2.3.}

(2. 35) comes from (2. 20) and classical estimates on Calderon-Zygmund operators.

From (2. 15) we know that

$$
-d^{*} h_{\varepsilon}=J_{\varepsilon} \quad \text { in } \mathcal{D}^{\prime}\left(\mathbb{R}^{3}\right)
$$

In the other hand, by definition, we have

$$
A_{\varepsilon}=-\frac{1}{4 \pi} \frac{1}{|x|} * d^{*} h_{\varepsilon}=+\frac{1}{4 \pi} \frac{1}{|x|} * J_{\varepsilon}
$$

From (2. 20) and (2. 32) we have $J_{\varepsilon} \in L^{2}\left(\mathbb{R}^{3}\right)$ and

$$
\int_{\mathbb{R}^{3}}\left|J_{\varepsilon}\right|^{2}=\int_{\mathbb{R}^{3}}\left|\left(i \varphi, \nabla_{\tilde{A}} \varphi\right)\right|^{2} \leqslant C \log \frac{1}{\varepsilon} .
$$

In view of (2. 40), we get, by classical estimates on the Riesz potential

$$
\int_{\mathbb{R}^{3}}\left|\nabla^{2} A_{\varepsilon}\right|^{2} \leqslant C \int_{\mathbb{R}^{3}} J_{\varepsilon}^{2} \leqslant C \log \frac{1}{\varepsilon} .
$$

This proves $(2.36)$. 
From (2. 36) we deduce $\left\|h_{\varepsilon}\right\|_{W^{1,2}\left(B_{1}(x)\right)}^{2} \leqslant C \log \frac{1}{\varepsilon}$ for any $x \in \mathbb{R}^{3}$. Thus by Sobolev injection we have

$$
\left(\int_{B_{1}(x)} h_{\varepsilon}^{6}\right)^{1 / 6} \leqslant C\left(\log \frac{1}{\varepsilon}\right)^{1 / 2} .
$$

Hölder's inequality on $B_{r}(x)$ leads to

$$
\int_{B_{r}(x)} h^{2} \leqslant\left|B_{r}(x)\right|^{\frac{2}{3}}\left(\int_{B_{r}(x)} h_{\varepsilon}^{6}\right)^{1 / 3} \leqslant C r^{2} \log \frac{1}{\varepsilon} .
$$

This is (2. 37). Since $\int_{B_{r}(x)}\left|\nabla h_{\varepsilon}\right|^{2}+\frac{1}{r^{2}} \int_{B_{r}(x)}\left|h_{\varepsilon}^{2}\right| \leqslant C \log \frac{1}{\varepsilon}$, taking the trace of $h$ on $\partial B_{r}(x)$ we get (2.38).

Finally (2. 39) is a consequence of (2. 35), (2. 36), which both imply $\left\|A_{\varepsilon}\right\|_{W^{2,2}\left(B_{1}(x)\right)} \leqslant C\left(\log \frac{1}{\varepsilon}\right)^{1 / 2}$ and Sobolev injections.

The last preliminary estimate is the $L^{\infty}$ bound (in $\frac{1}{\varepsilon}$ ) of $\left|\nabla_{A_{\varepsilon}} \varphi_{\varepsilon}\right|$. This estimate with (2. 36) and also (2. 32) are the only preliminary estimates which are optimal. We have the following Lemma

Lemma 2.4. There exists $C$ independent of $\varepsilon$ such that for any $x$ verifying dist $\left(x,\left\{x_{1}, \ldots, x_{n}\right\}\right) \geqslant C \varepsilon$ we have

$$
\left|\nabla_{\tilde{A}_{\varepsilon}} \varphi_{\varepsilon}\right|(x) \leqslant \frac{C}{\varepsilon}
$$

\section{Proof of Lemma 2.4.}

Let $x \in \mathbb{R}^{3} \backslash U_{i=1}^{n} B\left(x_{i}, 2 \varepsilon\right)$.

There exists $\beta>0$ depending only on $r$, the diameter of the cones $C_{i}(r)(i \leqslant$ $k$ ), and $l \leqslant k$ such that $B(x, \beta \varepsilon) \subset U_{l} \cap \mathbb{R}^{3} \backslash U_{i=1}^{n} B\left(x_{i}, \varepsilon\right)$. We will only work in $B(x, \beta \varepsilon)$. Take the Coulomb gauge in $U_{l}$ given by (2. 8$)$ and change a little bit the trivialisation on $B(x, \beta \varepsilon)$ such that the expression of $\tilde{A}$ over $B(x, \beta \varepsilon)$ becomes

$$
\forall y \in B(x, \beta \varepsilon) \quad \tilde{A}_{l}(y)=A_{l}^{0}(y)+A(y)-A(x) .
$$

We just have to multiply all the fibers over $B(x, \beta \varepsilon)$ by $e^{-i A(x) y}$. Note that (2. 2) implies

$$
\forall y \in B(x, \beta \varepsilon) \quad\left|A_{l}^{0}\right|(y) \leqslant \frac{C}{\varepsilon} \quad
$$

Combining (2. 39), (2. 42) and (2. 43) we have

$$
\forall y \in B(x, \beta \varepsilon) \quad\left|\tilde{A}_{l}\right|(y) \leqslant \frac{C}{\varepsilon} .
$$

$\varphi_{l}$ verifies the following equation in $B(x, \beta \varepsilon)$

$$
-\Delta \varphi_{l}=\frac{1}{\varepsilon^{2}} \varphi_{l}\left(1-\left|\varphi_{l}\right|^{2}\right)-\tilde{A}_{l}^{2} \varphi_{l}-2 i \tilde{A}_{l} \cdot \nabla \varphi_{l}
$$


Consider a dilation of rate $\varepsilon$ and denote

$$
\bar{\varphi}_{l}(y)=\varphi_{l}(\varepsilon y) \quad \text { and } \quad \bar{A}_{l}(y)=\varepsilon \tilde{A}_{l}(\varepsilon y) .
$$

We have

$$
-\Delta \bar{\varphi}_{l}=\bar{\varphi}_{l}\left(1-\left|\bar{\varphi}_{l}\right|^{2}\right)-\bar{A}_{l}^{2} \bar{\varphi}_{l}-2 i \bar{A}_{l} \cdot \nabla \bar{\varphi}_{l} \quad \text { in } B(x, \beta) \text {. }
$$

Let $\bar{f}_{l}=\bar{\varphi}_{l}\left(1-\left|\bar{\varphi}_{l}\right|^{2}\right)-\bar{A}_{l}^{2} \bar{\varphi}_{l}$, from (2. 44) we have

$$
\forall y \in B(x, \beta) \quad\left|\bar{f}_{l}(y)\right| \leqslant C \quad .
$$

Since $d^{*} \bar{A}_{l}=0$ on $B(x, \beta)$, we write $i \bar{A}_{l} \cdot \nabla \varphi_{l}$ in the following form

$$
2 i \bar{A}_{l} . \nabla \bar{\varphi}_{l}=d^{*}\left(2 i \bar{\varphi}_{l} \bar{A}_{l}\right)=\sum_{k=1}^{3} \frac{\partial}{\partial k}\left(i \bar{\varphi}_{l} \bar{A}_{l}^{k}\right) \text {. }
$$

Let $U$ and $V$ be the solutions of the following equations

$$
\begin{cases}-\Delta U=0 & \text { in } B(x, \beta) \\ U=\bar{\varphi}_{l} & \text { on } \partial B(x, \beta),\end{cases}
$$

and

$$
\left\{\begin{array}{ll}
-\Delta V=\bar{f}_{l}-2 d^{*}\left(i \bar{\varphi}_{l} \bar{A}_{l}\right) & \text { in } B(x, \beta) \\
V=0 & \text { on } \partial B(x, \beta)
\end{array} .\right.
$$

We clearly have $\bar{\varphi}_{l}=U+V$ in $B(x, \beta)$. Since $\left\|\bar{\varphi}_{l}\right\|_{L^{\infty}} \leqslant 1$, from elliptic estimates we have,

$$
|\nabla U|(y) \leqslant C \quad \text { in } B(x, \beta / 2) .
$$

Moreover, since $\varphi_{l} \bar{A}_{l}$ is bounded in $B(x, \beta), d^{*}\left(i \varphi_{l} \bar{A}_{l}\right) \in W^{-1, q}(B(x, \beta))$ for all $q>1$ and we have

$$
\forall 1<p<+\infty \quad \int_{B(x, \beta)}|\nabla V|^{p} \leqslant C_{p} .
$$

Combining (2. 49) and (2.50) we get

$$
\forall 1<p<+\infty \int_{B(x, \beta / 2)}|\nabla \bar{\varphi}|^{p}<C_{p} .
$$

We consider now $U_{1}$ and $V_{1}$ the solutions of the following equations

$$
\begin{cases}-\Delta U_{1}=0 & \text { in } B(x, \beta / 2) \\ U_{1}=\bar{\varphi}_{l} & \text { on } \partial B(x, \beta / 2),\end{cases}
$$

and

$$
\left\{\begin{array}{ll}
-\Delta V_{1}=\bar{f}_{l}-2 i \bar{A}_{l} \nabla \bar{\varphi}_{l} & \text { in } B(x, \beta / 2) \\
V_{1}=0 & \text { on } \partial B(x, \beta / 2)
\end{array} .\right.
$$

Clearly we have $\bar{\varphi}_{l}=U_{1}+V_{1}$ and since the right hand side of (2. 53) is bounded in $L^{p} \quad \forall p<+\infty$ we get

$$
\forall 1<p<+\infty \quad\left\|\bar{\varphi}_{l}\right\|_{W^{2, p}(B(x, \beta / 4))} \leqslant C_{p} \quad,
$$

and this leads $\left|\nabla \bar{\varphi}_{l}(x)\right|<C$ by Sobolev embedings. 
Thus we have

$$
\left|\nabla \bar{\varphi}_{l}(x)-i \bar{A}_{l} \bar{\varphi}_{l}(x)\right|<C \quad \text { indep. of } \varepsilon \quad,
$$

which is the desired result.

\subsection{The Maxwell Stress-Energy Tensor and the almost MONOTONICITY FORMULA}

Because of the renormalisation, by substracting $h_{0}^{2}$ in the energy, the conservation law of the Maxwell stress tensor given in [10] (formula (2.1), chapter 2.2 ) is modified by a term depending on $h_{0}$ which could be bad for us when we are close to the $x_{i}$ (that could make the monotonicity formula blow-up). That is the reason why we give explicitly the computation of this conservation law.

Consider $x_{0} \in \mathbb{R}^{3} \backslash\left\{x_{1}, \ldots, x_{n}\right\}$. There exists $\rho>0$ and $l \leqslant k+1$ such that $B_{\rho}\left(x_{0}\right) \subset U_{l}$.

Let $(\varphi, \tilde{A})$ be a minimizer of $G_{\varepsilon}$ (we omit here the subscript). Consider $\xi \in C_{0}^{\infty}\left(B_{\rho}\left(x_{0}\right), \mathbb{R}^{3}\right)$ and the following perturbation $\left(\varphi_{t}, \tilde{A}_{t}\right) \in V$ of $(\varphi, \tilde{A})$

$$
\forall i \leqslant k+1 \quad \begin{cases}\varphi_{i}^{t}=\varphi_{i} & \text { in } U_{i} \cap \mathbb{R}^{3} \backslash B_{\rho}\left(x_{0}\right) \\ \tilde{A}_{i}^{t}=\tilde{A}_{i} & \text { in } U_{i} \cap \mathbb{R}^{3} \backslash B_{\rho}\left(x_{0}\right)\end{cases}
$$

and

$$
\left\{\begin{array}{l}
\varphi_{l}^{t}(x)=\varphi_{l}(x+t \xi) \quad \text { in } B_{\rho}\left(x_{0}\right) \\
A_{l}^{t}(x)=A_{l}(x+t \xi) \quad \text { in } B_{\rho}\left(x_{0}\right) .
\end{array}\right.
$$

We do not write the index $l$ anymore since all the perturbation is in $U_{l}$.

We have

$$
\nabla_{\tilde{A}^{t}} \varphi^{t}(x)=\nabla_{\tilde{A}} \varphi(x+t \xi)+t \nabla \varphi(x+t \xi) . \nabla \xi(x) \quad \text { in } B_{\rho}\left(x_{0}\right)
$$

and

$$
\begin{aligned}
& h_{k l}^{t}=\tilde{h}_{k l}^{t}-h_{k l}^{0}=\frac{\partial \tilde{A}_{l}^{t}}{\partial k}-\frac{\partial \tilde{A}_{k}^{t}}{\partial l}-h_{k l}^{0} \\
= & \tilde{h}_{k l}(x+t \xi)-h_{k l}^{0}(x) \\
+ & t\left[\frac{\partial \tilde{A}_{l}}{\partial i}(x+t \xi) \frac{\partial \xi^{i}}{\partial k}-\frac{\partial \tilde{A}_{k}}{\partial i}(x+t \xi) \frac{\partial \xi^{i}}{\partial l}\right] \text { in } B_{\rho}\left(x_{0}\right) .
\end{aligned}
$$

Since we still substract $\left|h^{0}\right|^{2}$ to the energy and not $\left|h^{0, t}\right|^{2}$, we will have some change in our stress-energy tensor compared to the classical one.

Since $(\varphi, \tilde{A})$ is a minimizer we have

$$
\frac{d}{d t}\left[\int_{\mathbb{R}^{3} \backslash\left\{x_{1}, \ldots x_{n}\right\}}\left|\nabla_{\tilde{A}} \varphi^{t}\right|^{2}+\frac{1}{2 \varepsilon^{2}}\left(1-|\varphi|^{2}\right)^{2}+\frac{1}{2} \sum_{k l} h_{k l}^{t} h_{k l}^{t}\right]_{\left.\right|_{t=0}}=0
$$

Esaim : CoCv June 1996, Vol.1, PP. 77-167 
After computations we get

$$
\begin{array}{r}
\sum_{j k l} \int_{B_{\rho}\left(x_{0}\right)} \frac{\partial}{\partial j}\left[\left|\nabla_{\tilde{A}} \varphi\right|^{2}+\frac{1}{2 \varepsilon^{2}}\left(1-|\varphi|^{2}\right)^{2}+\frac{1}{2} h_{k l}\right] \xi^{j} \\
-2 \frac{\partial}{\partial k}\left(\nabla_{\tilde{A}_{k}} \varphi \frac{\partial \varphi}{\partial j}\right) \xi^{j} \\
-\left[\frac{\partial}{\partial k}\left(h_{k l} \frac{\partial \tilde{A}_{l}}{\partial j}\right)-\frac{\partial}{\partial l}\left(h_{k l} \frac{\partial \tilde{A}_{k}}{\partial j}\right)\right] \xi^{j} \\
+h_{k l} \frac{\partial}{\partial j} h_{k l}^{0} \xi^{j}=0
\end{array}
$$

(2. 56) is true for any $\xi \in C_{0}^{\infty}\left(B_{\rho}\left(x_{0}\right)\right)$, thus we have

$$
\begin{aligned}
\forall j=1,2,3 & \frac{\partial}{\partial j}\left[\left|\nabla_{\tilde{A}} \varphi\right|^{2}+\frac{1}{2 \varepsilon^{2}}\left(1-|\varphi|^{2}\right)^{2}+\frac{1}{2} \sum_{k l} h_{k l} h_{k l}\right] \\
& -2 \sum_{k} \frac{\partial}{\partial k}\left(\nabla_{\tilde{A}_{k}} \varphi, \nabla_{\tilde{A}_{j}} \varphi\right)-2 \sum_{k} \frac{\partial}{\partial k}\left(\nabla_{\tilde{A}_{k}} \varphi, i \varphi \tilde{A}_{j}\right) \\
& -\sum_{k l} \frac{\partial}{\partial k}\left(h_{k l} \tilde{h}_{j l}\right)+\sum_{k l} \frac{\partial}{\partial l}\left(h_{k l} \tilde{h}_{j k}\right) \\
& -\sum_{k l} \frac{\partial}{\partial k}\left(h_{k l} \frac{\partial \tilde{A}_{j}}{\partial l}\right)+\sum_{k l} \frac{\partial}{\partial l}\left(h_{k l} \frac{\partial \tilde{A}_{j}}{\partial k}\right) \\
& +h_{k l} \frac{\partial h_{k l}^{0}}{\partial j}=0 \quad \text { in } \mathcal{D}^{\prime}\left(B_{\rho}\left(x_{0}\right)\right) .
\end{aligned}
$$

We have

$$
\frac{\partial}{\partial k}\left[\left(\nabla_{\tilde{A}_{k}} \varphi, i \varphi\right) \tilde{A}_{j}\right]=\frac{\partial}{\partial k}\left(\nabla_{\tilde{A}_{k}} \varphi, i \varphi\right) \tilde{A}_{j}+\left(\nabla_{\tilde{A}_{k}} \varphi, i \varphi\right) \frac{\partial \tilde{A}_{j}}{\partial k}
$$

Since the second Higgs equation (2. 15) is

$$
\forall k=1,2,3 \quad \sum_{l} \frac{\partial}{\partial l} h_{l k}+\left(i \varphi, \nabla_{\tilde{A}_{k}} \varphi\right)=0
$$

and since $\sum_{k l} \frac{\partial^{2}}{\partial l \partial k} h_{l k}=0$ (because $h_{l k}=-h_{k l}$ ) we have

$$
\sum_{k} \frac{\partial}{\partial k}\left[\left(\nabla_{\tilde{A}_{k}} \varphi, i \varphi\right) \tilde{A}_{j}\right]=-\sum_{k l} \frac{\partial h_{l k}}{\partial l} \frac{\partial \tilde{A}_{j}}{\partial k}
$$

Note also that

$$
\sum_{k l}-\frac{\partial}{\partial k}\left(h_{k l} \frac{\partial \tilde{A}_{j}}{\partial l}\right)+\frac{\partial}{\partial l}\left(h_{k l} \frac{\partial \tilde{A}_{j}}{\partial k}\right)=2 \sum_{k l} \frac{\partial h_{k l}}{\partial l} \frac{\partial \tilde{A}_{j}}{\partial k}
$$


Combining (2.57), (2.57) and (2.58) we have

$$
\begin{aligned}
\forall j=1,2,3 & \frac{\partial}{\partial j}\left[\left|\nabla_{\tilde{A}} \varphi\right|^{2}+\frac{1}{2 \varepsilon^{2}}\left(1-|\varphi|^{2}\right)^{2}+\frac{1}{2} \sum_{k l} h_{k l} h_{k l}\right] \\
& -2 \sum_{k} \frac{\partial}{\partial k}\left(\nabla_{\tilde{A}_{k}} \varphi, \nabla_{\tilde{A}_{j}} \varphi\right)-2 \sum_{k l} \frac{\partial}{\partial k}\left(h_{k l} h_{j l}\right) \\
& -2 \sum_{k l} \frac{\partial}{\partial k}\left(h_{k l} h_{j l}^{0}\right)+\sum_{k l} h_{k l} \frac{\partial h_{k l}^{0}}{\partial j}=0 \text { in } \mathcal{D}^{\prime}\left(B_{\rho}\left(x_{0}\right)\right) .
\end{aligned}
$$

The previous identity is gauge invariant, thus it holds on all of $\mathbb{R}^{3} \backslash\left\{x_{1}, \ldots, x_{n}\right\}$. This is the conservation law verified by the stress tensor perturbated by the term we were looking for

$$
\sum_{k l}-2 \frac{\partial}{\partial k}\left(h_{k l} h_{k l}^{0}\right)+h_{k l} \frac{\partial h_{k l}^{0}}{\partial j}
$$

Take $x_{0} \in \mathbb{R}^{3} \backslash\left\{x_{1}, \ldots, x_{n}\right\}$ ( $x_{0}$ will be taken, in the next computation, as the centre of coordinates), for any $r<\operatorname{dist}\left(x_{0},\left\{x_{1}, \ldots, x_{n}\right\}\right)$ we multiply (2. 59) by $x_{j}$ and integrate on $B_{r}(0)$, to give

$$
\begin{aligned}
& -3 \int_{B_{r}(0)}\left|\nabla_{\tilde{A}} \varphi\right|^{2}+\frac{1}{2 \varepsilon^{2}}\left(1-|\varphi|^{2}\right)^{2}+|h|^{2} \\
& +\int_{\partial B_{r}(0)}\left(\left|\nabla_{\tilde{A}} \varphi\right|^{2}+\frac{1}{2 \varepsilon^{2}}\left(1-|\varphi|^{2}\right)^{2}+|h|^{2}\right)(x . \nu) \\
& +2 \int_{B_{r}(0)}\left|\nabla_{\tilde{A}} \varphi\right|^{2}-2 \int_{\partial B_{r}(0)} r\left(\nabla_{\tilde{A}} \varphi \cdot \nu, \nabla_{\tilde{A}}^{\varphi} \varphi \nu\right) \\
& +4 \int_{B_{r}(0)}|h|^{2}-2 r \int_{\partial B_{r}(0)} h_{k l} h_{j l} \nu^{k} \nu^{j} \\
& -2 \int_{B_{r}(0)} \sum_{j k l} x_{j} \frac{\partial}{\partial k}\left(h_{k l} h_{j l}^{0}\right)+\int_{B_{r}(0)} \sum_{j k l} x_{j} \frac{\partial}{\partial j} h_{k l}^{0} h_{k l}=0,
\end{aligned}
$$

where $\nu$ denotes the outward unit normal of $\partial B_{r}(0)$. Denote by $h \wedge \nu$ the following 1-form $h \wedge \nu_{l}=h_{k l} \nu^{k}$.

Esaim : CoCv June 1996, Vol.1, PP. 77-167 
We divide (2. 60) by $r^{2}$ and we get

$$
\begin{aligned}
& \frac{d}{d r} \quad\left[\frac{1}{r} \int_{B_{r}(0)}\left|\nabla_{\tilde{A}} \varphi\right|^{2}+\frac{3}{2 \varepsilon^{2}}\left(|\varphi|^{2}-1\right)^{2}+3|h|^{2}\right] \\
& =\frac{1}{r} \int_{\partial B_{r}(0)} 2\left|\nabla_{\tilde{A}} \varphi \cdot \nu\right|^{2}+\frac{\left(1-|\varphi|^{2}\right)^{2}}{\varepsilon^{2}}+2|h|^{2}+2|h \wedge \nu|^{2} \\
& -\quad \frac{4}{r^{2}} \int_{B_{r}(0)} h^{2}+\frac{2}{r^{2}} \int_{B_{r}(0)} \sum_{j k l} x_{j} \frac{\partial}{\partial k}\left(h_{k l} h_{j l}^{0}\right) \\
& -\frac{1}{r^{2}} \int_{B_{r}(0)} \sum_{j k l} x_{j} \frac{\partial h_{k l}^{0}}{\partial j} h_{k l} .
\end{aligned}
$$

This looks like a monotonicity formula modulo the three last integrals in the right hand side of the equality. The first one $-\frac{4}{r^{2}} \int_{B_{r}(0)} h^{2}$ is not so bad because of the estimate (2. 37). We will see that it has a very small influence. The last two look very bad since they contain derivatives on $h^{0}$ which blow-up close to the $x_{i}$. In fact, we will see that there is some compensation between the two last terms. Extracting all the quantities of (2. 61) which contain derivatives of $h_{0}$, we get

$$
\begin{aligned}
I= & \int_{B_{r}(0)} 2 \sum_{j k l} x_{j} h_{k l} \frac{\partial h_{j l}^{0}}{\partial k}-\sum_{j k l} x_{j} h_{k l} \frac{\partial h_{k l}^{0}}{\partial j} \\
= & \int_{B_{r}(0)} 2 \sum_{k<l} h_{k l}\left(\sum_{j} x_{j} \frac{\partial h_{j l}^{0}}{\partial k}-x_{j} \frac{\partial h_{j k}^{0}}{\partial l}\right) \\
& -\sum_{k<l} h_{k l}\left(\sum_{j} x_{j} \frac{\partial h_{k l}^{0}}{\partial j}-\sum_{j} x_{j} \frac{\partial h_{l k}^{0}}{\partial j}\right)
\end{aligned}
$$

which is equal to

$$
I=\int_{B_{r}(0)} 2 \sum_{k<l} h_{k l}\left(\sum_{j} x_{j}\left[\frac{\partial h_{j l}^{0}}{\partial k}+\frac{\partial h_{k j}^{0}}{\partial l}+\frac{\partial h_{l k}^{0}}{\partial j}\right]\right) .
$$


Taking $k<l$ and $j_{0}$ such that $j_{0} \neq k$ and $j_{0} \neq l$, we have

$$
\begin{aligned}
\sum_{j} x_{j}\left[\frac{\partial h_{j l}^{0}}{\partial k}+\frac{\partial h_{k j}^{0}}{\partial l}+\frac{\partial h_{l k}^{0}}{\partial j}\right] & =x_{k}\left[\frac{\partial h_{l k}^{0}}{\partial k}+\frac{\partial h_{k k}^{0}}{\partial l}+\frac{\partial h_{k l}^{0}}{\partial k}\right] \\
& +x_{l}\left[\frac{\partial h_{l l}^{0}}{\partial k}+\frac{\partial h_{l k}^{0}}{\partial l}+\frac{\partial h_{k l}^{0}}{\partial l}\right] \\
& +x_{j 0}\left[\frac{\partial h_{j_{0} l}^{0}}{\partial k}+\frac{\partial h_{k j_{0}}^{0}}{\partial l}+\frac{\partial h_{l k}^{0}}{\partial j_{0}}\right] \\
=0+0+* d h^{0} \times x_{j_{0}}=0 & .
\end{aligned}
$$

Thus $I=0$. Note also that $\sum_{l} \frac{\partial}{\partial k} h_{k l}=-\left(i \varphi, \nabla_{\tilde{A}_{l}} \varphi\right)$ and $(2.61)$ becomes

$$
\begin{aligned}
& \frac{d}{d r}\left[\frac{1}{r} \int_{B_{r}\left(x_{0}\right)}\left|\nabla_{\tilde{A}} \varphi\right|^{2}+\frac{3}{2 \varepsilon^{2}}\left(1-|\varphi|^{2}\right)^{2}+3|h|^{2}\right] \\
& =\frac{1}{r} \int_{\partial B_{r}\left(x_{0}\right)} 2\left|\nabla_{\tilde{A}} \varphi \cdot \nu\right|^{2}+\frac{1}{\varepsilon^{2}}\left(1-|\varphi|^{2}\right)^{2}+2|h|^{2}+2|h \wedge \nu|^{2} \\
& -\frac{4}{r^{2}} \int_{B_{r}\left(x_{0}\right)} h^{2}-\frac{2}{r^{2}} \int_{B_{r}\left(x_{0}\right)} \sum_{j l} h_{j l}^{0} x^{j}\left(i \varphi, \nabla_{\tilde{A}_{l}} \varphi\right) .
\end{aligned}
$$

We will deduce from (2. 62) the following Lemma

Lemma 2.5. For $\varepsilon$ sufficiently small

$$
\begin{gathered}
\forall x_{0} \in \mathbb{R}^{3} \quad \forall r>0 \\
\frac{1}{r} \int_{B_{r}\left(x_{0}\right)}\left|\nabla_{\tilde{A}} \varphi\right|^{2}+\frac{1}{\varepsilon^{2}}\left(1-|\varphi|^{2}\right)^{2}+|h|^{2} \leqslant C \log \frac{1}{\varepsilon}
\end{gathered}
$$

where $C$ does not depend on $\varepsilon$.

REMARK 2.8. In view of Lemma 2.1 and (2. 62) Lemma 2.5 is straightforward far away from the $x_{i}$ but when we are close to them there are two difficulties. Firstly the last term in (2.62) has to be controlled and secondly (2. 62) is true only for $r<\operatorname{dist}\left(x_{0},\left\{x_{1}, \ldots, x_{n}\right\}\right)$.

\section{Proof of Lemma 2.5.}

It suffices to prove $(2.63)$ for $r$ small. Let $r_{0}>0$ be small enough such that the $B\left(x_{i}, r_{0}\right)$ are disjoint.

We only have to consider the case where $x_{0}$ belongs to a $B\left(x_{i}, r_{0}\right)$, and first we study the case $x_{0}=x_{i}$.

$x_{i}$ is taken as the center of coordinates.

Multiply the three components of $(2.59)$ by the corresponding coordinates Esaim : CoCv June 1996, Vol.1, PP. 77-167 
and sum the three terms that we obtain. We get

$$
\begin{aligned}
& \sum_{j=1}^{3} x_{j} \frac{\partial}{\partial j}\left[\left|\nabla_{\tilde{A}} \varphi\right|^{2}+\frac{1}{2 \varepsilon^{2}}\left(1-|\varphi|^{2}\right)^{2}+|h|^{2}\right] \\
& -2 x_{j} \sum_{k=1}^{3} \frac{\partial}{\partial k}\left(\nabla_{\tilde{A}_{k}} \varphi, \nabla_{\tilde{A} j} \varphi\right)-2 x_{j} \sum_{k l} \frac{\partial}{\partial k}\left(h_{k l} h_{j l}\right) \\
& -2 x_{j} \sum_{k l} \frac{\partial}{\partial k}\left(h_{k l} h_{j l}^{0}\right)+x_{j} \sum_{k l} h_{k l} \frac{\partial h_{k l}^{0}}{\partial j}=0 \quad \text { in } \mathcal{D}^{\prime}\left(B_{r_{0}}(0) \backslash\{0\}\right) .
\end{aligned}
$$

We saw that

$$
\begin{gathered}
\sum_{j=1}^{3}-2 x_{j} \sum_{k l} \frac{\partial}{\partial k}\left(h_{k l} h_{j l}^{0}\right)+x_{j} \sum_{k l} h_{k l} \frac{\partial h_{k l}^{0}}{\partial j} \\
=2 \sum_{j=1}^{3} x_{j} \sum_{l=1}^{3}\left(i \varphi, \nabla_{\tilde{A}_{l}} \varphi\right) h_{j l}^{0}
\end{gathered}
$$

and (2.64) can be written in the following form

$$
\begin{aligned}
& \sum_{j=1}^{3} \frac{\partial}{\partial j}\left[x_{j}\left[\left|\nabla_{\tilde{A}} \varphi\right|^{2}+\frac{1}{2 \varepsilon^{2}}\left(1-|\varphi|^{2}\right)^{2}+h^{2}\right]\right] \\
& -2 \sum_{j, k=1}^{3} \frac{\partial}{\partial k}\left[x_{j}\left[\left(\nabla_{\tilde{A}_{k}} \varphi, \nabla_{\tilde{A}_{j}} \varphi\right)+h_{k l} h_{j l}\right]\right] \\
& -\left[\left|\nabla_{\tilde{A}} \varphi\right|^{2}+\frac{3}{2 \varepsilon^{2}}\left(1-|\varphi|^{2}\right)^{2}-|h|^{2}\right] \\
& +2 \sum_{l, j=1}^{3} x_{j} h_{j l}^{0}\left(i \varphi, \nabla_{\tilde{A} l} \varphi\right)=0 \quad \text { in } \mathcal{D}^{\prime}\left(B_{r_{0}}(0) \backslash\{0\}\right)
\end{aligned}
$$

Remark that the left-hand side of $(2.65)$ is a distribution on all of $B_{r_{0}}(0)$, we have $L^{1}$ functions, derivatives of $L^{1}$ functions and the last term is also in $L^{1}$ because $|x| \times\left|h^{0}\right| \simeq_{0} \frac{c}{|x|}$ is in $L^{p}\left(B_{r_{0}}(0)\right)$ for $p<3$ and $\left(i \varphi, \nabla_{\tilde{A}} \varphi\right) \in$ $L^{2}\left(B_{r_{0}}(0)\right)$.

We claim that the left-hand side of (2.65) that we call $D$, whose support is included in $\{0\}$ is equal to 0 in $\mathcal{D}^{\prime}\left(B_{r_{0}}(0)\right)$. It can be written in the following form

$$
D=\sum_{j=1}^{3} \frac{\partial}{\partial j}\left[x_{j} G\right]+\sum_{j, k} \frac{\partial}{\partial k}\left[x_{j} H_{k j}\right]+F
$$

where $G, H_{k j}$ and $F$ belong to $L^{1}\left(B_{r_{0}}(0)\right)$.

Since Supp $D \subset\{0\}$ in view of $(2.66), D$ is at most a Dirac at 0 . Take any $\psi \in C_{0}^{\infty}(B(0,1))$ and for any $0<\rho<r_{0}$ denote by $\psi_{\rho}$ the function 
$\psi_{\rho}(x)=\psi\left(\frac{x}{\rho}\right)$

$$
\begin{aligned}
\left|<D, \psi_{\rho}\right\rangle \mid & =\mid-\int_{B_{\rho}(0)} \sum_{j} \frac{1}{\rho} \frac{\partial \psi}{\partial j}\left(\frac{x}{\rho}\right) x_{j} G \\
& -\int_{B_{\rho}(0)} \sum_{j k} \frac{x_{j}}{\rho} \frac{\partial \psi}{\partial k}\left(\frac{x}{\rho}\right) H_{k j}+\int_{B_{\rho}(0)} \psi_{\rho} F \mid .
\end{aligned}
$$

Since $\frac{|x|}{\rho}|\nabla \psi|\left(\frac{x}{\rho}\right)$ is uniformly bounded for $|x|<\rho$ independently of $\rho$ it is clear that $\left|\left\langle D, \psi_{\rho}\right\rangle\right| \rightarrow 0$ as $\rho \rightarrow 0$ and we get that $D=0$ in $\mathcal{D}^{\prime}\left(B_{r_{0}}(0)\right)$. Integrating $D$ on $B_{r}(0)$ for any $r<r_{0}$, we get

$$
\begin{aligned}
& \frac{d}{d r}\left[\frac{1}{r} \int_{B_{r}}\left|\nabla_{\tilde{A}} \varphi\right|^{2}+\frac{3}{2 \varepsilon^{2}}\left(|\varphi|^{2}-1\right)^{2}+3|h|^{2}\right] \\
& =\frac{1}{r} \int_{\partial B_{r}(0)} 2\left|\nabla_{\tilde{A}} \varphi \cdot \nu\right|^{2}+\frac{1}{\varepsilon^{2}}\left(|\varphi|^{2}-1\right)^{2}+2|h|^{2}+2|h \wedge \nu|^{2} \\
& -\frac{4}{r^{2}} \int_{B_{r}(0)} h^{2}-\frac{2}{r^{2}} \int_{B_{r}(0)} \sum_{l}\left(i \varphi, \nabla_{\tilde{A}_{l}} \varphi\right) \sum_{j} x_{j} h_{j l}^{0} .
\end{aligned}
$$

The part of $h^{0}$ which diverges close to the pole 0 , is in view of (2), $-\frac{1}{2} d_{i} * d\left(\frac{1}{|x|}\right)$ and since $\sum_{j} x_{j} h_{j l}^{0}=x \wedge * h^{0}$ and $x \wedge \nabla \frac{1}{|x|}=0$ we have that

$$
\left|\sum_{j=1}^{3} x_{j} h_{j l}^{0}\right| \leqslant C|x| \quad \text { in } B_{r}(0)
$$

We use the following notation, $E_{r}(x)=\int_{B_{r}(0)}\left|\nabla_{\tilde{A}} \varphi\right|^{2}+\frac{3}{2 \varepsilon^{2}}\left(|\varphi|^{2}-1\right)^{2}+3|h|^{2}$ (2. 67) implies

$$
\frac{d}{d r}\left(\frac{E_{r}}{r}\right) \geqslant-\frac{4}{r^{2}} \int_{B_{r}(0)} h^{2}-\frac{2}{r} \int_{B_{r}(0)} \sum_{l}\left(i \varphi, \nabla_{\tilde{A}_{l}} \varphi\right) \sum_{j} x_{j} h_{j l}^{0}
$$

From (2. 37) and (2.68) we have

$$
\forall r \leqslant r_{0}\left|\frac{4}{r^{2}} \int_{B_{r}(0)} h^{2}+\frac{2}{r^{2}} \int_{B_{r}(0)} \sum_{l}\left(i \varphi, \nabla_{\tilde{A} l} \varphi\right) \sum_{j} x_{j} h_{j l}^{0}\right| \leqslant C \log \frac{1}{\varepsilon} .
$$

Thus $\frac{d}{d r}\left(\frac{E r}{r}\right) \geqslant-C \log \frac{1}{\varepsilon}$ and from Lemma 2.1 we have $\frac{E_{r_{0}}}{r_{0}} \leqslant \frac{C}{r_{0}} \log \frac{1}{\varepsilon}$. The two previous inequalities imply the result for $x_{0}=x_{i}$.

Take now $x_{0} \in B_{r_{0}}\left(x_{i}\right) \backslash\left\{x_{i}\right\}$. Let $d_{x_{0}}=\left|x_{0}-x_{i}\right|$. We just have established above

$$
\frac{1}{2 d_{x_{0}}} \int_{B_{2 d_{x_{0}}}\left(x_{i}\right)}\left|\nabla_{\tilde{A}} \varphi\right|^{2}+\frac{1}{2 \varepsilon^{2}}\left(1-|\varphi|^{2}\right)^{2}+|h|^{2} \leqslant C \log \frac{1}{\varepsilon} .
$$


Since $B_{\frac{d_{x_{0}}}{2}}\left(x_{0}\right) \subset B_{2 d_{x_{0}}}\left(x_{i}\right)$ the previous inequality implies

$$
\frac{2}{d_{x_{0}}} \int_{B_{\frac{d_{x_{0}}}{2}}(0)}\left|\nabla_{\tilde{A}} \varphi\right|^{2}+\frac{1}{2 \varepsilon^{2}}\left(1-|\varphi|^{2}\right)+|h|^{2} \leqslant C \log \frac{1}{\varepsilon} .
$$

For $r<\frac{d_{x_{0}}}{2}$ we have

$$
\frac{d}{d r}\left[\frac{E_{r}\left(x_{0}\right)}{r}\right] \geqslant-\left|\frac{4}{r^{2}} \int_{B_{r}\left(x_{0}\right)} h^{2}\right|-\left|\frac{2}{r^{2}} \int_{B_{r}\left(x_{0}\right)}\right| \nabla_{\tilde{A}} \varphi|\times| x \wedge * h^{0}||
$$

We use the following bound $\left|x \wedge * h^{0}\right| \leqslant c \frac{|x|}{d_{x_{0}}^{2}}$ and (2.70) implies

$$
\frac{d}{d r}\left[\frac{E_{r}\left(x_{0}\right)}{r}\right] \geqslant-C \log \frac{1}{\varepsilon}-C \frac{r}{d_{x_{0}}^{2}}\left(\frac{E_{r}\left(x_{0}\right)}{r}\right)^{1 / 2},
$$

where we have used (2. 37) and Cauchy-Schwarz inequality. In particular, (2. 71) implies

$$
\frac{d}{d r}\left[\frac{E_{r}\left(x_{0}\right)}{r}+C r \log \frac{1}{\varepsilon}\right] \leqslant-\frac{C r}{d_{x_{0}}^{2}}\left[\frac{E_{r}\left(x_{0}\right)}{r}+C r \log \frac{1}{\varepsilon}\right]^{1 / 2}
$$

Integrating (2. 70) between $\frac{d_{x_{0}}}{2}$ and any $\rho<\frac{d_{x_{0}}}{2}$, the bound (2. 69) yields

$$
\forall \rho \leqslant \frac{d_{x_{0}}}{2}, \quad \frac{E_{\rho}\left(x_{0}\right)}{\rho} \geqslant C \log \frac{1}{\varepsilon},
$$

and the lemma is proved for any $x_{0} \in \cup_{i=1}^{n} B\left(x_{i}, r_{0}\right)$. On the remains of $\mathbb{R}^{3}$, as we mentioned above, the proof of $(2.63)$ is much more straightforward.

\section{THE $\eta$-COMPACTNESS LEMMA}

This part is devoted to the proof of the following lemma.

LEMma 3.1. There exists $\eta>0, \nu$ and $\lambda>1$ such that for any $\varepsilon$ sufficiently small, for any $(\varphi, \tilde{A}) \in V$ minimizer of $G_{\varepsilon}$, for any $x_{0} \in \mathbb{R}^{3} \backslash\left\{x_{1}, \ldots x_{n}\right\}$ and for any $\lambda \varepsilon \leqslant \rho \leqslant \min \left(\nu, \eta d_{x_{0}}\right)$, where $d_{x_{0}}=\operatorname{dist}\left(x_{0},\left\{x_{1}, \ldots, x_{n}\right\}\right)$, if

$$
\frac{1}{\rho} \int_{B_{\rho}\left(x_{0}\right)}\left|\nabla_{\tilde{A}} \varphi\right|^{2}+\frac{1}{2 \varepsilon^{2}}\left(1-|\varphi|^{2}\right)^{2}+|h|^{2} \leqslant \eta \log \left(\frac{\rho}{\varepsilon}\right)
$$

then

$$
|\varphi| \geqslant \frac{1}{2} \quad \text { in } \quad B_{\rho / 2}\left(x_{0}\right)
$$

\section{Proof of Lemma 3.1.}

First of all, we introduce some useful notations. Denote

$$
\begin{gathered}
E_{r}\left(x_{0}\right)=\int_{B_{r}\left(x_{0}\right)}\left|\nabla_{\tilde{A}} \varphi\right|^{2}+\frac{3}{2 \varepsilon^{2}}\left(1-|\varphi|^{2}\right)^{2}+3|h|^{2}, \\
I_{r}\left(x_{0}\right)=\int_{\partial B_{r}\left(x_{0}\right)}\left|\nabla_{\tilde{A}} \varphi\right|^{2}+\frac{3}{2 \varepsilon^{2}}\left(1-|\varphi|^{2}\right)^{2}+3|h|^{2}=\frac{d}{d r} E_{r}\left(x_{0}\right), \\
F_{r}\left(x_{0}\right)=\int_{B_{r}\left(x_{0}\right)} 2\left|\nabla_{\tilde{A}} \varphi \cdot \nu\right|^{2}+\frac{1}{\varepsilon^{2}}\left(1-|\varphi|^{2}\right)^{2}+2|h|^{2}+2|h \wedge \nu|^{2} \\
\text { Esaim: Cocv June 1996, Vol.1, pr. } 77-167
\end{gathered}
$$




$$
J_{r}\left(x_{0}\right)=\int_{\partial B_{r}\left(x_{0}\right)} 2\left|\nabla_{\tilde{A}} \varphi \cdot \nu\right|^{2}+\frac{1}{\varepsilon^{2}}\left(1-|\varphi|^{2}\right)^{2}+2|h|^{2}+2|h \wedge \nu|^{2}=\frac{d F_{r}\left(x_{0}\right)}{d r}
$$

and

$$
R_{r}\left(x_{0}\right)=-\frac{4}{r^{2}} \int_{B_{r}\left(x_{0}\right)} h^{2}-\frac{2}{r^{2}} \int_{B_{r}\left(x_{0}\right)} \sum_{j l} h_{j l}^{0} x^{j}\left(i \varphi, \nabla_{\tilde{A}_{l}} \varphi\right) .
$$

Using those notations (2.62) becomes

$$
\frac{d}{d r}\left[\frac{E_{r}\left(x_{0}\right)}{r}\right]=\frac{J_{r}\left(x_{0}\right)}{r}+R_{r}\left(x_{0}\right) .
$$

As in the proof of Lemma 2.5 we use the bound

$$
\left|R_{r}\left(x_{0}\right)\right| \leqslant C \log \frac{1}{\varepsilon}+C \frac{r}{d_{x_{0}}^{2}}\left(\frac{E_{r}\left(x_{0}\right)}{r}\right)^{1 / 2} .
$$

Suppose for some $\lambda \epsilon<\rho<\frac{d_{x_{0}}}{2}$ we have $E_{\rho} / \rho \leqslant \eta \log (\rho / \varepsilon)(\lambda>1$ will be fixed later but we already consider couples $(\eta, \lambda)$ such that $\log \lambda>\frac{1}{\eta}$. As in the proof of Lemma 2.5, using (3. 1) and (3. 2), we prove that $\forall r<\rho \quad \frac{E_{r}\left(x_{0}\right)}{r} \leqslant C \eta \log \left(\frac{\rho}{\varepsilon}\right)$ and combined with (3. 2) this gives

$$
\forall r<\rho \quad\left|R_{r}\left(x_{0}\right)\right| \leqslant C \log \frac{1}{\varepsilon}+C \frac{r}{d_{x_{0}}^{2}}\left(\eta \log \left(\frac{\rho}{\varepsilon}\right)\right)^{1 / 2} .
$$

Integrating (3. 2) between $\rho$ and $\lambda \varepsilon$ ( $\lambda$ will be fixed later) we have

$$
\int_{\lambda \varepsilon}^{\rho} \frac{J_{r}\left(x_{0}\right)}{r} d r \leqslant \eta \log \left(\frac{\eta}{\varepsilon}\right)+C \rho \log \frac{1}{\varepsilon}+C \frac{\rho^{2}}{d_{x_{0}}^{2}}\left(\eta \log \left(\frac{\rho}{\varepsilon}\right)\right)^{1 / 2} .
$$

It is clear that there exists $\nu>0$ independent of $\varepsilon$ for $\varepsilon$ small enough such that, if $\rho<\nu$ and $\rho<\frac{d_{x_{0}}}{2}$, we have

$$
C \rho \log \frac{1}{\varepsilon}+C \frac{\rho^{2}}{d_{x_{0}}^{2}}\left(\eta \log \left(\frac{\rho}{\varepsilon}\right)\right)^{1 / 2} \leqslant \eta \log \left(\frac{\rho}{\varepsilon}\right)
$$

( $\lambda$ has also to be chosen sufficiently large (independently of $\varepsilon$ ) such that $\left.c(\eta \log \lambda)^{1 / 2} \leqslant \eta \log \lambda\right)$.

Finally, for $\lambda \varepsilon \leqslant \rho \leqslant \min \left[d_{x_{0}} / 2, \nu\right]$, we have

$$
\int_{\lambda \varepsilon}^{\rho} \frac{J_{r}\left(x_{0}\right)}{r} d r \leqslant 2 \eta \log \left(\frac{\rho}{\varepsilon}\right)
$$

Thus we have also

$$
\int_{\lambda \varepsilon}^{\rho} \frac{J_{r}\left(x_{0}\right)}{r}+\frac{J_{r / 2}\left(x_{0}\right)}{r} d r \leqslant C \eta \log \left(\frac{\rho}{\varepsilon}\right) .
$$

Moreover

$$
\begin{aligned}
\int_{\lambda \varepsilon}^{\rho} \frac{J_{r}\left(x_{0}\right)}{r}=\int_{\lambda \varepsilon}^{\rho} \frac{1}{r} \frac{d}{d r} F_{r}\left(x_{0}\right) & =\left[\frac{F_{\rho}}{\rho}\left(x_{0}\right)-\frac{F_{\lambda \varepsilon}\left(x_{0}\right)}{\lambda \varepsilon}\right]+ \\
& +\int_{\lambda \varepsilon}^{\rho} \frac{F_{r}\left(x_{0}\right)}{r^{2}} d r
\end{aligned}
$$


and this implies

$$
\frac{F_{\lambda \varepsilon}\left(x_{0}\right)}{\lambda \varepsilon}+C \eta \log \left(\frac{\rho}{\varepsilon}\right) \geqslant \int_{\lambda \varepsilon}^{\rho} \frac{F_{r}\left(x_{0}\right)}{r^{2}} d r,
$$

but $F_{\lambda \varepsilon}\left(x_{0}\right) / \lambda \varepsilon \leqslant E_{\lambda \varepsilon}\left(x_{0}\right) / \lambda \varepsilon \leqslant C \eta \log \left(\frac{\rho}{\varepsilon}\right)$, and this yields

$$
\int_{\lambda \varepsilon}^{\rho} \frac{J_{r}\left(x_{0}\right)}{r}+\frac{J_{r / 2}\left(x_{0}\right)}{r}+\frac{F_{r}\left(x_{0}\right)}{r^{2}} d r \leqslant C \eta \log \left(\frac{\rho}{\varepsilon}\right) .
$$

Applying the mean value formula in (3. 6) we get the existence of $r_{0} \in[\lambda \varepsilon, \rho]$ such that

$$
J_{r_{0}}\left(x_{0}\right)+J_{r_{0 / 2}}\left(x_{0}\right)+\frac{F_{r_{0}}}{r_{0}}\left(x_{0}\right) \leqslant C \eta
$$

where $C$ is independent on $\varepsilon$ and $\eta$. Our aim is to prove that (3. 7) implies that

$$
\frac{E_{r_{0 / 2}}\left(x_{0}\right)}{r_{0 / 2}} \leqslant f(\eta) \text { where } f(\eta) \rightarrow 0 \text { as } \eta \rightarrow 0 .
$$

And (3. 8) will imply (see below at the end of the proof of the Lemma),

$$
\begin{aligned}
\frac{E_{\varepsilon}\left(x_{0}\right)}{\varepsilon} & \leqslant f(\eta)+\int_{\varepsilon}^{r_{0} / 2}\left|R_{r}\left(x_{0}\right)\right| \\
& \leqslant g(\eta) \quad \text { with } g \rightarrow 0 \text { as } \eta \rightarrow 0 .
\end{aligned}
$$

This yields in particular

$$
\frac{1}{\left|B_{\varepsilon}\left(x_{0}\right)\right|} \int_{B_{\varepsilon}\left(x_{0}\right)}\left(1-|\varphi|^{2}\right)^{2} \leqslant g(\eta)
$$

and since $\left.|\nabla| \varphi\right|^{2}|\leqslant| \nabla|\varphi||\leqslant| \nabla_{\tilde{A}} \varphi \mid \leqslant C / \varepsilon$, for $g(\eta)$ sufficiently small (3. 10) implies $|\varphi| \geqslant \frac{1}{2}$ in $B_{\lambda \varepsilon}\left(x_{0}\right)$ and in particular $|\varphi|\left(x_{0}\right) \geqslant \frac{1}{2}$, which is the desired result.

The difficulty now is to obtain (3. 8) from (3. 7). (3. 7) says that the total energy is small except possibly the tangential projections of $\nabla_{\tilde{A}} \varphi$ on all the spheres in $B_{r_{0}}\left(x_{0}\right)$ of center $x_{0}$.

First of all note that we can take $r_{0} \in\left[\lambda \varepsilon, \frac{\eta}{\operatorname{Ln} \frac{1}{\varepsilon}}\right]$. This is clear if $\rho<\frac{\eta}{\log \frac{1}{\varepsilon}}$, otherwise, since $\rho$ verifies

$$
\frac{E_{\rho}\left(x_{0}\right)}{\rho} \leqslant \eta \log \left(\frac{\rho}{\varepsilon}\right)
$$

as we mentioned above we have

$$
\forall r<\rho \quad \frac{E_{r}\left(x_{0}\right)}{r} \leqslant C \eta \log \left(\frac{\rho}{\varepsilon}\right) .
$$

Take $r=\frac{\eta}{\log \frac{1}{\varepsilon}}=r^{\prime}$ and we have

$$
\begin{aligned}
& \frac{E_{r^{\prime}}\left(x_{0}\right)}{r^{\prime}} \leqslant C \eta \log \left(\frac{r^{\prime}}{\varepsilon}\right)+C \eta \log \left(\frac{\rho}{r^{\prime}}\right) \\
& \leqslant C \eta \log \left(\frac{r^{\prime}}{\varepsilon}\right)+C \eta \log \left[\frac{1}{\eta} \log \frac{1}{\varepsilon}\right] \\
& \text { Esaim : COCV June 1996, Vol.1, fr. } 77-167
\end{aligned}
$$


but $\log \left[\frac{1}{\eta} \log \frac{1}{\varepsilon}\right]<\log \left(\frac{\eta}{\varepsilon \log \frac{1}{\varepsilon}}\right)$ (for $\varepsilon$ sufficiently small), thus we have $E_{r^{\prime}}\left(x_{0}\right) / r^{\prime} \leqslant C \eta \log \left(r^{\prime} / \varepsilon\right)$ and we get (3. 7) for $r_{0} \in\left[\lambda \varepsilon, \eta / \log \frac{1}{\varepsilon}\right]$.

Since $r_{0}<\eta \operatorname{dist}\left(x_{0},\left\{x_{1}, \ldots, x_{n}\right\}\right)$ we can clearly include $B_{r_{0}}\left(x_{0}\right)$ in some $U_{l}$. We trivialize $E$ over $B_{r_{0}}\left(x_{0}\right)$ and consider the particular representative of $(\varphi, \tilde{A})$ in $B_{r_{0}}\left(x_{0}\right) \subset U_{l}$ given by Proposition 2.1 modulo the multiplication of the fiber over $y$ by $e^{-i A\left(x_{0}\right) \cdot y}$ for each $y$ in $B_{r_{0}}\left(x_{0}\right)$, and we have

$$
\tilde{A}_{l}(y)=A_{l}^{0}(y)+A(y)-A\left(x_{0}\right) .
$$

Because of (2. 39) we have

$$
\begin{aligned}
\left|\tilde{A}_{l}(y)\right| & \leqslant\left|A_{l}^{0}(y)\right|+C \frac{\eta^{1 / 2}}{\left(\operatorname{Ln} \frac{1}{\varepsilon}\right)^{1 / 2}}(\log 1 / \varepsilon)^{1 / 2} \\
& \leqslant \frac{C}{d_{x_{0}}}+C \eta^{1 / 2} .
\end{aligned}
$$

We change the scale in view of working in a unit ball and multiply by $\frac{1}{r_{0}}$. Denote in $B\left(x_{0}, 1\right)$

$$
\begin{aligned}
\bar{\varphi}(x) & =\varphi\left(r_{0} x\right) \\
\hat{A}(x)=r_{0} \tilde{A}\left(r_{0} x\right) & =r_{0} A_{l}^{0}\left(r_{0} x\right)+r_{0} A\left(r_{0} x\right) \\
\bar{A}(x) & =r_{0} A\left(r_{0} x\right) \\
\text { and } \quad \bar{h}(x)=d \bar{A}(x) & =r_{0}^{2} d A\left(r_{0} x\right)=r_{0}^{2} h\left(r_{0} x\right) .
\end{aligned}
$$

Thus we have, in particular,

$$
\nabla_{\hat{A}} \bar{\varphi}(x)=r_{0} \nabla_{\tilde{A}} \varphi\left(r_{0} x\right)
$$

and

$$
d^{*} \bar{h}(x)=r_{0}^{3} d^{*} h\left(r_{0} x\right)
$$

moreover $(\bar{\varphi}, \hat{A})$ minimizes the functional

$$
\bar{G}_{\varepsilon, r_{0}}(\bar{\varphi}, \hat{A})=\int_{B_{1}}\left|\nabla_{\hat{A}} \bar{\varphi}\right|^{2}(x)+\frac{1}{2}\left(\frac{r_{0}}{\varepsilon}\right)^{2}\left(1-|\bar{\varphi}|^{2}\right)^{2}(x)+\frac{1}{r_{0}^{2}}|\bar{h}|^{2}(x) d x
$$

and verifies the equations

$$
\left\{\begin{aligned}
\nabla_{\hat{A}}^{2} \bar{\varphi} & =\left(\frac{r_{0}}{\varepsilon}\right)^{2} \bar{\varphi}\left(1-|\bar{\varphi}|^{2}\right) \\
-\frac{d^{*} \bar{h}}{r_{0}^{2}} & =\left(i \bar{\varphi}, \nabla_{\hat{A}} \bar{\varphi}\right) .
\end{aligned}\right.
$$

(3. 12) implies, in particular,

$$
\left(i \bar{\varphi}, \nabla_{\hat{A}}^{2} \bar{\varphi}\right)(x)=0
$$

where

$$
\begin{aligned}
\nabla_{\hat{A}}^{2} \bar{\varphi} & =\sum_{k=1}^{3}\left(\frac{\partial}{\partial k}-i \hat{A}_{k}\right)\left(\frac{\partial \bar{\varphi}}{\partial k}-i \hat{A}_{k} \bar{\varphi}\right) \\
& =\Delta \bar{\varphi}-2 i \hat{A} \cdot \nabla \bar{\varphi}-i d^{*} \hat{A} \bar{\varphi}-|\bar{A}|^{2} \bar{\varphi} .
\end{aligned}
$$


But we have chosen a gauge such that $d^{*} \hat{A}=0$ and (3. 12) becomes

$$
(i \bar{\varphi}, \Delta \bar{\varphi})=\hat{A} \cdot \nabla|\bar{\varphi}|^{2}
$$

For $\sigma \in\left[\frac{1}{2}, 1\right]$, denote by $\Delta_{\sigma}$ the Laplace Beltrami operator on $\partial B_{\sigma} \subset \mathbb{R}^{3}$ and by $d_{\top}$ and $(*)$ we denote, respectively, the exterior derivative and the Hodge operator on $\partial B_{\sigma} \subset \mathbb{R}^{3}$. By definition we have

$$
\Delta_{\sigma}=d_{\top}(*) d_{\top}(*)+(*) d_{\top}(*) d_{\top} \quad \text { on } \partial B_{\sigma} \quad .
$$

If $f$ is a 0 -form in $B_{1}$, for $\sigma \in\left[\frac{1}{2}, 1\right]$ we have

$$
\Delta f=\Delta_{\sigma} f+\frac{\partial^{2} f}{\partial r^{2}}+\frac{2}{r} \frac{\partial f}{\partial r}
$$

In particular we have

$$
\begin{aligned}
\Delta\left(i \bar{\varphi}, \frac{\partial \bar{\varphi}}{\partial \sigma}\right)=\Delta_{\sigma}\left(i \bar{\varphi}, \frac{\partial \bar{\varphi}}{\partial \sigma}\right) & +\frac{\partial^{2}}{\partial \sigma^{2}}\left(i \bar{\varphi}, \frac{\partial \bar{\varphi}}{\partial \sigma}\right) \\
& +\frac{2}{\sigma} \frac{\partial}{\partial \sigma}\left(i \bar{\varphi}, \frac{\partial \bar{\varphi}}{\partial \sigma}\right)
\end{aligned}
$$

Using Lemma A.3 and (3. 14) we have,

$$
\begin{aligned}
\Delta\left(i \bar{\varphi}, \frac{\partial \bar{\varphi}}{\partial \sigma}\right) & =\frac{\partial}{\partial \sigma}\left(i \bar{\varphi}, \Delta_{\sigma} \bar{\varphi}\right)+2(*) d_{\top}\left[\left(\frac{\partial \bar{\varphi}}{\partial \sigma}, i(*) d_{\top} \bar{\varphi}\right)\right] \\
& +\frac{2}{\sigma}\left(i \bar{\varphi}, \Delta_{\sigma} \bar{\varphi}\right)+\frac{\partial^{2}}{\partial \sigma^{2}}\left(i \bar{\varphi}, \frac{\partial \bar{\varphi}}{\partial \sigma}\right) \\
& +\frac{2}{\sigma} \frac{\partial}{\partial \sigma}\left(i \bar{\varphi}, \frac{\partial \bar{\varphi}}{\partial \sigma}\right) .
\end{aligned}
$$

Using the Laplace Beltrami operator (3. 13) is equivalent to

$$
\left(i \bar{\varphi}, \Delta_{\sigma} \bar{\varphi}\right)=\hat{A} . \nabla|\bar{\varphi}|^{2}-\frac{\partial}{\partial \sigma}\left(i \bar{\varphi}, \frac{\partial \bar{\varphi}}{\partial \sigma}\right)-\frac{2}{\sigma}\left(i \bar{\varphi}, \frac{\partial \bar{\varphi}}{\partial \sigma}\right)
$$

Combining (3.15) and (3.16) we get

$$
\begin{aligned}
& \Delta\left(i \bar{\varphi}, \frac{\partial \bar{\varphi}}{\partial \sigma}\right)=2(*) d_{\top}\left[\left(\frac{\partial \bar{\varphi}}{\partial \sigma}, i(*) d_{\top} \bar{\varphi}\right)\right]+\frac{\partial}{\partial \sigma}\left(i \bar{\varphi}, \frac{\partial \bar{\varphi}}{\partial \sigma}\right) \\
&-\frac{\partial^{2}}{\partial \sigma^{2}}\left(i \bar{\varphi}, \frac{\partial \bar{\varphi}}{\partial \sigma}\right)+\frac{2}{\sigma^{2}}\left(i \bar{\varphi}, \frac{\partial \bar{\varphi}}{\partial \sigma}\right)-\frac{2}{\sigma} \frac{\partial}{\partial \sigma}\left(i \bar{\varphi}, \frac{\partial \bar{\varphi}}{\partial \sigma}\right) \\
&+\frac{2}{\sigma}\left(\hat{A} . \nabla|\bar{\varphi}|^{2}\right)-\frac{2}{\sigma} \frac{\partial}{\partial \sigma}\left(i \bar{\varphi}, \frac{\partial \bar{\varphi}}{\partial \sigma}\right)-\frac{4}{\sigma^{2}}\left(i \bar{\varphi}, \frac{\partial \bar{\varphi}}{\partial \sigma}\right) \\
&+\frac{\partial^{2}}{\partial \sigma^{2}}\left(i \bar{\varphi}, \frac{\partial \bar{\varphi}}{\partial \sigma}\right)+\frac{2}{\sigma} \frac{\partial}{\partial \sigma}\left(i \bar{\varphi}, \frac{\partial \bar{\varphi}}{\partial \sigma}\right) \\
& \text { Esaim: COCV June 1996, Vol.1, Pr. 77-167 }
\end{aligned}
$$


The cancelation of the two maximal order terms $\frac{\partial^{2}}{\partial \sigma^{2}}\left(i \bar{\varphi}, \frac{\partial \bar{\varphi}}{\partial \sigma}\right)$, in the right hand side of (3. 17), is a crucial point of the proof. We have

$$
\begin{aligned}
\Delta\left(i \bar{\varphi}, \frac{\partial \bar{\varphi}}{\partial \sigma}\right) & =2(*) d_{\top}\left[\left(\frac{\partial \bar{\varphi}}{\partial \sigma}, i(*) d_{\top \bar{\varphi}}\right)\right]-\frac{2}{\sigma^{2}} \frac{\partial}{\partial \sigma}\left[\sigma\left(i \bar{\varphi}, \frac{\partial \bar{\varphi}}{\partial \sigma}\right)\right] \\
& +\frac{\partial}{\partial \sigma}\left(\hat{A} . \nabla|\bar{\varphi}|^{2}\right)+\frac{2}{\sigma} \hat{A} . \nabla|\bar{\varphi}|^{2}
\end{aligned}
$$

Denote by $T_{1}$ the following set $T_{1}=B\left(x_{0}, 1\right) \backslash B\left(x_{0}, 1 / 2\right)$. Let $V$ be the 1-form solution of

$$
\begin{cases}\Delta V=\left(\frac{\partial \bar{\varphi}}{\partial \sigma}, i(*) d_{\top} \bar{\varphi}\right) & \text { in } T_{1} \\ V=0 & \text { on } \partial T_{1} .\end{cases}
$$

By classical elliptic estimates we have $\forall q<3 / 2$

$$
\begin{aligned}
\left(\int_{T_{1}}|\nabla V|^{q}\right)^{1 / q} & \leqslant C_{q}\left\|\left(\frac{\partial \bar{\varphi}}{\partial \sigma}, i(*) d_{\top} \bar{\varphi}\right)\right\|_{L^{1}} \\
& \leqslant C_{q}\left(\int_{T_{1}}\left|\frac{\partial \bar{\varphi}}{\partial \sigma}\right|^{2}\right)^{1 / 2}\left(\int_{T_{1}}\left|d_{\top} \bar{\varphi}\right|^{2}\right)^{1 / 2} .
\end{aligned}
$$

Moreover

$$
d_{\top} \Delta V=d_{\top}\left(\frac{\partial \bar{\varphi}}{\partial \sigma}, i(*) d_{\top} \bar{\varphi}\right)
$$

thus

$$
(*) d_{\top}\left(\frac{\partial \bar{\varphi}}{\partial \sigma}, i(*) d_{\top} \bar{\varphi}\right)=<d_{\top} \Delta V, \omega>
$$

where $\omega=* d \sigma$. Applying Lemma A.4 we have

$$
\begin{aligned}
\left\langle d_{\top} \Delta V, \omega\right\rangle & \left.\left.=<\Delta d_{\top} V, \omega\right\rangle+\frac{4}{\sigma^{2}} \frac{\partial}{\partial \sigma}[\sigma<d V, \omega\rangle\right] \\
& -\frac{2}{\sigma} \frac{\partial}{\partial \sigma}\langle d V, \omega\rangle \\
& \left.=<\Delta[\langle d V, \omega\rangle \omega], \omega\rangle+\frac{4}{\sigma^{2}} \frac{\partial}{\partial \sigma}[\sigma<d V, \omega\rangle\right] \\
& -\frac{2}{\sigma} \frac{\partial}{\partial \sigma}\langle d V, \omega\rangle .
\end{aligned}
$$


Applying the first formula of Lemma A.4 we have

$$
\begin{aligned}
\left\langle d_{\top} \Delta V, \omega\right\rangle & =\Delta<d V, \omega\rangle \\
& \left.-\frac{2}{\sigma^{2}}\langle d V, \omega\rangle+\frac{4}{\sigma^{2}} \frac{\partial}{\partial \sigma}[\sigma<d V, \omega\rangle\right] \\
& -\frac{2}{\sigma} \frac{\partial}{\partial \sigma}\langle d V, \omega\rangle \\
& =\Delta<d V, \omega\rangle+\frac{2}{\sigma^{2}} \frac{\partial}{\partial \sigma}[\sigma\langle d V, \omega\rangle] .
\end{aligned}
$$

Combining (3. 21) and (3. 22) we get

$$
\Delta<d V, \omega\rangle=(*) d_{\top}\left(\frac{\partial \bar{\varphi}}{\partial \sigma}, i(*) d_{\top} \bar{\varphi}\right)-\frac{2}{\sigma^{2}} \frac{\partial}{\partial \sigma}[\sigma<d V, \omega>]
$$

Subtracting (3. 23) and (3. 18)

$$
\begin{aligned}
\Delta \quad & {\left[\left(i \bar{\varphi}, \frac{\partial \bar{\varphi}}{\partial \sigma}\right)-2\langle d V, \omega\rangle\right]=-\frac{2}{\sigma^{2}} \frac{\partial}{\partial \sigma}\left[\left(i \bar{\varphi}, \frac{\partial \bar{\varphi}}{\partial \sigma}\right)\right] } \\
+ & \frac{\partial}{\partial \sigma}\left(\hat{A} . \nabla|\bar{\varphi}|^{2}\right)+\frac{2}{\sigma} \hat{A} . \nabla|\varphi|^{2}+\frac{4}{\sigma^{2}} \frac{\partial}{\partial \sigma}[\sigma\langle d V, \omega\rangle] .
\end{aligned}
$$

Let $H_{1}$ be the solution of

$$
\left\{\begin{aligned}
\Delta H_{1}= & -\frac{2}{\sigma^{2}} \frac{\partial}{\partial \sigma}\left[\sigma\left(i \bar{\varphi}, \frac{\partial \bar{\varphi}}{\partial \sigma}\right)\right]+\frac{\partial}{\partial \sigma}\left(\hat{A} \cdot \nabla|\bar{\varphi}|^{2}\right) \\
& \left.+\frac{2}{\sigma} \hat{A} \cdot \nabla|\bar{\varphi}|^{2}+\frac{4}{\sigma^{2}} \frac{\partial}{\partial \sigma}[\sigma<d V, \omega\rangle\right] \\
H_{1}=0 & \text { on } \partial T_{1} .
\end{aligned}\right.
$$

Remark that, since $d^{*} \hat{A}=0$, we have $\hat{A} \cdot \nabla|\bar{\varphi}|^{2}=d^{*}\left(\hat{A}|\bar{\varphi}|^{2}\right)$ and

$$
\frac{\partial}{\partial \sigma} d^{*}\left(\hat{A}|\bar{\varphi}|^{2}\right)-d^{*}\left(\frac{\partial}{\partial \sigma}\left(\hat{A}|\bar{\varphi}|^{2}\right)\right)=-\frac{1}{\sigma} d^{*}\left(\hat{A}|\bar{\varphi}|^{2}\right)+\frac{1}{\sigma} \frac{\partial}{\partial \sigma}\left(\hat{A} \cdot \nu|\bar{\varphi}|^{2}\right)
$$

Thus

$$
\left\{\begin{array}{l}
\Delta H_{1}=-\frac{2}{\sigma^{2}} \frac{\partial}{\partial \sigma}\left[\sigma\left[\left(i \bar{\varphi}, \frac{\partial \bar{\varphi}}{\partial \sigma}\right)-2<d V, \omega>\right]\right]+ \\
\quad+d^{*}\left(\frac{\partial}{\partial \sigma}\left(\hat{A}|\bar{\varphi}|^{2}\right)\right)+\frac{1}{\sigma} d^{*}\left(\hat{A} \cdot \nu|\bar{\varphi}|^{2}\right)+\frac{1}{\sigma} \frac{\partial}{\partial \sigma}\left(\hat{A} \cdot \nu|\bar{\varphi}|^{2}\right) \text { in } T_{1} \\
H_{1}=0 \quad \text { on } \partial T_{1}
\end{array}\right.
$$


By classical elliptic estimates we have $\forall p<\frac{3}{2}$

$$
\begin{aligned}
\left(\int_{T_{1}}\left|\nabla H_{1}\right|^{p}\right)^{1 / p} & \leqslant C_{p}\left[\left(\int_{T_{1}}\left|\frac{\partial \bar{\varphi}}{\partial \sigma}\right|^{p}\right)^{1 / p}+\left(\int_{T_{1}}|\nabla v|^{p}\right)^{1 / p}\right. \\
& \left.+\left(\int_{T_{1}}\left|\frac{\partial}{\partial \sigma}\left(\hat{A}|\bar{\varphi}|^{2}\right)\right|^{p}\right)^{1 / p}+\left(\left.\left.\int_{T_{1}}|\hat{A}| \bar{\varphi}\right|^{2}\right|^{p}\right)^{1 / p}\right]
\end{aligned}
$$

Let $H_{2}$ be the solution of

$$
\left\{\begin{array}{l}
\Delta H_{2}=0 \text { in } T_{1} \\
\left.H_{2}=\left(i \bar{\varphi}, \frac{\partial \bar{\varphi}}{\partial \sigma}\right)-2<d V, \omega\right\rangle \\
=\left(i \bar{\varphi}, \frac{\partial \bar{\varphi}}{\partial \sigma}\right)-2(*) d_{\top} V=\left(i \bar{\varphi}, \frac{\partial \bar{\varphi}}{\partial \sigma}\right) \quad \text { on } \partial T_{1} \quad .
\end{array}\right.
$$

Let $T_{1}^{\prime}$ be the following subannulus of $T_{1}, T_{1}^{\prime}=B_{7 / 8}\left(x_{0}\right) \backslash B_{5 / 8}\left(x_{0}\right)$. By classical results on harmonic functions we have

$$
\left(\int_{T_{1}^{\prime}}\left|\nabla H_{2}\right|^{2}\right)^{1 / 2} \leqslant C\left(\int_{\partial T_{1}}\left|\frac{\partial \bar{\varphi}}{\partial \sigma}\right|^{2}\right)^{1 / 2} .
$$

Combining (3. 24), (3. 25) and (3. 28), it is clear that

$$
\left(i \bar{\varphi}, \frac{\partial \bar{\varphi}}{\partial \sigma}\right)=2\langle d V, \omega\rangle+H_{1}+H_{2} \quad \text { in } T_{1}
$$

The conclusion at this point is that we have been able to write $\left(i \bar{\varphi}, \frac{\partial \bar{\varphi}}{\partial \sigma}\right)$ (which includes the radial derivative) using tangential derivatives: $\langle d V, \omega\rangle$ plus some remaining terms $H_{1}$ and $H_{2}$ which are not so bad (we will see this below).

For $1<p<\frac{3}{2}$ and $1<q<\frac{3}{2}$ fixed and to be made precise later, applying the mean value formula, we can find $t \in\left[\frac{5}{8}, \frac{7}{8}\right]$ such that, for $C$ sufficiently large, we have simultaneously

$$
\begin{gathered}
\left(\int_{\partial B_{t}}\left|\nabla H_{1}\right|^{p}\right)^{\frac{1}{p}} \leqslant C\left(\int_{T_{1}}\left|\nabla H_{1}\right|^{p}\right)^{1 / p}, \\
\left(\int_{\partial B_{t}}\left|\nabla H_{2}\right|^{2}\right)^{1 / 2} \leqslant C\left(\int_{T_{1}^{\prime}}\left|\nabla H_{2}\right|^{2}\right)^{1 / 2} \\
\left(\int_{\partial B_{t}}|\nabla V|^{q}\right)^{1 / q} \leqslant C\left(\int_{T_{1}}|\nabla V|^{q}\right)^{1 / q}, \\
\left(\int_{\partial B_{t}}|\nabla T \bar{\varphi}|^{2}\right)^{1 / 2} \leqslant C\left(\int_{T_{1}}|\nabla T \bar{\varphi}|^{2}\right)^{1 / 2}, \\
\int_{\partial B_{t}}\left|\frac{\partial \bar{\varphi}}{\partial \sigma}\right|^{2}+\frac{1}{2}\left(\frac{r_{0}}{\varepsilon}\right)^{2}\left(1-|\bar{\varphi}|^{2}\right)^{2} \\
\leqslant C \int_{T_{1}}\left|\frac{\partial \bar{\varphi}}{\partial \sigma}\right|^{2}+\frac{1}{2}\left(\frac{r_{0}}{\varepsilon}\right)^{2}\left(1-|\bar{\varphi}|^{2}\right)^{2}
\end{gathered}
$$


and

$$
\left(\int_{\partial B_{t}}|\nabla \bar{A}|^{p}\right)^{1 / p} \leqslant C\left(\int_{T_{1}}|\nabla \bar{A}|^{p}\right)^{1 / p} .
$$

We have also chosen $t$ such that

$$
\begin{aligned}
\int_{\partial B_{t r_{0}}} \frac{1}{\varepsilon^{2}} & \left(1-|\varphi|^{2}\right)^{2}=\int_{\partial B_{t}}\left(\frac{r_{0}}{\varepsilon}\right)^{2}\left(1-|\bar{\varphi}|^{2}\right)^{2} \\
& \leqslant C \int_{T_{1}}\left(\frac{r_{0}}{\varepsilon}\right)^{2}\left(1-|\bar{\varphi}|^{2}\right)^{2}=\frac{1}{r_{0}} \int_{B_{r_{0}}\left(x_{0}\right) \backslash B_{r_{0} / 2}\left(x_{0}\right)} \frac{1}{\varepsilon^{2}}\left(1-|\varphi|^{2}\right)^{2},
\end{aligned}
$$

and combined with (3. 7) the previous inequality gives

$$
\int_{\partial B_{t r_{0}}} \frac{1}{\varepsilon^{2}}\left(1-|\varphi|^{2}\right)^{2} \leqslant C \eta
$$

Since $\left\|\nabla_{\tilde{A}} \varphi\right\|_{\infty} \leqslant C / \varepsilon$ in $B_{r_{0}}\left(x_{0}\right)$ (from Lemma 2.4), we have, in particular, $\|\nabla|\varphi|\|_{\infty} \leqslant C / \varepsilon$ and, in view of (3. 32), for $\eta$ sufficiently small we have $|\varphi| \geqslant \frac{1}{2}$ on $\partial B_{t_{r_{0}}}\left(x_{0}\right)$ that is $|\bar{\varphi}| \geqslant \frac{1}{2}$ on $\partial B_{t}\left(x_{0}\right)$.

Since $H_{d R}^{1}\left(\partial B_{t}\left(x_{0}\right)\right)=0$ there exists $\bar{\psi} \in C^{\infty}\left(\partial B_{\rho}, \mathbb{R}\right)$ such that

$$
e^{i \bar{\psi}}=\frac{\bar{\varphi}}{|\bar{\varphi}|} \quad \text { on } \partial B_{\rho}\left(x_{0}\right) \quad \text { and } \quad \int_{\partial B_{t}} \bar{\psi} \in[0,2 \pi) \text {. }
$$

We have

$$
d_{\top} \bar{\psi}=\left(i \frac{\bar{\varphi}}{|\bar{\varphi}|}, d_{\top}\left(\frac{\bar{\varphi}}{|\bar{\varphi}|}\right)\right)=\frac{1}{|\bar{\varphi}|^{2}}\left(i \bar{\varphi}, d_{\top} \bar{\varphi}\right)
$$

On the other hand one verifies that for any $a \in C^{\infty}\left(\partial B_{t}, \mathbb{C}\right)$ we have

$$
(*) d_{\top}(*)\left(i a, d_{\top} a\right)=\left(i a, \Delta_{t} a\right)
$$

Applying (3. 34) to $\bar{\varphi}$ we have, using (3.16),

$$
\begin{gathered}
(*) d_{\top}(*)\left(i \bar{\varphi}, d_{\top} \bar{\varphi}\right)=\left(i \bar{\varphi}, \Delta_{\rho} \bar{\varphi}\right) \\
=-\frac{\partial}{\partial \sigma}\left(i \bar{\varphi} \frac{\partial \bar{\varphi}}{\partial \sigma}\right)-\frac{2}{\sigma}\left(i \bar{\varphi}, \frac{\partial \bar{\varphi}}{\partial \sigma}\right)+d^{*}\left(\hat{A}|\varphi|^{2}\right)
\end{gathered}
$$

We will replace, in $(3.35), d^{*}\left(\hat{A}|\bar{\varphi}|^{2}\right)$ by $d_{\top}^{(*)}\left(\hat{A}|\bar{\varphi}|^{2}\right)+\frac{\partial}{\partial \sigma}\left(\hat{A} \cdot \nu|\bar{\varphi}|^{2}\right)+\frac{2}{\sigma} \hat{A} \cdot \nu|\bar{\varphi}|^{2}$ and combining (3. 33) and (3. 35) we obtain

$$
\begin{aligned}
& \Delta_{t} \bar{\psi}=-\frac{\partial}{\partial \sigma}\left(i \bar{\varphi}, \frac{\partial \bar{\varphi}}{\partial \sigma}\right)-\frac{2}{\sigma}\left(i \bar{\varphi}, \frac{\partial \bar{\varphi}}{\partial \sigma}\right)+d_{\top}^{(*)}\left(\hat{A}|\varphi|^{2}\right)+\frac{\partial}{\partial \sigma}\left(\hat{A} \cdot \nu|\bar{\varphi}|^{2}\right) \\
&+\frac{2}{\sigma} \hat{A} \cdot \nu|\bar{\varphi}|^{2}+d_{\top}^{(*)}\left(\left(\frac{1}{|\bar{\varphi}|^{2}}-1\right)\left(i \bar{\varphi}, d_{\top} \bar{\varphi}\right)\right) \quad \text { on } \partial B_{t}\left(x_{0}\right) \\
& \text { ESaim: COCV June 1996, Vol.1, PP. } 77-167
\end{aligned}
$$


$\frac{\partial}{\partial \sigma}\left(i \bar{\varphi}, \frac{\partial \bar{\varphi}}{\partial \sigma}\right)$ looks a priori bad in the right hand side of $(3.36)$ in view of estimating $\bar{\psi}$ but using (3. 30) we have, using also Lemma A.1,

$$
\begin{aligned}
\frac{\partial}{\partial \sigma}\left(i \bar{\varphi}, \frac{\partial \bar{\varphi}}{\partial \sigma}\right) & =2 \frac{\partial}{\partial \sigma}<d V, \omega>+\frac{\partial H_{1}}{\partial \sigma}+\frac{\partial H_{2}}{\partial \sigma} \\
& =2<\frac{\partial}{\partial \sigma} d V, \omega>+\frac{\partial H_{1}}{\partial \sigma}+\frac{\partial H_{2}}{\partial \sigma} \\
& =2(*) d_{\top} \frac{\partial V}{\partial \sigma}-\frac{2}{\sigma}(*) d_{\top} V+\frac{\partial H_{1}}{\partial \sigma}+\frac{\partial H_{2}}{\partial \sigma} .
\end{aligned}
$$

Taking $q=\frac{4}{3}$ and $p=\frac{4}{3}$, classical elliptic estimates yield the following upper bound for $\int_{\partial B_{t}\left(x_{0}\right)}|\nabla T \bar{\psi}|^{4 / 3}$.

$$
\begin{aligned}
& \left(\int_{\partial B_{t}\left(x_{0}\right)}|\nabla+\psi|^{\frac{4}{3}}\right)^{3 / 4} \leqslant C\left[\left(\int_{\partial B_{t}\left(x_{0}\right)}|\nabla V|^{\frac{4}{3}}\right)^{3 / 4}\right. \\
& +\left(\int_{\partial B_{t}\left(x_{0}\right)}\left|\nabla H_{1}\right|^{\frac{4}{3}}\right)^{3 / 4}+\left(\int_{\partial B_{t}\left(x_{0}\right)}\left|\nabla H_{2}\right|^{2}\right)^{1 / 2} \\
& +\left(\int_{\partial B_{t}\left(x_{0}\right)}\left|\frac{\partial \bar{\varphi}}{\partial \sigma}\right|^{2}\right)^{1 / 2}+\left(\left.\left.\int_{\partial B_{t}\left(x_{0}\right)}|\hat{A}| \bar{\varphi}\right|^{2}\right|^{4 / 3}\right)^{3 / 4} \\
& +\left(\int_{\partial B_{t}\left(x_{0}\right)}\left|\frac{\partial}{\partial \sigma}\left(\hat{A} . \nu|\bar{\varphi}|^{2}\right)\right|^{4 / 3}\right)^{3 / 4} \\
& \left.+\left(\int_{\partial B_{t}\left(x_{0}\right)}\left(1-|\bar{\varphi}|^{2}\right)^{4 / 3}|\nabla \top \bar{\varphi}|^{4 / 3}\right)^{3 / 4}\right] .
\end{aligned}
$$

Combining (3. 20), (3. 27), (3. 29) and (3. 31), (3. 38) implies

$$
\begin{aligned}
& \left(\int_{\partial B_{t}\left(x_{0}\right)}\left|\nabla \nabla_{T}\right|^{\frac{4}{3}}\right)^{3 / 4} \leqslant \\
& \leqslant C\left[\left(\int_{T_{1}}|\nabla T \bar{\varphi}|^{2}\right)^{1 / 2}\left(\int_{T_{1}}\left|\frac{\partial \bar{\varphi}}{\partial \sigma}\right|^{2}\right)^{1 / 2}\right. \\
& +\left(\int_{T_{1}}\left|\frac{\partial \bar{\varphi}}{\partial \sigma}\right|^{2}\right)^{1 / 2}+\left(\int_{\partial T_{1}}\left|\frac{\partial \bar{\varphi}}{\partial \sigma}\right|^{2}\right)^{1 / 2} \\
& \left.+\left(\int_{T_{1}}|\nabla \hat{A}|^{2}\right)^{1 / 2}+\left(\int_{T_{1}}\left(1-|\bar{\varphi}|^{2}\right)^{4}\right)^{1 / 4}\left(\int_{T_{1}}\left|\nabla_{T} \bar{\varphi}\right|^{2}\right)^{\frac{1}{2}}+\eta\right],
\end{aligned}
$$

Esaim : Cocv June 1996, Vol.1, Pf. $77-167$ 
where we have also used the bound $\|\hat{A}\|_{\infty} \leqslant \frac{r_{0}}{d x_{0}}+r_{0} \eta^{1 / 2} \leqslant C \eta$ established above (see (3. 11). Let $\psi_{t}=\int_{\partial B_{t}\left(x_{0}\right)} \bar{\psi} \in[0,2 \pi)$ by Poincaré inequality and Sobolev embeding we have

$$
\left\|\bar{\psi}-\bar{\psi}_{t}\right\|_{H^{1 / 2}\left(\partial B_{t}\left(x_{0}\right)\right)} \leqslant C\left(\int_{\partial B_{t}\left(x_{0}\right)}\left|\nabla \nabla_{T} \bar{\psi}\right|^{4 / 3}\right)^{3 / 4} .
$$

Let $\tilde{\psi}$ be the harmonic extension of $\bar{\psi}$ in $B_{t}\left(x_{0}\right)$. We have from (3. 40) and classical results on harmonic functions,

$$
\left(\int_{B_{t}\left(x_{0}\right)}|\nabla \tilde{\psi}|^{2}\right)^{1 / 2} \leqslant C\left(\int_{\partial B_{t}\left(x_{0}\right)}|\nabla \bar{\psi} \bar{\psi}|^{4 / 3}\right)^{3 / 4}
$$

Let $\bar{\xi} B_{t}\left(x_{0}\right) \rightarrow \mathbb{R}_{+}$such that

$$
\left\{\begin{array}{l}
\bar{\xi}=|\bar{\varphi}| \quad \text { on } \partial B_{t}\left(x_{0}\right) \\
\bar{\xi} \text { is a minimizer of } \int_{\partial B_{t}\left(x_{0}\right)}|\nabla \bar{\xi}|^{2}+\left(\frac{r_{0}}{\varepsilon}\right)^{2}(1-\bar{\xi})^{2}
\end{array}\right.
$$

Clearly $\bar{\xi}$ is the solution of

$$
\begin{cases}-\left(\frac{\varepsilon}{r_{0}}\right)^{2} \Delta \bar{\xi}+\bar{\xi}=1 & \text { in } B_{t}\left(x_{0}\right) \\ \bar{\xi}=|\bar{\varphi}| & \text { on } \partial B_{t}\left(x_{0}\right) .\end{cases}
$$

By the maximum principle we have

$$
\max _{B_{t}\left(x_{0}\right)}(1-\bar{\xi}) \leqslant \max _{\partial B_{t}\left(x_{0}\right)}(1-|\bar{\varphi}|) \leqslant \frac{1}{2} .
$$

Multiply (3. 43) by $1-\bar{\xi}$ and integrate on $B_{t}\left(x_{0}\right)$, we have

$$
\int_{B_{t}\left(x_{0}\right)}|\nabla \bar{\xi}|^{2}+\left(\frac{r_{0}}{\varepsilon}\right)^{2}(1-\bar{\xi})^{2}=\int_{\partial B_{t}\left(x_{0}\right)}(1-\bar{\xi}) \frac{\partial}{\partial \nu}(1-\bar{\xi})
$$

and in view of (3. 44) this implies

$$
\begin{aligned}
\int_{B_{t}\left(x_{0}\right)}|\nabla \bar{\xi}|^{2} & +\left(\frac{r_{0}}{\varepsilon}\right)^{2}\left(1-\bar{\xi}^{2}\right)^{2} \\
& \leqslant C\left(\int_{\partial B_{t}\left(x_{0}\right)}\left(1-|\bar{\varphi}|^{2}\right)^{2}\right)^{1 / 2}\left(\int_{\partial B_{t}\left(x_{0}\right)}\left|\frac{\partial \bar{\xi}}{\partial \nu}\right|^{2}\right)^{1 / 2} .
\end{aligned}
$$

Multiply (3. 43) by $\sum_{i=1}^{3} x_{i} \frac{\partial(1-\bar{\xi})}{\partial x_{i}}$ and integrate on $B_{t}\left(x_{0}\right)$ we get the following Pohozaev identity

$$
\begin{aligned}
& \frac{1}{2} t \int_{\partial B_{t}\left(x_{0}\right)}|\nabla T \bar{\xi}|^{2}=\frac{1}{2} t \int_{\partial B_{t}\left(x_{0}\right)}\left|\frac{\partial \bar{\xi}}{\partial \nu}\right|^{2}+\frac{1}{2} \int_{B_{t}\left(x_{0}\right)}|\nabla \bar{\xi}|^{2}+ \\
&+\frac{3}{2} \int_{B_{t}\left(x_{0}\right)}\left(\frac{r_{0}}{\varepsilon}\right)^{2}(1-\bar{\xi})^{2}-\frac{t}{2} \int_{\partial B_{t}\left(x_{0}\right)}\left(\frac{r_{0}}{\varepsilon}\right)^{2}(1-\bar{\xi})^{2} . \\
& \text { Esaim : CoCV JUNe 1996, Vol.1, fr. } 77-167
\end{aligned}
$$


The Pohozaev identity and (3. 45) imply

$$
\int_{\partial B_{t}\left(x_{0}\right)}\left|\frac{\partial \bar{\xi}}{\partial \nu}\right|^{2} \leqslant\left. C \int_{\partial B_{t}\left(x_{0}\right)}\left|\nabla_{T}\right| \bar{\varphi}\right|^{2}+\left(\frac{r_{0}}{\varepsilon}\right)^{2}\left(1-|\bar{\varphi}|^{2}\right)^{2}
$$

(3. 46) combined with (3. 45), give

$$
\begin{aligned}
& \int_{\partial B_{t}\left(x_{0}\right)}|\nabla \bar{\xi}|^{2}+\left(\frac{r_{0}}{\varepsilon}\right)^{2}\left(1-\bar{\xi}^{2}\right)^{2} \\
& \leqslant C\left(\int_{\partial B_{t}}\left(1-|\bar{\varphi}|^{2}\right)^{2}\right)^{1 / 2}\left(\left.\int_{\partial B_{t}}|\nabla T| \varphi\right|^{2}+\left(\frac{r_{0}}{\varepsilon}\right)^{2}\left(1-|\bar{\varphi}|^{2}\right)^{2}\right)^{1 / 2} \\
& \leqslant C\left(\int_{T_{1}}\left(1-|\bar{\varphi}|^{2}\right)^{2}\right)^{1 / 2}\left(\int_{T_{1}}|\nabla \bar{\varphi}|^{2}+\left(\frac{r_{0}}{\varepsilon}\right)^{2}\left(1-|\bar{\varphi}|^{2}\right)^{2}\right)^{1 / 2}
\end{aligned}
$$

Let $\bar{W}=\bar{\xi} e^{i \tilde{\psi}}$ in $B_{t}\left(x_{0}\right)$, since $(\bar{\varphi}, \hat{A})$ minimizes $\bar{G}_{\varepsilon, r_{0}}$, we have $\bar{G}_{\varepsilon, r_{0}}(\bar{\varphi}, \hat{A}) \leqslant$ $\bar{G}_{\varepsilon, r_{0}}(\bar{W}, \hat{A})$ and this implies

$$
\begin{aligned}
& \int_{B_{t}\left(x_{0}\right)}\left|\nabla_{\hat{A}} \bar{\varphi}\right|^{2}+\left(\frac{r_{0}}{\varepsilon}\right)^{2}\left(1-|\bar{\varphi}|^{2}\right)^{2}+\frac{1}{r_{0}^{2}}|\bar{h}|^{2} \\
& \leqslant \int_{B_{t}\left(x_{0}\right)} 2|\nabla \tilde{\psi}|^{2}+|\nabla \bar{\xi}|^{2}+\left(\frac{r_{0}}{\varepsilon}\right)^{2}\left(1-|\bar{\xi}|^{2}\right)^{2}+2|\hat{A}|^{2}+\frac{1}{r_{0}^{2}}|\bar{h}|^{2}
\end{aligned}
$$

Combining (3. 39), (3. 41), (3. 47) and (3. 48) we have

$$
\begin{aligned}
\int_{B_{t}\left(x_{0}\right)} & \left|\nabla_{\hat{A}} \bar{\varphi}\right|^{2}+\left(\frac{r_{0}}{\varepsilon}\right)^{2}\left(1-|\varphi|^{2}\right)^{2}+\frac{1}{r_{0}^{2}}|\bar{h}|^{2} \\
& \leqslant C\left[\left(\int_{T_{1}}|\nabla T \bar{\varphi}|^{2}\right) \times\left(\int_{T_{1}}\left|\frac{\partial \bar{\varphi}}{\partial \sigma}\right|^{2}\right)+\int_{T_{1}}\left|\frac{\partial \bar{\varphi}}{\partial \sigma}\right|^{2}+\int_{\partial T_{1}}\left|\frac{\partial \bar{\varphi}}{\partial \sigma}\right|^{2}\right. \\
& +\left(\int_{T_{1}}\left(1-|\bar{\varphi}|^{2}\right)^{2}\right)^{1 / 2}\left(\int_{T_{1}}|\nabla \bar{\varphi}|^{2}+\left(\frac{r_{0}}{\varepsilon}\right)^{2}+\left(1-|\bar{\varphi}|^{2}\right)^{2}\right) \\
& \left.+\int_{T_{1}}|\nabla \hat{A}|^{2}+\eta^{2}+\int_{B_{t}\left(x_{0}\right)} \frac{1}{r_{0}^{2}}|\bar{h}|^{2}\right]
\end{aligned}
$$


Going back to the usual scale we have

$$
\begin{aligned}
\frac{1}{r_{0}} & E_{t r_{0}}\left(x_{0}\right) \leqslant C\left[\frac{1}{r_{0}} \int_{B_{r_{0}}}|\nabla \varphi|^{2} \times \frac{1}{r_{0}} \int_{B_{r_{0}}}\left|\frac{\partial \varphi}{\partial r}\right|^{2}+\frac{1}{r_{0}} \int_{B_{r_{0}}}\left|\frac{\partial \varphi}{\partial r}\right|^{2}\right. \\
& +\int_{\partial B_{r_{0}} \cup \partial B_{r_{0} / 2}}\left|\frac{\partial \varphi}{\partial r}\right|^{2}+r_{0} \int_{B_{r_{0}}}|\nabla \tilde{A}|^{2} \\
& +\left(\frac{1}{r_{0}^{3}} \int_{B_{r_{0}}}\left(1-|\varphi|^{2}\right)^{2}\right)^{1 / 2}\left(\frac{1}{r_{0}} \int_{B_{r_{0}}}|\nabla \varphi|^{2}+\frac{1}{\varepsilon^{2}}\left(1-|\varphi|^{2}\right)^{2}\right)^{2} \\
& \left.+\eta^{2}+\frac{1}{r_{0}} \int_{B_{t r_{0}}}|h|^{2}\right] .
\end{aligned}
$$

Since $|\tilde{A}| \leqslant \frac{1}{d_{x_{0}}}+C \eta^{1 / 2}$ (see 3 . 11), we have

$$
\frac{1}{r_{0}} \int_{B_{r_{0}}\left(x_{0}\right)}|\tilde{A}|^{2} \leqslant\left(\frac{r_{0}}{d_{x_{0}}}\right)^{2}+C r_{0}^{2} \eta^{1 / 2} \leqslant C \eta \text {. }
$$

Moreover $|\nabla \tilde{A}|^{2} \leqslant 2\left|\nabla A_{0}\right|^{2}+2|\nabla A|^{2}$ and we have $\left|\nabla A_{0}\right|^{2} \leqslant C / d_{x_{0}}^{4}$ and $\int_{\mathbb{R}^{3}}|\nabla A|^{2} \leqslant C \log \frac{1}{\varepsilon}$, thus

$$
r_{0} \int_{B_{r_{0}}}|\nabla \tilde{A}|^{2} \leqslant C\left(\frac{r_{0}^{4}}{d_{x_{0}}^{4}}+r_{0} \log 1 / \varepsilon\right) \leqslant C \eta
$$

Finally combining (3. 48), (3. 49), (3. 50) and (3. 51) we have

$$
\begin{aligned}
\frac{1}{r_{0}} E_{t_{0}}\left(x_{0}\right) & \leqslant \frac{E_{r_{0}}}{r_{0}} \times C \eta+C \eta+\left(\left(\frac{\varepsilon}{r_{0}}\right)^{2} C \eta\right)^{1 / 2} \frac{E_{r_{0}}}{r_{0}}+\eta^{2} \\
& \leqslant C \eta^{1 / 2}\left(\frac{E_{r_{0}}}{r_{0}}+1\right) .
\end{aligned}
$$

In the other hand we have by (3. 1)

$$
\frac{E_{r_{0}}}{r_{0}}-\frac{E_{t r_{0}}}{t r_{0}}=\int_{t r_{0}}^{r_{0}} \frac{J_{r}\left(x_{0}\right)}{r}+\int_{t r_{0}}^{r_{0}} R_{r}\left(x_{0}\right),
$$

we have also

$$
\int_{t r_{0}}^{r_{0}} \frac{J_{r}\left(x_{0}\right)}{r} \leqslant \frac{1}{t r_{0}} \int_{t r_{0}}^{r_{0}} J_{r}\left(x_{0}\right) \leqslant \frac{F_{r_{0}}\left(x_{0}\right)}{t r_{0}} \leqslant \frac{C}{t} \eta
$$

and furthermore, since we could have chosen $r_{0} \leqslant \frac{\eta}{\log 1 / \varepsilon}$ we have

$$
\begin{aligned}
\int_{t r_{0}}^{r_{0}} R_{r}\left(x_{0}\right) & \leqslant C r_{0} \log \frac{1}{\varepsilon}+C \frac{r_{0}^{2}}{d_{x_{0}}^{2}}\left(\frac{E_{r_{0}}\left(x_{0}\right)}{r_{0}}\right)^{1 / 2} \\
& \leqslant C \eta+C \eta\left(\frac{E_{r_{0}}\left(x_{0}\right)}{r_{0}}\right)_{\text {Esaim : Cocv June 1996, Vol.1, pr. } 77-167}^{1 / 2}
\end{aligned}
$$


Combining (3. 52), (3. 53), (3. 54) and (3. 55) we easily get that for $\eta$ sufficiently small, independently of $r_{0}$, we have

$$
\frac{E_{r_{0}}}{r_{0}}\left(x_{0}\right) \leqslant f(\eta) \quad \longrightarrow 0 \quad \text { as } \quad \eta \rightarrow 0
$$

This implies

$$
\begin{aligned}
\frac{E_{\varepsilon}}{\varepsilon}\left(x_{0}\right) & \leqslant f(\eta)+\int_{\varepsilon}^{r_{0}} R_{r}\left(x_{0}\right) \\
& \leqslant f(\eta)+C r_{0} \log \frac{1}{\varepsilon}+C \eta(f(\eta))^{1 / 2}
\end{aligned}
$$

and since we could have chosen $r_{0} \leqslant c \frac{\eta}{\log 1 / \varepsilon}$ we have

$$
\frac{1}{\varepsilon^{3}} \int_{B_{\varepsilon}\left(x_{0}\right)}\left(1-|\bar{\varphi}|^{2}\right)^{2} \leqslant \frac{E_{\varepsilon}}{\varepsilon} \leqslant g(\eta) \quad \longrightarrow 0 \quad \text { as } \eta \rightarrow 0
$$

and since $|\nabla| \varphi|| \leqslant\left|\nabla_{\tilde{A}} \varphi\right| \leqslant C / \varepsilon$ for $\eta$ sufficiently small independent of $\varepsilon$ (3. $56)$ ensures $|\varphi|\left(x_{0}\right) \geqslant \frac{1}{2}$. This proves the lemma.

\section{LOCATING THE SINGULARITIES}

As in [4], the bad set or the locus of the singulartities is by definition the place where $\left|\varphi_{\varepsilon}\right| \leqslant \frac{1}{2}$ and where we are going to have a loss of compactness (as we will see later). The Lemma 3.1 says that a point $x_{0}$ where $\left|\varphi_{\varepsilon}\right|\left(x_{0}\right)<\frac{1}{2}$ produces energy in the ball $B_{\rho}\left(x_{0}\right)$ greater than $\rho \times \eta \log \left(\frac{\rho}{\varepsilon}\right)$. In view of the global bound given by Lemma 2.1 we can easily obtain the three dimensional equivalent of Proposition 4.2 of [4], that is, we can put the bad set in $\frac{c(\alpha)}{\varepsilon^{\alpha}} 3$ balls of radius $\varepsilon^{\alpha}$ for $\alpha<1$, where $c(\alpha)$ only depends on $\alpha$ (this is equivalent to say, in dimension 2, that we can put the bad set in $c(\alpha) 2$-balls of radius $\varepsilon^{\alpha}$ ). Contrary to the dimension 2 (see [4], theorem 4.1 and [3] Lemma 4.1) we are not able to get the same result for $\alpha=1$, that is to give the location of the singularities at the scale $\varepsilon$ but as we will see it in part $V$ this is not necessary in view of getting global $W^{1, p}$ estimates.

We have the following technical Lemma, part 4 is devoted to the proof of this lemma.

Lemma 4.1. Given any $0<\alpha<1$ and $\alpha<\gamma<1$ there exist $N_{\alpha} \in \mathbb{N}$ and $\left(R_{i}\right)_{1 \leqslant i \leqslant N_{\alpha}}$ a finite sequence of disjoint rectangle sets whose boundary are unions of faces paralled to the planes $x O y, y O z$ and $x O z$ such that

$1-\exists, \mu>0, \forall 1 \leqslant i \leqslant N_{\alpha}, \forall x \in R_{i}$ (resp. $\forall y \in \mathbb{R}^{3} \backslash R_{i}$ ) there exists a cube of edge $\mu \varepsilon^{\alpha}$ included in $R_{i}$ (resp. in $\mathbb{R}^{3} \backslash R_{i}$ ) which contains $x$ (resp. $y$ ) and $R_{i}$ is contained in a cube of edge $2 \varepsilon^{\alpha}$ ( $\mu$ does not depend on $\varepsilon$ ). Those cubes have also faces parallel to the planes $x O y, y O z$ and $x O z$.

$2-N_{\alpha} \leqslant \frac{c(\alpha)}{\varepsilon^{\alpha}}$, where $c(\alpha)$ only depends on $\alpha$ and $\left\{\left(x_{i}, d_{i}\right)\right\}_{i=1, \ldots, n}$.

$3-\forall 1 \leqslant i \leqslant N_{\alpha} \quad \int_{\partial R_{i}}\left|\nabla_{\tilde{A}} \varphi\right|^{2}+\frac{1}{2 \varepsilon^{2}}\left(1-|\varphi|^{2}\right)^{2}+|h|^{2} \leqslant C \log \frac{1}{\varepsilon}$ where $C$ only depends on $\left\{\left(x_{l}, d_{l}\right)\right\}_{l=1, \ldots, n}$.

Esaim : CoCv June 1996, Vol.1, PP. 77-167 
$4-\forall 1 \leqslant i \leqslant N_{\alpha}$ there exists a subset $S_{i}$ of $\partial R_{i}$ (possibly empty) which can be contained in $n(\alpha, \gamma)$ balls of radius $\leqslant \varepsilon^{\gamma}$ such that

$$
|\varphi| \geqslant \frac{1}{2} \quad \text { on } \partial R_{i} \backslash S_{i}
$$

where $n(\alpha, \gamma)$ only depends on $\alpha, \gamma$ and $\left\{\left(x_{i}, d_{i}\right)\right\}_{i=1 \ldots n}$.

5 - for any $1 \leqslant i \leqslant N_{\alpha}$ such that $\forall k \in\{1, \ldots, n\} x_{k} \notin R_{i}$, for any regular closed curve in $\partial R_{i} \backslash S_{i}$ and for any trivialisation of $E$ over $R_{i}$, the degree of $\varphi /|\varphi|$ along this curve, which does not depend on the trivialisation chosen over $R_{i}$, is bounded by an integer $d(\alpha, \gamma)$ which only depends on $\alpha, \gamma$ and $\left\{\left(x_{i}, d_{i}\right)\right\}_{i=1 \ldots n}$. $6-$

$$
|\varphi| \geqslant \frac{1}{2} \quad \text { in } \quad \mathbb{R}^{3} \backslash \cup_{i=1}^{N_{k}} \bar{R}_{i} \cup_{k=1}^{n} C\left(\frac{\varepsilon^{\alpha}}{\eta}, x_{k}\right)
$$

where $\eta$ is given by Lemma 3.1 and $C\left(\frac{\varepsilon^{\alpha}}{\eta}, x_{k}\right)$ is the cube of centre $x_{k}$ of edge $\frac{\varepsilon^{\alpha}}{\eta}$ parallel to $O x, O y$ and $O z$.

\section{Proof of Lemma 4.1.}

Consider the lattice $\varepsilon^{\alpha} \mathbb{Z}^{3}$ in $\mathbb{R}^{3}$, and denote by $C(\rho, z)$ the cube of centre $z$, of edge $\rho$ and whose faces are parallel to $x O y, y O z$ and $x O z$.

We will only consider the points $z_{i}$ of the lattice such that $C\left(2 \varepsilon^{\alpha}, z_{i}\right)$ is not included in $\cup_{k=1}^{n}\left(C\left(\frac{\varepsilon^{\alpha}}{\eta}, x_{k}\right)\right)$. Let $J_{\alpha} \subset \varepsilon^{\alpha} \mathbb{Z}$ be this set. Let $\left(z_{i}\right)_{i \in I_{\alpha}}$ be those which verifies

$$
\frac{1}{2 \varepsilon^{\alpha}} \int_{C\left(2 \varepsilon^{\alpha}, z_{i}\right)}\left|\nabla_{\tilde{A}} \varphi\right|^{2}+\frac{1}{2 \varepsilon^{2}}\left(1-|\varphi|^{2}\right)^{2}+|h|^{2}>\eta \log \left(\frac{2 \varepsilon^{\alpha}}{\varepsilon}\right)
$$

where $\eta$ is the constant given by Lemma 3.1. We have

$$
\sum_{i \in I_{\alpha}} \int_{C\left(2 \varepsilon^{\alpha}, z_{i}\right)}\left|\nabla_{\tilde{A}} \varphi\right|^{2}+\frac{1}{2 \varepsilon^{2}}\left(1-|\varphi|^{2}\right)^{2}+|h|^{2}>\# I_{\alpha} 2 \varepsilon^{\alpha} \eta(1-\alpha) \log \frac{1}{\varepsilon} .
$$

Since any point of $\mathbb{R}^{3}$ is at most covered by $4^{3}$ of those cubes we have

$$
\int_{\mathbb{R}^{3} \backslash\left\{x_{1}, \ldots, x_{n}\right\}}\left|\nabla_{\tilde{A}} \varphi\right|^{2}+\frac{1}{2 \varepsilon^{2}}\left(1-|\varphi|^{2}\right)^{2}+|h|^{2}>\# I_{\alpha} C(1-\alpha) \varepsilon^{\alpha} \log \frac{1}{\varepsilon}(4.1)
$$

Combining (4. 1) and the global upper bound given by Lemma 2.1 we have

$$
\# I_{\alpha}<\frac{C}{(1-\alpha) \varepsilon^{\alpha}} .
$$

Moreover let $j \notin I_{\alpha}$, since

$$
\frac{1}{2 \varepsilon^{\alpha}} \int_{C\left(2 \varepsilon^{\alpha}, z_{j}\right)}\left|\nabla_{\tilde{A}} \varphi\right|^{2}+\frac{1}{2 \varepsilon^{2}}\left(1-|\varphi|^{2}\right)^{2}+|h|^{2} \leqslant \eta \log \left(\frac{\varepsilon^{\alpha}}{\varepsilon}\right),
$$

by Lemma $3.1,|\varphi| \geqslant 1 / 2$ in $C\left(\varepsilon^{\alpha}, z_{j}\right)$. In the other hand

$$
\mathbb{R}^{3} \backslash \underset{i \in I_{\alpha}}{\cup} C\left(\varepsilon^{\alpha}, z_{i}\right) \cup_{k=1}^{n} C\left(x_{k}, \frac{\varepsilon^{\alpha}}{\eta}\right) \underset{\substack{j \in J_{\alpha} \backslash I_{\alpha} \\ \text { Esaim : CoCv June 1996, Vol.1, pp. } 77-167}}{\subset} C\left(\varepsilon^{\alpha}, z_{j}\right)
$$


thus

$$
|\varphi| \geqslant \frac{1}{2} \quad \text { in } \mathbb{R}^{3} \backslash \bigcup_{i \in I_{\alpha}} C\left(\varepsilon^{\alpha}, z_{i}\right) \cup_{k=1}^{n} C\left(x_{k}, \frac{\varepsilon^{\alpha}}{\eta}\right) .
$$

We change now the covering of the bad set, $\left\{C\left(2 \varepsilon^{\alpha}, z_{i}\right)\right\}_{i \in I_{\alpha}}$ into a covering of cubes $\left\{C\left(\rho_{i}, z_{i}\right)\right\}_{i \in I_{\alpha}}$ with edges a little bit smaller than $2 \varepsilon^{\alpha}$ (ie $(2-\delta) \varepsilon^{\alpha} \leqslant \rho_{i} \leqslant 2 \varepsilon^{\alpha}, \delta$ will be fixed later) in view of ensuring conditions 3 , 4 and 5 of the lemma on the boundaries of the different connected components of $\mathbb{R}^{3} \backslash \cup_{i \in I_{\alpha}} \partial C\left(\rho_{i}, z_{i}\right)$ which will be rectangles verifying 1 .

Let $0<\delta<1$ chosen small enough at the end of the proof. Let $i \in I_{\alpha}$, from Lemma 2.5 there exists $C$ independent of $\varepsilon$ such that

$$
\frac{1}{2 \varepsilon^{\alpha}} \int_{C\left(2 \varepsilon^{\alpha}, z_{i}\right)}\left|\nabla_{\tilde{A}} \varphi\right|^{2}+\frac{1}{2 \varepsilon^{2}}\left(1-|\varphi|^{2}\right)^{2}+|h|^{2} \leqslant C \log \frac{1}{\varepsilon} .
$$

Let $\alpha<\beta<1$ and consider the lattice of size $\varepsilon^{\beta}$ (i.e. $\varepsilon^{\beta} \mathbb{Z}^{3}$ ) included in $C\left(2 \varepsilon^{\alpha}, z_{i}\right)$. Denote it by $\left(y_{j}\right)_{j \in J_{\alpha}^{\beta}\left(z_{i}\right)}$. Denote by $\left(y_{j}\right)_{j \in I_{\alpha}^{\beta}\left(z_{i}\right)}$ the points of this lattice such that

$$
\frac{1}{2 \varepsilon^{\beta}} \int_{C\left(2 \varepsilon^{\beta}, y_{j}\right)}\left|\nabla_{\tilde{A}} \varphi\right|^{2}+\frac{1}{2 \varepsilon^{2}}\left(1-|\varphi|^{2}\right)^{2}+|h|^{2}>\eta \log \left(\frac{2 \varepsilon^{\beta}}{\varepsilon}\right) .
$$

We have

$\sum_{j \in I_{\alpha}^{\beta}\left(z_{i}\right)} \int_{C\left(2 \varepsilon^{\beta}, y_{j}\right)}\left|\nabla_{\tilde{A}} \varphi\right|^{2}+\frac{1}{2 \varepsilon^{2}}\left(1-|\varphi|^{2}\right)^{2}+|h|^{2}>\# I_{\alpha}^{\beta}\left(z_{i}\right) 2 \varepsilon^{\beta}(1-\beta) \times \log \frac{1}{\varepsilon}$.

Since a point of $C\left(2 \varepsilon^{\alpha}, z_{i}\right)$ can be covered by, at most, $4^{3}$ cubes $C\left(2 \varepsilon^{\beta}, y_{j}\right)$ for $j \in J_{\alpha}^{\beta}\left(z_{i}\right)$ we have

$$
\begin{aligned}
\int_{C\left(z_{i}, 2 \varepsilon^{\alpha}\right)}\left|\nabla_{\tilde{A}} \varphi\right|^{2} & +\frac{1}{2 \varepsilon^{2}}\left(1-|\varphi|^{2}\right)^{2}+|h|^{2} \\
& >C \# I_{\alpha}^{\beta}\left(x_{i}\right) 2 \varepsilon^{\beta}(1-\beta) \log \frac{1}{\varepsilon} .
\end{aligned}
$$

Combining (4. 4) and (4. 5) we get

$$
\# I_{\alpha}^{\beta}\left(z_{i}\right)<\frac{C}{(1-\beta) \varepsilon^{\beta-\alpha}} .
$$

For $j \in J_{\alpha}^{\beta}\left(z_{i}\right) \backslash I_{\alpha}^{\beta}\left(z_{i}\right)$ we have, by Lemma 3.1,

$$
|\varphi| \geqslant \frac{1}{2} \quad \text { in } \quad C\left(\varepsilon^{\beta}, y_{j}\right)
$$

Moreover

$$
C\left(2 \varepsilon^{\alpha}, z_{i}\right) \backslash\left(\cup_{j \in I_{\alpha}^{\beta}\left(z_{i}\right)}^{\cup} C\left(\varepsilon^{\beta}, y_{j}\right)\right) \subset \underset{j \in J_{\alpha}^{\beta}\left(z_{i}\right) \backslash I_{\alpha}^{\beta}\left(z_{i}\right)}{\cup} C\left(\varepsilon^{\beta}, y_{j}\right)
$$

thus

$$
|\varphi| \geqslant \frac{1}{2} \quad \text { in } C\left(2 \varepsilon^{\alpha}, z_{i}\right) \backslash \cup_{j \in I_{\alpha}^{\beta}\left(z_{i}\right)} C\left(\varepsilon^{\beta}, y_{j}\right)
$$


Using the mean value formula we will choose $\rho \in\left((2-\delta) \varepsilon^{\alpha} ; 2 \varepsilon^{\alpha}\right)$ such that $\partial C\left(\rho, z_{i}\right)$ intersects a number of cubes $C\left(2 \varepsilon^{\beta}, y_{j}\right)$, for $j \in I_{\alpha}^{\beta}\left(z_{i}\right)$, bounded independently of $i, \varepsilon$ and such that

$$
\int_{\partial C\left(\rho, z_{i}\right)}\left|\nabla_{\tilde{A}} \varphi\right|^{2}+\frac{1}{2 \varepsilon^{2}}\left(1-|\varphi|^{2}\right)^{2}+|h|^{2} \leqslant K \log \frac{1}{\varepsilon},
$$

where $K$ only depends on $\delta$, and $\left\{\left(x_{i}, d_{i}\right)\right\}_{i=1, \ldots, n}$.

Let $\mathcal{N}_{\rho}\left(z_{i}\right)$ be the number of indices $j \in I_{\alpha}^{\beta}\left(z_{i}\right)$ such that

$$
C\left(2 \varepsilon^{\beta}, y_{j}\right) \cap \partial C\left(\rho, z_{i}\right) \neq \emptyset \text {. }
$$

If $\left|\rho_{1}-\rho_{2}\right|>2 \varepsilon^{\beta} \mathcal{N}_{\rho_{1}}\left(z_{i}\right)$ and $\mathcal{N}_{\rho_{2}}\left(z_{i}\right)$ count different indices thus, using (4. 6) we have

$$
\begin{aligned}
\int_{(2-\delta) \varepsilon^{\alpha}}^{2 \varepsilon^{\alpha}} \mathcal{N}_{\rho}\left(z_{i}\right) d \rho & \leqslant \sum_{p=0}^{\left[\frac{\delta \varepsilon^{\alpha}}{2 \varepsilon^{\beta}}\right]+1} \int_{2 \varepsilon^{\alpha}-2(p+1) \varepsilon^{\beta}}^{2 \varepsilon^{\alpha}-2 p \varepsilon^{\beta}} \mathcal{N}_{p}\left(z_{i}\right) d \rho \\
& \leqslant 2 \varepsilon^{\beta} \# I_{\alpha}^{\beta}\left(z_{i}\right) \leqslant \frac{C}{(1-\beta)} \varepsilon^{\alpha}
\end{aligned}
$$

In the other hand, (4. 4) gives

$$
\frac{\delta}{\varepsilon^{\alpha}} \int_{(2-\delta) \varepsilon^{\alpha}}^{2 \varepsilon^{\alpha}} d \rho \int_{\partial C\left(\rho, z_{i}\right)}\left|\nabla_{\tilde{A}} \varphi\right|^{2}+\frac{1}{2 \varepsilon^{2}}\left(1-|\varphi|^{2}\right)^{2}+|h|^{2} \leqslant K \log \frac{1}{\varepsilon}
$$

Applying the mean value formula, simultaneously for (4. 10) and (4. 11) we deduce the existence of $C$ independent of $i$ and $\varepsilon$ and the existence of $\rho_{i} \in\left((2-\delta) \varepsilon^{\alpha}, 2 \varepsilon^{\alpha}\right)$ such that

$$
\left\{\begin{array}{l}
\mathcal{N}_{\rho_{i}}\left(z_{i}\right) \leqslant C(\alpha, \beta, \delta) \\
\int_{\partial C\left(\rho_{i}, z_{i}\right)}\left|\nabla_{\tilde{A}} \varphi\right|^{2}+\frac{1}{2 \varepsilon^{2}}\left(1-|\varphi|^{2}\right)^{2}+|h|^{2} \leqslant C(\alpha, \beta, \delta) \log \frac{1}{\varepsilon}
\end{array}\right.
$$

We claim now that we can put the bad set $(|\varphi| \leqslant 1 / 2)$ on $\partial C\left(\rho_{i}, z_{i}\right)$ in a union of cubes of edges bounded by $\varepsilon^{\frac{\alpha+\beta}{2}}$ such that

1) the number of those cubes is still bounded by $\mathcal{N}_{\rho_{i}}\left(z_{i}\right) \leqslant C(\alpha, \beta, \delta)$

2 ) in any trivialisation of E over $C\left(2 \varepsilon^{\alpha}, x_{i}\right)$, the degree of $\varphi /|\varphi|$ along the intersection of $\partial C\left(\rho_{i}, z_{i}\right)$ and the union of those cubes is bounded independently of $\varepsilon$.

Let $j \in I_{\alpha}^{\beta}\left(z_{i}\right)$ such that $C\left(2 \varepsilon^{\beta}, y_{j}\right) \cap \partial C\left(\rho_{i}, z_{i}\right) \neq \emptyset$.

We claim that there exists $k \in\left[1 ; 2 \mathcal{N}_{\rho_{i}}\left(z_{i}\right)+2\right] \cap \mathbb{N}$ such that

$$
\begin{gathered}
|\varphi| \geqslant \frac{1}{2} \text { in } \\
C\left(\varepsilon^{\beta-\frac{k}{2} \frac{\beta-\alpha}{\left(2 \mathcal{N}_{\rho_{i}}+2\right)}}, y_{j}\right) \backslash C\left(\varepsilon^{\beta-\frac{(k-1)}{2} \frac{\beta-\alpha}{\left(2 \mathcal{N}_{\rho_{i}}+2\right)}}, y_{j}\right) \cap \partial C\left(\rho_{i}, z_{i}\right) \\
\text { Esaim : CoCv June 1996, Vol.1, pr. } 77-167
\end{gathered}
$$


Indeed any cube $C\left(2 \varepsilon^{\beta}, y_{j^{\prime}}\right)$ for $j^{\prime} \in I_{\alpha}^{\beta}\left(z_{i}\right)$ intersects at most two of the following annuli (because of its size)

$C\left(\varepsilon^{\beta-\frac{k}{2} \frac{\beta-\alpha}{\left(2 \mathcal{N} \rho_{i}+2\right)}}, y_{j}\right) \backslash C\left(\varepsilon^{\beta-\frac{(k-1)}{2} \frac{\beta-\alpha}{\left(2 \mathcal{N} \rho_{i}+2\right)}}, y_{j}\right)$ for $k \in\left\{1,2, \ldots, 2 \mathcal{N}_{\rho_{i}}+2\right\}$.

In fact, Since the number of the cubes $C\left(2 \varepsilon^{\beta}, y_{j^{\prime}}\right)\left(j^{\prime} \in I_{\alpha}^{\beta}\left(z_{i}\right)\right)$ which intersect $\partial C\left(\rho_{i}, x_{i}\right)$ is bounded by $\mathcal{N}_{\rho_{i}}$ there exists necessarily one of those annuli which intersects none of those cubes.

Let $k_{0} \in\left\{1,2, \ldots, 2 \mathcal{N}_{\rho_{i}}+2\right\}$ such that (4. 13) is verified.

For $r \in\left[\varepsilon^{\beta-\frac{\left(k_{0}-1\right)}{2} \frac{(\beta-\alpha)}{\left(2 N \rho_{i}+2\right)}}, \varepsilon^{\beta-\frac{k_{0}}{2} \frac{(\beta-\alpha)}{\left(2 \mathcal{N} \rho_{i}+2\right)}}\right]$, we denote by $\Gamma_{r}$ the following lipshitz closed curve in $\partial C\left(x_{i}, \rho_{i}\right)$

$$
\Gamma_{r}=\partial\left(C\left(r, y_{j}\right) \cap \partial C\left(\rho_{i}, z_{i}\right)\right)
$$

and $\Sigma_{r}$ the surface in $\partial C\left(\rho_{i}, z_{i}\right)$ that $\Gamma_{r}$ bounds

$$
\Sigma_{r}=C\left(r, y_{j}\right) \cap \partial C\left(\rho_{i}, z_{i}\right) .
$$

We have

$$
\begin{aligned}
\oint_{\Gamma_{r}} \frac{1}{|\varphi|^{2}}\left(i \varphi, \nabla_{\tilde{A}} \varphi\right) & =\oint_{\Gamma_{r}}\left(i \frac{\varphi}{|\varphi|}, \nabla \frac{\varphi}{|\varphi|}\right)-\oint_{\Gamma_{r}} \tilde{A} \\
& =2 \pi d-\int_{\Sigma_{r}} * \tilde{h} . \nu,
\end{aligned}
$$

where $d$ is the degree of $\varphi /|\varphi|$ on $\Gamma_{r}$ which is independent of

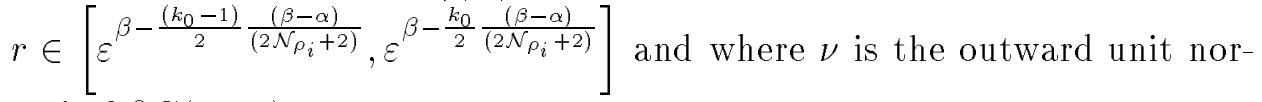
mal of $\partial C\left(\rho_{i}, z_{i}\right)$.

Since $|\varphi| \geqslant \frac{1}{2}$ on $\Gamma_{r}$ and $\left|\Gamma_{r}\right| \leqslant C r,(4.14)$ implies, using Cauchy-Schwartz inequality, that

$$
\frac{(2 \pi d)^{2}}{r} \leqslant C\left[\int_{\Gamma_{r}}\left|\nabla_{\tilde{A}} \varphi\right|^{2}+\frac{1}{r}\left(\int_{\sum_{r}}|\tilde{h}|\right)^{2}\right] .
$$

Moreover we have $|\tilde{h}| \leqslant\left|h_{0}\right|+|h|$ and

$$
\left|h_{0}\right| \leqslant \frac{C}{\operatorname{dist}^{2}\left\{y_{j} ;\left\{x_{i}, \ldots, x_{n}\right\}\right\}} \leqslant \frac{C}{\varepsilon^{2 \alpha}} .
$$

In the other hand, by (2. 38), we have

$$
\left(\int_{\Sigma_{r}}|h|\right)^{2} \leqslant C r^{2} \int_{\Sigma_{r}}|h|^{2} \leqslant C r^{3} \log \frac{1}{\varepsilon} .
$$

Combining (4. 15), (4. 16), (4. 17) and integrating with respect to $r$ we have

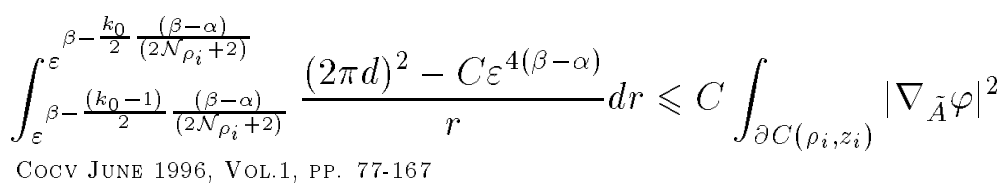

Esaim : Cocv June 1996, Vol.1, PP. 77-167 
In view of (4. 12) this implies

$$
\frac{\beta-\alpha}{2} \times \frac{1}{2 \mathcal{N}_{\rho_{i}}+2} 4 \pi^{2}\left(d^{2}-C \varepsilon^{4(\beta-\alpha)}\right) \leqslant C(\alpha, \beta, \delta) .
$$

Thus $d$ is bounded independently of $i$ and $\varepsilon$ and we have put the bad set on $\partial C\left(\rho_{i}, z_{i}\right)$ in at most $\mathcal{N}_{\rho_{i}}$ cubes of edge less than $\varepsilon^{\frac{\beta+\alpha}{2}}$ such that the degree of $\varphi /|\varphi|$ in any trivialisation of $E$ over $C\left(\rho_{i}, x_{i}\right)$ on the boundary of the intersection of those cubes with $\partial C\left(\rho_{i}, z_{i}\right)$ is uniformly bounded independently of $i$ and $\varepsilon$.

The connected components of $\mathbb{R}^{3} \backslash \cup_{i \in I_{\alpha}} \partial C\left(\rho_{i}, z_{i}\right)$ are sets which boundary is a union of faces parallel to $x O y, y O z$ and $z O x$ and for which conditions $2,3,4,5$ and 6 are verified for $\gamma=\frac{\beta+\alpha}{2}$. But there is no reason for condition 1 to be verified by this sequence of connected components. Some small changes have to be done in the proof to get 1 .

They appear here at the end of the proof in order not to overcomplicate the beginning.

Let $C(\rho, y)$ and $C\left(\rho^{\prime}, y^{\prime}\right)$ be two cubes with faces parallel to $x O y, y O z$ and $z O x$ and denote by

$$
d\left(C(\rho, y) ; C\left(\rho^{\prime}, y^{\prime}\right)\right)
$$

the minimum among all the distances between the faces of $\partial C(\rho, y)$ and the faces of $\partial C\left(\rho^{\prime}, y^{\prime}\right)$ which are parallel.

We are looking for a function $\lambda \varepsilon^{\alpha} \mathbb{Z}^{3} \rightarrow\left[2 ; 2+\frac{1}{2}\right]$ such that

$$
\begin{gathered}
\forall z, z^{\prime} \in \varepsilon^{\alpha} \mathbb{Z}^{3} y \neq y^{\prime} \\
d\left(C\left(\lambda(z) \varepsilon^{\alpha}, z\right) ; C\left(\lambda\left(z^{\prime}\right) \varepsilon^{\alpha}, z^{\prime}\right)\right) \geqslant \nu \varepsilon^{\alpha}
\end{gathered}
$$

for some $\nu>0$ independent of $\varepsilon$

Suppose such $\lambda \varepsilon^{\alpha} \mathbb{Z}^{3} \rightarrow\left[2,2+\frac{1}{2}\right]$ exists. Then in the previous proof, $\forall i \in I_{\alpha}$ choose $\rho_{i}$, by the mean value arguments developed above, between $\lambda\left(z_{i}\right) \varepsilon^{\alpha}$ and $\left(\lambda\left(z_{i}\right)-\delta\right) \varepsilon^{\alpha}$ (where $\delta$ is independent of $i$ and $\varepsilon$ ) instead of choosing $\rho_{i}$ between $2 \varepsilon^{\alpha}$ and $(2-\delta) \varepsilon^{\alpha}$. At the end of the proof, one comes exactly to the same conclusions, that is for $\mathbb{R}^{3} \backslash \cup_{i \in I_{\alpha}} \partial C\left(\rho_{i} ; z_{i}\right)$ the different connected components verify, 2, 3, 4, 5 and 6 , moreover if one have chosen $\delta<\nu / 4$ (where $\nu$ is given by (4. 20) ) since, in taking $\partial C\left(\rho_{i}, z_{i}\right)$ instead of taking $\partial C\left(\lambda\left(z_{i}\right) \varepsilon^{\alpha}, z_{i}\right)$, smaller than $\frac{\nu}{4} \varepsilon^{\alpha}$ we have

$$
\forall i, i^{\prime} \in I_{\alpha} i \neq i^{\prime} \quad d\left(C\left(\rho_{i}, z_{i}\right) ; C\left(\rho_{i^{\prime}}, z_{i^{\prime}}\right)\right) \geqslant\left(\nu-2 \frac{\nu}{4}\right) \varepsilon^{\alpha}=\frac{\nu}{2} \varepsilon^{\alpha}
$$

and

$$
\begin{gathered}
\forall i \in I_{\alpha}, \quad \forall i^{\prime} \notin I_{\alpha} \\
d\left(C\left(\rho_{i}, z_{i}\right) ; C\left(\lambda\left(z_{i^{\prime}}\right) \varepsilon^{\alpha}, z_{i^{\prime}}\right)\right) \geqslant\left(\nu-\frac{\nu}{4}\right) \varepsilon^{\alpha}=\frac{3 \nu}{4} \varepsilon^{\alpha} .
\end{gathered}
$$

(4. 21) and (4. 22) clearly ensure that the connected components of $\mathbb{R}^{3} \backslash \cup_{i \in I_{\alpha}} \partial C\left(\rho_{i}, z_{i}\right)$ verify condition 1 for $\mu=\nu / 2$ and the Lemma is proved. 
Thus, we just have to prove that $\lambda \varepsilon^{\alpha} \mathbb{Z}^{3} \rightarrow\left[2,2+\frac{1}{2}\right]$ verifying (4. 20) exists.

Take $\bar{\lambda} \varepsilon^{\alpha}\{1,2, \ldots, 5\}^{3} \rightarrow\left[2 ; 2+\frac{1}{4}\right]$ any injective function, and

$\lambda\left(\varepsilon^{\alpha}\left(n_{1}, n_{2}, n_{3}\right)\right)=\bar{\lambda}\left(\varepsilon^{\alpha}\left(\bar{n}_{1} ; \bar{n}_{2} ; \bar{n}_{3}\right)\right)$ where $\bar{n}_{l}$ is the representative of $n_{l}$ in $\mathbb{Z} / 5 \mathbb{Z}$ belonging to $\{1,2, \ldots, 5\}$.

We claim that (4. 20) holds for such $\lambda$

Letting $y, y^{\prime} \in \varepsilon^{\alpha} \mathbb{Z}$, two situations may occur

first case : $d\left(C\left(2 \varepsilon^{\alpha}, z\right) ; C\left(2 \varepsilon^{\alpha}, z^{\prime}\right)\right) \geqslant \varepsilon^{\alpha}$

by replacing $2 \varepsilon^{\alpha}$ by $\lambda \varepsilon^{\alpha}$, since $\lambda \in\left[2,2+\frac{1}{4}\right]$, we move each face of $C\left(2 \varepsilon^{\alpha}, z\right)$ and $C\left(2 \varepsilon^{\alpha}, z^{\prime}\right)$ parallely along a distance less than $\frac{\varepsilon^{\alpha}}{4}$ thus

$$
d\left(C\left(\lambda(z) \varepsilon^{\alpha}, z\right) ; C\left(\lambda\left(z^{\prime}\right) \varepsilon^{\alpha}, z^{\prime}\right)\right) \geqslant\left(1-\frac{2}{4}\right) \varepsilon^{\alpha}=\frac{\varepsilon^{\alpha}}{2}
$$

second case : $d\left(C\left(2 \varepsilon^{\alpha}, y\right), C\left(2 \varepsilon^{\alpha} ; y^{\prime}\right)\right)=0$

The parallel faces of $\overline{C\left(2 \varepsilon^{\alpha}, z\right)}$ and $\overline{C\left(2 \varepsilon^{\alpha}, z^{\prime}\right)}$ are either far from at least $\varepsilon^{\alpha}$ or touch themselves. For those which are far from at least $\varepsilon^{\alpha}$, replacing $2 \varepsilon^{\alpha}$ by $\lambda \varepsilon^{\alpha}$, as above, we keep the distance between themselves greater than $\varepsilon^{\alpha} / 2$. For the parallel faces of $\overline{C\left(2 \varepsilon^{\alpha}, z\right)}$ and $\overline{C\left(2 \varepsilon^{\alpha}, z^{\prime}\right)}$ which touch themselves we have $\max _{l=1,2,3}\left|z_{l}-z_{l}^{\prime}\right|=2 \varepsilon^{\alpha}$ and $\lambda$ has been chosen such that, for points which are so close, $\left|\lambda(z)-\lambda\left(z^{\prime}\right)\right| \geqslant \nu$ where

$$
\nu=\min \left\{\left|\bar{\lambda}(z)-\bar{\lambda}\left(z^{\prime}\right)\right|, z \neq z^{\prime} \quad z, z^{\prime} \in \varepsilon^{\alpha}\{1,2, \ldots, 5\}^{3}\right\} .
$$

Thus in replacing $2 \varepsilon^{\alpha}$ by $\lambda \varepsilon^{\alpha}$ the two parallel faces become distant by at least $\nu \varepsilon^{\alpha}$.

\section{5. $W^{1, p}$ ESTIMATES AND WEAK CONVERGENCE FOR THE MAGNETIC FIELD}

This part is devoted to the proof of theorem 5.1 stated below. First, we establish $W^{1, p}$ estimates for the renormalised magnetic field $h_{\varepsilon}=\tilde{h}_{\varepsilon}-h_{0}$. Precisely we have the following lemma

Lemma 5.1. Let $\left(\varphi_{\varepsilon}, \tilde{A}_{\varepsilon}\right)$ be a minimizer of $G_{\varepsilon}$ among $V$ and $h_{\varepsilon}=d \tilde{A}_{\varepsilon}-h_{0}$, we have

$$
\forall 1<p<\frac{3}{2} \quad \exists C_{p} \text { indep. of } \varepsilon \quad \text { s.t. } \quad \int_{\mathbb{R}^{3}}\left|\nabla h_{\varepsilon}\right|^{p} \leqslant C_{p}
$$

\section{Proof of Lemma 5.1.}

Let $0<\alpha<1$ and $\alpha<\gamma<1$ be fixed later. Let $\left(R_{i}\right)_{1 \leqslant i \leqslant N_{\alpha}}$ be the union of disjoint rectangle sets given by Lemma 4.1 and denote $T_{\varepsilon_{\alpha}}$ the following set

$$
T_{\varepsilon^{\alpha}}=\cup_{i=1}^{N_{\alpha}} \bar{R}_{i} \cup_{l=1}^{n} \bar{B}\left(x_{l}, \varepsilon^{\alpha} / \eta\right) .
$$

In $\mathbb{R}^{3} \backslash T_{\varepsilon^{\alpha}}$ we have $|\varphi| \geqslant \frac{1}{2}$. Taking the exterior derivative of $(2.15)$ we have

$$
-\Delta \tilde{h}+\tilde{h}=d\left[\left(1-\frac{1}{|\varphi|^{2}}\right)\left(i \varphi, d_{\tilde{A}} \varphi\right)\right] \quad \text { in } \mathbb{R}^{3} \backslash T_{\varepsilon^{\alpha}} .
$$


$T_{\varepsilon^{\alpha}}$ can be included in a big ball $B_{R_{\varepsilon}}$ of finite radius $R_{\varepsilon}<+\infty$ and we have

$$
\begin{aligned}
\forall 1<p<2 & \int_{\mathbb{R}^{3} \backslash B_{R_{\varepsilon}}}\left(1-\frac{1}{|\varphi|^{2}}\right)^{p}\left|\left(i \varphi, \nabla_{\tilde{A}} \varphi\right)\right|^{p} \leqslant \\
\leqslant & {\left[\int_{\mathbb{R}^{3} \backslash B_{R_{\varepsilon}}}\left(1-|\varphi|^{2}\right)^{\frac{2 p}{2-p}}\right]^{1-\frac{p}{2}} \times\left[\int_{\mathbb{R}^{3} \backslash B_{R_{\varepsilon}}}\left|\nabla_{\tilde{A}} \varphi\right|^{2}\right]^{\frac{p}{2}} } \\
\leqslant & C\left[\int_{\mathbb{R}^{3}}\left(1-|\varphi|^{2}\right)^{2}\right]^{1-\frac{p}{2}} \times\left[\int_{\mathbb{R}^{3}}\left|\nabla_{\tilde{A}} \varphi\right|^{2}\right]^{p / 2}<+\infty
\end{aligned}
$$

(5. 1) in $\mathbb{R}^{3} \backslash B_{R_{\varepsilon}}$ and (5. 2) yields by classical estimates on Bessel potential that

$$
\forall 1<p \leqslant 2 \quad \int_{\mathbb{R}^{3} \backslash B_{R_{\varepsilon}}}|\nabla \tilde{h}|^{p}<+\infty \quad .
$$

Moreover one easily verifies that $\nabla h_{0} \in L^{p}\left(\mathbb{R}^{3} \backslash B_{R_{\varepsilon}}\right)$ for any $p>1$. Thus

$$
\forall 1<p \leqslant 2 \quad \int_{\mathbb{R}^{3} \backslash B_{R_{\varepsilon}}}|\nabla h|^{p}<+\infty \quad,
$$

and since, from Lemma II.3, we have $h \in W^{1,2}\left(\mathbb{R}^{3}\right)$ we have

$$
\forall 1<p \leqslant 2 \quad \int_{\mathbb{R}^{3}}|\nabla h|^{p}<+\infty \quad .
$$

This implies, since $d^{*} \tilde{h}=d^{*} h$ in $\mathcal{D}^{\prime}\left(\mathbb{R}^{3}\right)$,

$$
\forall 1<p \leqslant 2 \quad \int_{\mathbb{R}^{3}}\left|d^{*} \tilde{h}\right|^{p}<+\infty \quad .
$$

We are going to establish a bound independent of $\varepsilon$ for $\int_{\mathbb{R}^{3}}\left|d^{*} \tilde{h}\right|^{p}$ for $1<$ $p<\frac{3}{2}$.

Let $0<\beta<1$ be fixed later and $K$ be the 1 -form on $\mathbb{R}^{3}$ solution of

$$
-\Delta K+K=\frac{d^{*} h}{\left|d^{*} h\right|^{\beta}} \quad \text { in } \mathcal{D}^{\prime}\left(\mathbb{R}^{3}\right)
$$

and $k=d K$ the two-form which verifies

$$
-\Delta k+k=d\left(\frac{d^{*} h}{\left|d^{*} h\right|^{\beta}}\right) \quad \text { in } \mathcal{D}^{\prime}\left(\mathbb{R}^{3}\right) \quad .
$$

We have

$$
\forall x \in \mathbb{R}^{3} \quad K(x)=c \int_{\mathbb{R}^{3}} \frac{d^{*} h}{\left|d^{*} h\right|^{\beta}}(y) \frac{e^{-|x-y|}}{|x-y|} d y .
$$

By classical estimates on Bessel potential we have for any $q$ such that $1<q(1-\beta) \leqslant 2$

$$
\int_{\mathbb{R}^{3}}|\nabla k|^{q} \leqslant C_{q} \int_{\mathbb{R}^{3}}\left|d^{*} h\right|^{q(1-\beta)}
$$

We will only work with $q>3$. Let $\delta=1-3 / q$. Sobolev injections give

$$
\forall x, y \in \mathbb{R}^{3} \quad|k(x)-k(y)| \leqslant C_{q}\left(\int_{\mathbb{R}^{3}}|\nabla k|^{q}\right)^{1 / q}|x-y|^{\delta} .
$$


Moreover

$$
\begin{aligned}
& \forall x \in \mathbb{R}^{3} \quad|k(x)|=|d K(x)| \\
& \leqslant C \int_{\mathbb{R}^{3}} \frac{e^{-|x-y|}}{|x-y|}\left(\frac{1}{|x-y|}+1\right)\left|d^{*} h\right|^{1-\beta}(y) d y
\end{aligned}
$$

and since $q>3, \quad q^{\prime}=\frac{q}{q-1}<\frac{3}{2}$ and $\forall x \in \mathbb{R}^{3}$ we have, $\forall x \in \mathbb{R}^{3}$,

$$
\begin{aligned}
|k(x)| & \leqslant\left(\int_{\mathbb{R}^{3}} \frac{e^{-q^{\prime}|x-y|}}{|x-y|^{q^{\prime}}}\left(\frac{1}{|x-y|^{q^{\prime}}}+1\right)\right)^{\frac{1}{q^{\prime}}} \times\left(\int_{\mathbb{R}^{3}} \frac{\left|d^{\star} h\right|^{q(1-\beta)}}{\left(1+\left|d^{\star} h\right|^{\beta}\right)^{q}}\right)^{1 / q} \\
& \leqslant C_{q}\left(\int_{\mathbb{R}^{3}}\left|d^{\star} h\right|^{q(1-\beta)}\right)^{1 / q}
\end{aligned}
$$

Multiply (5. 1) by $k$ and integrate on $\Omega_{\varepsilon^{\alpha}}=\mathbb{R}^{3} \backslash T_{\varepsilon^{\alpha}}$ we have

$$
\int_{\Omega_{\varepsilon} \alpha}-\left(k \cdot d d^{*} \tilde{h}+k \cdot d^{*} d \tilde{h}\right)+k \cdot \tilde{h}=\int_{\Omega_{\varepsilon} \alpha} k . d\left[\left(1-\frac{1}{|\varphi|^{2}}\right)\left(i \varphi, d_{\tilde{A}} \varphi\right)\right] .
$$

Integrating by parts on both sides of the equality and using the fact that $d \tilde{h} \equiv 0$ in $\Omega_{\varepsilon^{\alpha}}$ we have

$$
\begin{gathered}
-\int_{\partial T_{\varepsilon} \alpha} k \cdot d^{*} \tilde{h} \wedge \nu+\int_{\Omega_{\varepsilon} \alpha} d^{*} k \cdot d^{*} \tilde{h}+k \cdot d^{*} d \tilde{h}= \\
-\int_{\Omega_{\varepsilon} \alpha} d^{*} k \cdot\left(1-\frac{1}{|\varphi|^{2}}\right)\left(i \varphi, d_{\tilde{A}} \varphi\right)+\int_{\partial T_{\varepsilon} \alpha} k \cdot\left(1-\frac{1}{|\varphi|^{2}}\right)\left(i \varphi, d_{\tilde{A}} \varphi\right) \wedge \nu
\end{gathered}
$$

where $\nu$ is the 1 -form associated to the inward unit normal of $\partial T_{\varepsilon^{\alpha}}$. Multiplying (5. 7) by $\tilde{h}$ and integrating on all of $\mathbb{R}^{3}$ we have

$$
\int_{\mathbb{R}^{3}}-\left(\tilde{h} \cdot d d^{*} k+\tilde{h} \cdot d^{*} d k\right)+k \cdot \tilde{h}=\int_{\mathbb{R}^{3}} \tilde{h} \cdot d\left(\frac{d^{*} \tilde{h}}{\left|d^{*} \tilde{h}\right|^{\beta}}\right) .
$$

Integrating by parts on both sides of the equality and using the fact that $d k \equiv 0$ in $\mathbb{R}^{3}$ we have

$$
\int_{\mathbb{R}^{3}} d^{*} \tilde{h} \cdot d^{*} k+\tilde{h} \cdot k=-\int_{\mathbb{R}^{3}}\left|d^{*} \tilde{h}\right|^{2-\beta} .
$$

Combining (5. 12) and (5. 13) we have

$$
\begin{aligned}
& \int_{\mathbb{R}^{3}}\left|d^{*} \tilde{h}\right|^{2-\beta}=-\int_{\partial T_{\varepsilon} \alpha} k \cdot d^{*} \tilde{h} \wedge \nu-\int_{T_{\varepsilon} \alpha} d^{*} \tilde{h} \cdot d^{\star} k+\tilde{h} \cdot k \\
& -\int_{\partial T_{\varepsilon} \alpha} k \cdot\left(1-\frac{1}{|\varphi|^{2}}\right)\left(i \varphi, d_{\tilde{A}} \varphi\right) \wedge \nu+ \\
& +\int_{\Omega_{\varepsilon} \alpha} d^{*} k \cdot\left(1-\frac{1}{|\varphi|^{2}}\right)\left(i \varphi, d_{\tilde{A}} \varphi\right)
\end{aligned}
$$

We are going to establish some upper bounds for the four terms of the right hand side of (5. 14).

a) A bound for the fourth term of the r.h.s. of (5. 14).

Esaim: Cocv June 1996, Vol.1, Pp. 77-167 


$$
\begin{aligned}
\mid \int_{\Omega_{\varepsilon^{\alpha}}} & d^{*} k \cdot\left(1-\frac{1}{|\varphi|^{2}}\right)\left(i \varphi, d_{\tilde{A}} \varphi\right) \mid \\
& \leqslant C\left(\int_{\mathbb{R}^{3}}\left|d^{*} k\right|^{q}\right)^{1 / q} \times\left(\int_{\mathbb{R}^{3}}\left(1-|\varphi|^{2}\right)^{q^{\prime}}\left|\nabla_{\tilde{A}} \varphi\right|^{q^{\prime}}\right)^{1 / q^{\prime}} \\
& \leqslant C\left(\int_{\mathbb{R}^{3}}\left|d^{*} k\right|^{q}\right)^{1 / q} \times\left[\int_{\mathbb{R}^{3}}\left(1-|\varphi|^{2}\right)^{2}\right]^{\frac{1}{q^{\prime}}-\frac{1}{2}}\left[\int_{\mathbb{R}^{3}}\left|\nabla_{\tilde{A}} \varphi\right|^{2^{1}}\right]^{\frac{1}{2}} \\
& \leqslant C \varepsilon^{\frac{2}{q^{\prime}}-1}\left(\log \frac{1}{\varepsilon}\right)^{\frac{1}{q^{\prime}}}\left(\int_{\mathbb{R}^{3}}\left|d^{*} k\right|^{q}\right)^{1 / q}
\end{aligned}
$$

where $\frac{1}{q^{\prime}}=1-\frac{1}{q}$.

b) A bound for the third term of the r.h.s. of (5. 14).

$\left|\int_{\partial T_{\varepsilon} \alpha} k \cdot\left(1-\frac{1}{|\varphi|^{2}}\right)\left(i \varphi, d_{\tilde{A}} \varphi\right) \wedge \nu\right| \leqslant\|k\|_{L^{\infty}\left(\mathbb{R}^{3}\right)} \times \sum_{i=1}^{\mathcal{N}_{\alpha+n}} \int_{\partial R_{i}}\left|\nabla_{\tilde{A}} \varphi\right|\left(1-|\varphi|^{2}\right)$ $\left(\right.$ recall $R_{N_{\alpha}+l}=C\left(\varepsilon^{\alpha} / \eta, x_{l}\right)$ for $\left.1 \leqslant l \leqslant n\right)$.

We bound $\int_{\partial R_{i}}\left|\nabla_{\tilde{A}} \varphi\right|\left(1-|\varphi|^{2}\right)$ in the following way

$$
\begin{aligned}
\int_{\partial R_{i}}\left|\nabla_{\tilde{A}} \varphi\right|\left(1-|\varphi|^{2}\right) & \leqslant\left(\int_{\partial R_{i}}\left|\nabla_{\tilde{A}} \varphi\right|^{2}\right)^{1 / 2}\left(\int_{\partial R_{i}}\left(1-|\varphi|^{2}\right)^{2}\right)^{1 / 2} \\
& \leqslant C \varepsilon \log \frac{1}{\varepsilon}
\end{aligned}
$$

and since $N_{\alpha}+n \leqslant \frac{C(\alpha)}{\varepsilon^{\alpha}}$ we have, using also (5. 11),

$$
\left|\int_{\partial T_{\varepsilon} \alpha} k \cdot\left(1-\frac{1}{|\varphi|^{2}}\right)\left(i \varphi, d_{\tilde{A}} \varphi\right)\right| \leqslant C \varepsilon^{1-\alpha} \log \frac{1}{\varepsilon}\left(\int_{\mathbb{R}^{3}}\left|d^{*} h\right|^{q(1-\beta)}\right)^{1 / q}
$$

c) A bound for the second term of the r.h.s. of (5. 14).

We have $d^{*} \tilde{h}=d^{*} h$, thus

$$
\begin{aligned}
\left|\int_{T_{\varepsilon} \alpha} d^{*} h \cdot d^{*} k\right| & \leqslant\left(\int_{T_{\varepsilon} \alpha}\left|d^{*} h\right|^{2}\right)^{1 / 2}\left(\int_{T_{\varepsilon} \alpha}\left|d^{*} k\right|^{2}\right)^{1 / 2} \\
& \leqslant\left(\int_{T_{\varepsilon} \alpha}\left|\nabla_{\tilde{A}} \varphi\right|^{2}\right)^{1 / 2}\left|T_{\varepsilon^{\alpha}}\right|^{1-\frac{2}{q}}\left(\int_{\mathbb{R}^{3}}\left|d^{*} k\right|^{q}\right)^{1 / q}(5.17) \\
& \leqslant \varepsilon^{\alpha\left(2-\frac{4}{q}\right)}\left(\log \frac{1}{\varepsilon}\right)^{1 / 2}\left(\int_{\mathbb{R}^{3}}\left|d^{*} k\right|^{q}\right)^{1 / q} \cdot
\end{aligned}
$$


On the other hand $\int_{T_{\varepsilon} \alpha} \tilde{h} . k=\int_{T_{\varepsilon} \alpha} h_{0} . k+\int_{T_{\varepsilon} \alpha} h . k$ and we have

$$
\left|\int_{T_{\varepsilon} \alpha} h_{0} \cdot k\right| \leqslant\|k\|_{\infty}\left|\int_{T_{\varepsilon} \alpha}\right| h_{0}|| .
$$

Let $R<+\infty$ such that $\forall l \leqslant n \quad x_{l} \in B_{R}(0)$. Clearly $h_{0} \in L^{\infty}\left(\mathbb{R}^{3} \backslash B_{r}(0)\right)$, moreover $h_{0} \in L^{r}\left(B_{R}(0)\right)$ for $1 \leqslant r<\frac{3}{2}$. Thus

$$
\int_{T_{\varepsilon} \alpha}\left|h_{0}\right|=\int_{T_{\varepsilon} \alpha \cap B_{R}(0)}\left|h_{0}\right|+\int_{T_{\varepsilon} \alpha \cap\left[\mathbb{R}^{3} \backslash B_{R}(0)\right]}\left|h_{0}\right|
$$

and we have

$$
\int_{T_{\varepsilon^{\alpha}}}\left|h_{0}\right| \leqslant\left|T_{\varepsilon^{\alpha}}\right||| h_{0}\left\|_{L^{\infty}\left(\mathbb{R}^{3} \backslash B_{R}(0)\right)}+\left|T_{\varepsilon^{\alpha}}\right|^{1-\frac{1}{r}}|| h_{0}\right\|_{L^{r}\left(B_{R}(0)\right)} .
$$

Combining (5. 11), (5. 18) and the previous inequality we get for any $1<r<\frac{3}{2}$

$$
\left|\int_{T_{\varepsilon} \alpha} h_{0} \cdot k\right| \leqslant C \varepsilon^{2 \alpha\left(1-\frac{1}{r}\right)}\left(\int_{\mathbb{R}^{3}}\left|d^{*} h\right|^{q(1-\beta)}\right)^{1 / q} .
$$

Moreover, using also (5. 11),

$$
\begin{aligned}
\left|\int_{T_{\varepsilon} \alpha} h . k\right| & \leqslant|| k \|_{\infty}\left|T_{\varepsilon^{\alpha}}\right|^{1 / 2}\left(\int_{T_{\varepsilon} \alpha} h^{2}\right)^{1 / 2} \\
& \leqslant C \varepsilon^{\alpha}\left(\log \frac{1}{\varepsilon}\right)^{1 / 2}\left(\int_{\mathbb{R}^{3}}\left|d^{*} h\right|^{q(1-\beta)}\right)^{1 / q}
\end{aligned}
$$

d) A bound for the first term of the r.h.s. of (5. 14).

If $\nu$ denotes the inward normal for each $\left(R_{i}\right)_{i=1, \ldots, N_{\alpha}+n}$ we have by cancellation of $\nu$ and $-\nu$ on $\partial R_{i} \cap \partial R_{j}(i \neq j)$

$$
\begin{aligned}
\int_{\partial T_{\varepsilon} \alpha} k \cdot d^{*} \tilde{h} \wedge \nu & =-\int_{\partial\left(\cup_{i=1}^{N_{\alpha}+n} R_{i}\right)} k \cdot\left(i \varphi, d_{\tilde{A}} \varphi\right) \wedge \nu \\
& =\sum_{i=1}^{N_{\alpha}+n}-\int_{\partial R_{i}} k \cdot\left(i \varphi, d_{\tilde{A}} \varphi\right) \wedge \nu
\end{aligned}
$$

Denote by $\bar{k}_{i}$ for $i=1, \ldots, N_{\alpha}+n$ the mean value of $k$ on $R_{i}$ and by $\bar{k}$ the following approximation of $k$ in $T_{\varepsilon^{\alpha}}$

$$
\bar{k}=\sum_{i=1}^{N_{\alpha}+n} \bar{k}_{i} \chi\left(R_{i}\right)
$$

where $\chi\left(R_{i}\right)$ is the characteristic function of $R_{i}$ equal to 1 in $R_{i}$ and 0 elsewhere. Remark that from (5. 10) we deduce that

$$
\|k-\bar{k}\|_{L^{\infty}\left(T_{\varepsilon} \alpha\right)} \leqslant C_{q}\left(\int_{\mathbb{R}^{3}}\left|d^{*} k\right|^{q}\right)^{1 / q} \varepsilon^{\alpha \delta}
$$


where $\delta=1-\frac{3}{q}$. Moreover we have by $(5.21)$

$$
\begin{aligned}
\int_{\partial T_{\varepsilon} \alpha} & k \cdot d^{*} \tilde{h} \wedge \nu=\sum_{i=1}^{N_{\alpha}+n}-\int_{\partial R_{i}} k \cdot\left(i \varphi, d_{\tilde{A}} \varphi\right) \wedge \nu \\
& =\sum_{i=1}^{N_{\alpha}+n}-\int_{\partial R_{i}} \bar{k} \cdot\left(i \varphi, d_{\tilde{A}} \varphi\right) \wedge \nu+\int_{\partial R_{i}}(\bar{k}-k) \cdot\left(i \varphi, d_{\tilde{A}} \varphi\right) \wedge \nu
\end{aligned}
$$

The second term in the right hand side of $(5$. 23) can be bounded in the following way, using (5. 22) and condition 3 of Lemma 4.1,

$$
\begin{gathered}
\left|\sum_{i=1}^{N_{\alpha}+n} \int_{\partial R_{i}}(\bar{k}-k)\left(i \varphi, d_{\tilde{A}} \varphi\right) \wedge \nu\right| \leqslant|| k-\left.\bar{k}\right|_{L^{\infty}\left(T_{\varepsilon} \alpha\right)} \sum_{i=1}^{\mathcal{N}_{\alpha}+n} \int_{\partial R_{i}}\left|\nabla_{\tilde{A}} \varphi\right| \\
\leqslant C_{q}\left(\int_{\mathbb{R}^{3}}\left|d^{\star} k\right|^{q}\right)^{1 / q} \varepsilon^{\alpha \delta} \sum_{i=1}^{N_{\alpha}+n}\left|\partial R_{i}\right|^{1 / 2}\left(\log \frac{1}{\varepsilon}\right)^{1 / 2} .
\end{gathered}
$$

Since for $i=1, \ldots, \mathcal{N}_{\alpha}+n$ we have $\left|\partial R_{i}\right| \leqslant C \varepsilon^{2 \alpha}$ and $\mathcal{N}_{\alpha}+n \leqslant C(\alpha) / \varepsilon^{\alpha}$, the previous inequality implies

$$
\left|\sum_{i=1}^{N_{\alpha}+n} \int_{\partial R_{i}}(\bar{k}-k)\left(i \varphi, d_{\tilde{A}} \varphi\right) \wedge \nu\right| \leqslant C_{q}\left(\log \frac{1}{\varepsilon}\right)^{1 / 2} \varepsilon^{\alpha \delta}\left(\int_{\mathbb{R}^{3}}\left|d^{*} k\right|^{q}\right)^{1 / q}
$$

We establish now an upper bound for the first term of the right hand side of (5. 23). Consider now just the $R_{i}$ which do not contain any of the $\left(x_{l}\right)_{l=1, \ldots, n}$ since there are only $n$ which contain the $\left(x_{l}\right)$ the bound for them in (5. 23) is easy to establish.

We decompose the vector associated to the 1 -form $\star \bar{k}_{i}$ the canonical basis $\left(e_{1}, e_{2}, e_{3}\right)$ of $\mathbb{R}^{3}$, that is $\bar{k}_{i}^{1} e_{1}+\bar{k}_{i}^{2} e_{2}+\bar{k}_{i}^{3} e_{3}$. Thus we have

$$
\int_{\partial R_{i}} \bar{k} \cdot\left(i \varphi, d_{\tilde{A}} \varphi\right) \wedge \nu=\sum_{l=1}^{3} \bar{k}_{i}^{l} \int_{\partial R_{i}}\left(i \varphi, \nabla_{\tilde{A}} \varphi\right) \cdot \nu \wedge e_{l}
$$

Since $R_{i}$ is a set with faces perpendicular to $\epsilon_{1}, e_{2}$, and $\epsilon_{3}, \nu \wedge \epsilon_{1}, \nu \wedge \epsilon_{2}$ and $\nu \wedge e_{3}$ are unit tangent fields on $\partial R_{i}$ whose integral curves are the intersection of $\partial R_{i}$ with planes respectively perpendicular to $e_{1}, e_{2}$ and $e_{3}$. $\int_{\partial R_{i}}\left(i \varphi, \nabla_{\tilde{A}} \varphi\right) . \nu \wedge e_{l}$ for $l=1, \ldots, 3$ is the integral over all the planes perpendicular to $e_{l}$ intersecting $\partial R_{i}$ of the circulation of the current $\left(i \varphi, \nabla_{\tilde{A}} \varphi\right)$ along the integral curves of $\nu \wedge e_{l}$ that is the curves realized by the intersection of those planes perpendicular to $e_{l}$ with $\partial R_{i}$. Let $\hat{R}_{i}$ be the convex envelope of $R_{i}$. Write $\hat{R}_{i}=\Pi_{l=1}^{3}\left[r_{l}^{i}, s_{l}^{i}\right]$. Let $\Gamma_{l}(t)$ be the following curve

$$
\Gamma_{l}(t)=\partial R_{i} \cap\left\{x \in \mathbb{R}^{3} / x . e_{l}=t\right\} .
$$

$\Gamma_{l}(t)$ is oriented by $e_{l}$ (i.e. in the plane $\left\{x \in \mathbb{R}^{3} / x . e_{l}=t\right\}$ oriented by $e_{l} \Gamma_{l}(t)$ has the positive orientation). We have

$$
\int_{\partial R_{i}}\left(i \varphi, \nabla_{\tilde{A}} \varphi\right) \cdot \nu \wedge e_{l}=-\int_{t=r_{l}^{i}}^{s_{l}^{i}} \oint_{\Gamma_{l}(t)}\left(i \varphi, \nabla_{\tilde{A}} \varphi\right)
$$


For $i=1, \ldots, N_{\alpha}$ and $l=1,2,3$ denote $I_{l}^{i}=\left[r_{l}^{i}, s_{l}^{i}\right]$ and let $\tilde{I}_{l}^{i}$ be the subset of $t \in I_{l}^{i}$ such that $\Gamma_{l}(t)$ does not intersect the balls, given by Lemma 4.1, of radius bounded by $\varepsilon^{\gamma}$ which contain the bad set on $\partial R_{i}$. Since the number of those balls is bounded by $n(\alpha, \gamma)$ which does not depend neither on $i$ nor on $\varepsilon$, the measure of the surface $\Sigma_{l}^{i}=\cup_{t \notin \tilde{I}_{l}^{i}} \Gamma_{l}(t)$ is bounded in the following way

$$
\left|\Sigma_{l}^{i}\right| \leqslant C \varepsilon^{\gamma} \varepsilon^{\alpha}
$$

Using the definition of $\Sigma_{l}^{i}$ we have

$$
\int_{I_{l}^{i}} \oint_{\Gamma_{l}(t)}\left(i \varphi, \nabla_{\tilde{A}} \varphi\right)=\int_{\tilde{I}_{l}^{i}} \oint_{\Gamma_{l}(t)}\left(i \varphi, \nabla_{\tilde{A}} \varphi\right)+\int_{\sum_{l}^{i}}\left(i \varphi, \nabla_{\tilde{A}} \varphi\right) . \nu \wedge e_{l} .
$$

Using this decomposition of $\partial R_{i}$ we bound the first term of the right hand side of (5. 23) in the following way. First of all we have

$$
\begin{aligned}
& \mid \sum_{i=1}^{N_{\alpha}} \bar{k}_{l}^{i} \int_{\Sigma_{l}^{i}}\left(i \varphi, \nabla_{\tilde{A}} \varphi\right) \cdot \nu \wedge e_{l} \mid \\
& \leqslant C|| k \|_{L^{\infty}}\left[\sum_{i=1}^{N_{\alpha}+n}\left|\Sigma_{l}^{i}\right|^{1 / 2}\left(\int_{\partial R_{i}}\left|\nabla_{\tilde{A}} \varphi\right|^{2}\right)^{1 / 2}\right] \\
& \leqslant C\left[\int_{\mathbb{R}^{3}}\left|d^{*} \tilde{h}\right|^{q(1-\beta)}\right]^{1 / q} \frac{1}{\varepsilon^{\alpha}} \varepsilon^{\frac{\alpha}{2}+\frac{\gamma}{2}}\left(\log \frac{1}{\varepsilon}\right)^{1 / 2} \\
& \leqslant C \varepsilon^{\frac{\gamma-\alpha}{2}}\left(\log \frac{1}{\varepsilon}\right)^{1 / 2}\left[\int_{\mathbb{R}^{3}}\left|d^{*} \tilde{h}\right|^{q(1-\beta)}\right]^{1 / q} .
\end{aligned}
$$

On the good set on $\partial R_{i}$ we write

$$
\begin{aligned}
\int_{\tilde{I}_{l}^{i}} \oint_{\Gamma_{l}(t)}\left(i \varphi, \nabla_{\tilde{A}} \varphi\right) & =\int_{\tilde{I}_{l}^{i}} \oint_{\Gamma_{l}(t)}\left(i \frac{\varphi}{|\varphi|}, \nabla_{\tilde{A}} \frac{\varphi}{|\varphi|}\right)+ \\
& +\int_{\tilde{I}_{l}^{i}} \oint_{\Gamma_{l}(t)}\left(1-\frac{1}{|\varphi|^{2}}\right)\left(i \varphi, \nabla_{\tilde{A}} \varphi\right)
\end{aligned}
$$

and we have

$$
\begin{aligned}
& \qquad \begin{array}{ll}
\mid \sum_{i=1}^{N_{\alpha}} \bar{k}_{i}^{l} & \int_{\tilde{I}_{l}^{i}} \oint_{\Gamma_{l}(t)}\left(1-\frac{1}{|\varphi|^{2}}\right)\left(i \varphi, \nabla_{\tilde{A}} \varphi\right) \mid \\
& \leqslant C\|k\|_{L^{\infty}}\left[\sum_{i=1}^{\mathcal{N}_{\alpha}}\left(\int_{\partial R_{i}}\left(1-|\varphi|^{2}\right)^{2}\right)^{1 / 2}\left(\int_{\partial R_{i}}\left|\nabla_{\tilde{A}} \varphi\right|^{2}\right)^{1 / 2}\right] \\
& \leqslant C\left[\int_{\mathbb{R}^{3}}\left|d^{*} h\right|^{q(1-\beta)}\right]^{1 / q} \frac{1}{\varepsilon^{\alpha}} \varepsilon \log \frac{1}{\varepsilon} .
\end{array}
\end{aligned}
$$


Finally we have, for any trivialisation of $E$ over $R_{i}$,

$$
\begin{aligned}
\int_{\tilde{I}_{l}^{i}} \oint_{\Gamma_{l}(t)}\left(i \frac{\varphi}{|\varphi|}, \nabla_{\tilde{A}} \frac{\varphi}{|\varphi|}\right) & =\int_{\tilde{I}_{l}^{i}} \oint_{\Gamma_{l}(t)}\left(i \frac{\varphi}{|\varphi|}, \nabla \frac{\varphi}{|\varphi|}\right)-\int_{\tilde{I}_{l}^{i}} \oint_{\Gamma_{l}(t)} \tilde{A} \\
& =\int_{\tilde{I}_{l}^{i}} 2 \pi d_{l}(t)+\int_{\tilde{I}_{l}^{i}} \int_{S_{l}^{i}(t)} * \tilde{h} \cdot \nu
\end{aligned}
$$

where $d_{l}(t)$ is the degree of $\varphi /|\varphi|$ on $\Gamma_{l}(t)$ (which is bounded independently of $i$ and $\varepsilon$ by condition 5 of Lemma 4.1) and $S_{l}^{i}(t)$ is the surface in $\partial R_{i}$ which bounds $\Gamma_{l}(t)$ (taking into account the orientation of $\left.\Gamma_{l}(t)\right) . \partial R_{i}$ is of course oriented by the outward unit normal.

$$
\begin{aligned}
\left|\int_{S_{l}^{i}(t)} * \tilde{h} \cdot \nu\right| & \leqslant \int_{\partial R_{i}}\left|h_{0}\right|+\int_{\partial R_{i}}|h| \\
& \leqslant C\left[\int_{\partial R_{i}} \frac{1}{\varepsilon^{2 \alpha}}+\left|\partial R_{i}\right|^{1 / 2}\left(\log \frac{1}{\varepsilon}\right)^{1 / 2}\right] \leqslant C
\end{aligned}
$$

Thus combining (5. 32) and (5. 33) we have

$$
\begin{aligned}
\left|\sum_{i=1}^{N_{\alpha}} \bar{k}_{i}^{l} \int_{\tilde{I}_{l}^{i}} \oint_{\Gamma_{l}(t)}\left(i \frac{\varphi}{|\varphi|} ; \nabla_{\tilde{A}} \frac{\varphi}{|\varphi|}\right)\right| & \leqslant C\|k\|_{L^{\infty}}\left[\sum_{i=1}^{N_{\alpha}}\left|\tilde{I}_{l}^{i}\right|\right] \\
& \leqslant\left(\int_{\mathbb{R}^{3}}\left|d^{*} \tilde{h}\right|^{q(1-\beta)}\right)^{1 / q} \frac{1}{\varepsilon^{\alpha}} \times \varepsilon^{\alpha}
\end{aligned}
$$

Combining (5. 23), (5. 24), (5. 25), (5. 29), (5. 31) and (5. 34) we finally get an upper bound for the first term in the right hand side of $(5.14)$, namely

$$
\begin{aligned}
\left|\int_{\partial T_{\varepsilon} \alpha} k . d^{*} h \wedge \nu\right| & \leqslant\left(1+\varepsilon^{1-\alpha} \log \frac{1}{\varepsilon}+\varepsilon^{\gamma-\alpha}\left(\log \frac{1}{\varepsilon}\right)^{1 / 2}+\right. \\
& \left.+\varepsilon^{\alpha \delta}\left(\log \frac{1}{\varepsilon}\right)^{1 / 2}\right) \times\left(\int_{\mathbb{R}^{3}}\left|d^{*} \tilde{h}\right|^{q(1-\beta)}\right)^{\frac{1}{q}}
\end{aligned}
$$

Combining (5. 14), (5. 15), (5. 19), (5. 20) and (5. 35) we obtain for $\gamma>\alpha$, $q>3$ and $\varepsilon$ sufficiently small

$$
\int_{\mathbb{R}^{3}}\left|d^{*} \tilde{h}\right|^{2-\beta} \leqslant C(q, \gamma, \alpha)\left(\int_{\mathbb{R}^{3}}\left|d^{*} \tilde{h}\right|^{q(1-\beta)}\right)^{1 / q}
$$

where $C$ does not depend on $\varepsilon$.

Choose $\beta$ such that $2-\beta=q(1-\beta)$. Since $(5$. 36) holds for any $q>3$, this holds for any $1>\beta>\frac{1}{2}$. Thus for any $1<p<\frac{3}{2}$ we have

$$
\int_{\mathbb{R}^{3}}\left|d^{*} \tilde{h}\right|^{p} \leqslant C(p, \gamma, \alpha)\left(\int_{\mathbb{R}^{3}}\left|d^{*} \tilde{h}\right|^{p}\right)^{\frac{p-1}{p}},
$$


and we conclude that $\left\|d^{*} h\right\|_{L^{p}\left(\mathbb{R}^{3}\right)}$ is bounded independently of $\varepsilon$ for $1<$ $p<\frac{3}{2}$. By definition $A=-\frac{1}{4 \pi} \frac{1}{|x|} * d^{*} h$, thus by classical estimates on the Riesz transform, we have

$$
\int_{\mathbb{R}^{3}}|\nabla h|^{p} \leqslant \int_{\mathbb{R}^{3}}\left|\nabla^{2} A\right|^{p} \leqslant C_{p} \int_{\mathbb{R}^{3}}\left|d^{*} h\right|^{p} .
$$

This proves the Lemma.

From Lemma 5.1 we deduce the following useful corollary

Corollary 5.1. Let $\left(\varphi_{\varepsilon}, \tilde{A}_{\varepsilon}\right)$ be a minimizer of $G_{\varepsilon}$ among $V$. Then we have

$$
\begin{gathered}
\forall \Omega \subset \mathbb{R}^{3} \text { s. t. } \quad|\Omega|<+\infty \quad \forall 1<p<\frac{3}{2} \\
\int_{\Omega}\left|\nabla_{\tilde{A}} \varphi\right|^{p} \leqslant C(p,|\Omega|) .
\end{gathered}
$$

\section{Proof of corollary 5.1}

Let $0<\alpha<1$ and $T_{\varepsilon^{\alpha}}$ be the set introduced in the proof of Lemma 5.1. Since $|\varphi| \geqslant 1 / 2$ in $\mathbb{R}^{3} \backslash T_{\varepsilon^{\alpha}}$ we have

$$
\left|\nabla_{\tilde{A}} \varphi\right| \leqslant C\left(\left|\left(i \varphi, \nabla_{\tilde{A}} \varphi\right)\right|+|\nabla| \varphi||\right)
$$

From Lemma 5.1 and equation (2. 15) we deduce

$$
\forall 1<p<3 / 2 \quad \int_{\mathbb{R}^{3}}\left|\left(i \varphi, \nabla_{\tilde{A}} \varphi\right)\right|^{p} \leqslant C(p) \quad .
$$

On the other hand for any $p \in(1 ; 3 / 2)$

$$
\begin{aligned}
\int_{T_{\varepsilon} \alpha}\left|\nabla_{\tilde{A}} \varphi\right|^{p} & \leqslant\left|T_{\varepsilon^{\alpha}}\right|^{1-\frac{p}{2}}\left(\int_{T_{\varepsilon} \alpha}\left|\nabla_{\tilde{A}} \varphi\right|^{2}\right)^{\frac{p}{2}} \\
& \leqslant C \varepsilon^{\alpha(2-p)}\left(\log \frac{1}{\varepsilon}\right)^{\frac{p}{2}} \longrightarrow 0 \text { as } \varepsilon \rightarrow 0
\end{aligned}
$$

We bound $\int_{\Omega}|\nabla| \varphi||^{p}$ using a method developed in [3] (lemma X.13).

Recall equation (2. 14) verified by $|\varphi|^{2}$ in $\mathbb{R}^{3} \backslash\left\{x_{1}, \ldots, x_{n}\right\}$

$$
\frac{1}{2} \Delta|\varphi|^{2}=-\frac{|\varphi|^{2}}{\varepsilon^{2}}\left(1-|\varphi|^{2}\right)+\left|\nabla_{\tilde{A}} \varphi\right|^{2}
$$

Let $\overline{|\varphi|}=\max \left(|\varphi| ; 1-\frac{1}{\log \frac{1}{\varepsilon}}\right)$ and let

$$
K=\left\{x \in \mathbb{R}^{3} \backslash\left\{x_{1}, \ldots, x_{n}\right\} \text { s. } \mathrm{t}|\varphi| \geqslant 1-\frac{1}{\operatorname{Ln} \frac{1}{\varepsilon}}\right\} .
$$


Multiply (5. 42) by $1-\overline{|\varphi|}$ and integrate on $K \backslash \cup_{i=1}^{n} B\left(x_{i}, \varepsilon\right)$ we have

$$
\begin{aligned}
\left.\frac{1}{2} \int_{K \backslash \cup_{i=1}^{n} B\left(x_{i}, \varepsilon\right)}|\nabla| \varphi\right|^{2} \leqslant & \int_{K \backslash \cup_{i=1}^{n} B\left(x_{i}, \varepsilon\right)}\left|\nabla_{\tilde{A}} \varphi\right|^{2}(1-\overline{|\varphi|}) \\
& +\sum_{i=1}^{n} \int_{\partial B\left(x_{i}, \varepsilon\right)}|\nabla| \varphi||(1-\overline{|\varphi|}) \\
\leqslant & C \frac{\log 1 / \varepsilon}{\log 1 / \varepsilon}+n \frac{C}{\varepsilon} \frac{\varepsilon^{2}}{\log 1 / \varepsilon} \leqslant C .
\end{aligned}
$$

On the other hand

$$
\left|\mathbb{R}^{3} \backslash K\right| \times \frac{1}{\left(\log \frac{1}{\varepsilon}\right)^{2}} \leqslant \int_{\mathbb{R}^{3}}(1-|\varphi|)^{2} \leqslant C \varepsilon^{2} \log \frac{1}{\varepsilon}
$$

Let $p<2$, we have

$$
\begin{aligned}
\left.\int_{\mathbb{R}^{3} \backslash K}|\nabla| \varphi\right|^{p} & \leqslant\left|\mathbb{R}^{3} \backslash K\right|^{1-\frac{p}{2}} \times\left(\int_{\mathbb{R}^{3}}\left|\nabla_{\tilde{A}} \varphi\right|^{2}\right)^{\frac{p}{2}} \\
& \leqslant C \varepsilon^{2-p} \log \frac{1}{\varepsilon}
\end{aligned}
$$

The combination of (5. 39), (5. 40), (5. 41), (5. 43) and (5. 45) implies easily the desired result.

We are now in position to state and prove the following theorem which was the aim of the section 5 .

TheOREM 5.1. Let $\varepsilon_{n}$ be a sequence of positive numbers tending to 0 and $\left(\varphi_{\varepsilon_{n}}, \tilde{A}_{\varepsilon_{n}}\right)$ a sequence of minimizers of $G_{\varepsilon_{n}}$. One can extract a subsequence of $\varepsilon_{n}$ still denoted $\varepsilon_{n}$ such that there exists an integer multiplicity 1-dimensional rectifiable current $\mathbb{L}$ verifying

$$
\begin{gathered}
L=M\left(\mathbb{L}_{1}\right)<+\infty, \\
\partial \mathbb{L}_{d}=\sum_{i} d_{i}\left[\left|x_{i}\right|\right] \\
\mathcal{H}^{1}\left(\text { supp } \mathbb{L}_{1}\right)<+\infty
\end{gathered}
$$

and such that $h_{\varepsilon_{n}}=\tilde{h}_{\varepsilon_{n}}-h_{0} \rightarrow h_{\star}$ in $W_{l o c}^{1, p}\left(\mathbb{R}^{3}\right)$ for any $1<p<\frac{3}{2}$ where $h_{\star}$ verifies

$$
-\Delta h_{\star}+h_{\star}+h_{0}=-2 \pi * \mathbb{L}
$$

\section{Proof of theorem 5.1.}

Let $0<\alpha<1$ and $\alpha<\gamma<1$. Consider the domains $R_{i}, i \leqslant n+N_{\alpha}$, given by Lemma 4.1 using the notation

$$
\begin{aligned}
& R_{l+N_{\alpha}}=C\left(\varepsilon^{\alpha} / \eta, x_{l}\right) \text { for } l=1, \ldots, n . \\
& \text { Esaim : Cocv June 1996, Vol.1, PP. } 77-167
\end{aligned}
$$


By the same Lemma the set

$$
\left\{x \in \mathbb{R}^{3} \backslash\left\{x_{1}, \ldots, x_{n}\right\} \text { s.t. }|\varphi| \leqslant \frac{1}{2}\right\} \cap \underset{i=1 \ldots N_{\alpha}+n}{\cup} \partial R_{i}
$$

can be contained in $C(\alpha, \gamma) / \varepsilon^{\alpha}$ distinct balls of radius less than $\varepsilon^{\gamma}$. Moreover, since for each $I$ a number of those balls bounded by $C^{\prime}(\alpha, \gamma)$, independent of $\varepsilon$ and $i$, intersect $\partial R_{i}$. Provided we multiply the radius by some coefficient independent of $i$ and $\varepsilon$ we can consider that, if such a ball intersects $\partial R_{i}$, it has a centre on $\partial R_{i}$. Denote by $\left(a_{i}^{k}, r_{i}^{k}\right)_{k=1, \ldots, n_{i}}$ the centres and radii of the balls which intersect $\partial R_{i}$ ( $n_{i}$ is the number of those balls). Moreover denote by $d_{i}^{k}$ the degree of $\varphi /|\varphi|$ in any trivialisation of $E$ over $R_{i}$ (for $i \leqslant N_{\alpha}$ ) on the closed curve $\partial R_{i} \cap \partial B\left(a_{i}^{k}, r_{i}^{k}\right)$ oriented by the outward unit normal of $\partial R_{i}$. Lemma 4.1 says that $d_{i}^{k}$ is uniformly bounded independently of $\mathrm{i}$ and $\varepsilon$. For topological reasons we have

$$
\forall i \leqslant N_{\alpha} \quad \sum_{k=1}^{n_{i}} d_{i}^{k}=0
$$

For any $\mathrm{i}$ and $k \leqslant n_{i}, \partial R_{i} \cap \partial B\left(a_{i}^{k}, r_{I}^{k}\right)$ is a union of parts of circles. Each of these parts of circles belongs to the boundary of exactly two $R_{j}$ which have a face in common, but for the two $R_{j}$ the orientation of the face is opposite. Thus if one considers a trivialisation of $E$ over $\bar{B}\left(a_{i}^{k}, r_{i}^{k}\right)$ and if one adds all the degrees of $\varphi /|\varphi|$ on the $\cup_{j} \partial R_{j} \cap \partial B\left(a_{i}^{k}, r_{i}^{k}\right)$ for $j$ such that $a_{i}^{k} \in \partial B_{j}$, one will cover exactly two times the different parts of circles of $\cup_{j} \partial R_{j} \cap \partial B\left(a_{i}^{k}, r_{i}^{k}\right)$ but in the opposite sense and this implies

$$
\forall i \leqslant N_{\alpha} \quad \forall k \leqslant n_{i} \sum_{\left\{j \leqslant N_{\alpha}+n / \exists k_{j} \leqslant n_{j}\right.} \text { s.t. } a_{j}^{\left.k_{j}=a_{i}^{k}\right\}} d_{j}^{k_{j}}=0 .
$$

Let $i \leqslant N_{\alpha}$, since $(5.50)$ holds, one can establish a connection between the $\left(a_{i}^{k}, d_{i}^{k}\right)$ included in $R_{i}$, that is, there exists a finite union of on closed regular oriented curves included in $R_{i}$ with boundary included in $\cup_{i=1}^{n_{i}}\left\{a_{i}^{k}\right\}$ such that the 1 -dimensional integer multiplicity rectifiable current $\mathbb{L}_{i}^{\varepsilon}$ associated to this union of curves verify

$$
\partial \mathbb{L}_{i}^{\varepsilon}=\sum_{k=1}^{n_{i}} d_{i}^{k}\left[\left|a_{i}^{k}\right|\right]
$$

Moreover since $R_{i}$ verifies the condition 1 of Lemma 4.1, since from the conditions 4 and 5 of the same Lemma $d_{i}^{k}$ and $n_{i}$ are bounded independently of $i$ and $\varepsilon$ one can choose $\mathbb{L}_{i}^{\varepsilon}$ such that

$$
L_{i}^{\varepsilon}=M\left(\mathbb{L}_{i}^{\varepsilon}\right) \leqslant C(\alpha, \gamma) \varepsilon^{\alpha} .
$$

Let

$$
\mathbb{L}^{\varepsilon}=\sum_{i=1}^{N_{\alpha}} \mathbb{L}_{i}^{\varepsilon}
$$

since $\left|N_{\alpha}\right| \leqslant C(\alpha) / \varepsilon^{\alpha}$ we have

$$
L^{\varepsilon}=M\left(\mathbb{L}^{\varepsilon}\right) \leqslant C(\alpha, \gamma) \quad \text { indept. of } \varepsilon .
$$

Esaim : Cocv June 1996, Vol.1, PP. 77-167 
Moreover, from (5. 51) we deduce that

$$
\partial \mathbb{L}^{\varepsilon}=\sum_{l=1}^{n} \sum_{k=1}^{n_{N_{\alpha}+l}} d_{\mathcal{N}_{\alpha}+l}^{k}\left[\left|a_{N_{\alpha}+l}^{k}\right|\right]
$$

Contrary to the case $i \leqslant N_{\alpha}$ for $l=1, \ldots, n$ we cannot trivialize $E$ over $R_{N_{\alpha}+l}=C\left(\frac{\varepsilon^{\alpha}}{\eta}, x_{l}\right)$ thus $(5.50)$ does not hold in this case but we have that $h_{0} / 2 \pi$ is the Euler class of the restriction of the bundle $E$ over $\partial R_{N_{\alpha}+l}$ and theorem II.16 of [5] implies

$$
\forall l=1 \ldots n \quad \sum_{k=1}^{n_{N_{\alpha}+l}} d_{N_{\alpha}+l}^{k}=\frac{1}{2 \pi} \int_{\partial R_{N_{\alpha}+l}} h_{0}=d_{l}
$$

and this imply that

$$
\partial \mathbb{L}^{\varepsilon} \longrightarrow \sum_{l=1}^{n} d_{l}\left[\left|x_{l}\right|\right] \quad \text { as } \quad \varepsilon \rightarrow 0
$$

Moreover since $n_{N_{\alpha}+l}$ and $d_{N_{\alpha}+l}$ are uniformly bounded independently of $\varepsilon$ we have

$$
M\left(\partial \mathbb{L}^{\varepsilon}\right) \leqslant C \quad \text { indep. of } \varepsilon .
$$

Let $\varepsilon_{n}$ be a sequence of positive number tending to 0 , from $(5$. 55) and (5. 59), using Federer-Fleming's compactness theorem (see for instance [9] page 97 ), we deduce the existence of a 1-dimensional, integer multiplicity rectifiable current $\mathbb{L}$ and a subsequence still denoted $\varepsilon_{n}$ such that

$$
\mathbb{L}^{\varepsilon_{n}} \rightarrow \mathbb{L} \quad \text { in } \quad \mathcal{D}_{1}\left(\mathbb{R}^{3}\right)
$$

Moreover from (5. 58) we deduce that

$$
\partial \mathbb{L}_{s}=\sum_{l=1}^{n} d_{l}\left[\left|x_{l}\right|\right]
$$

From Lemma 5.1 we know that for any $1<p<\frac{3}{2}, \int_{\mathbb{R}^{3}}\left|\nabla h_{\varepsilon_{n}}\right|^{p}<C$ independently of $\varepsilon$ and since $h_{\varepsilon_{n}} \in L^{\frac{3 p}{p-3}}\left(\mathbb{R}^{3}\right)$ we have

$$
\forall 1<p<\frac{3}{2} \quad \int_{\mathbb{R}^{3}}\left|h_{\varepsilon_{n}}\right|^{\frac{3 p}{p-3}}+\left|\nabla h_{\varepsilon_{n}}\right|^{p} \leqslant C \quad \text { indep. of } \varepsilon .
$$

One can extract a subsequence still denoted $\varepsilon_{n}$ such that (5. 60) holds and

$$
\text { for } \quad 1<p<\frac{3}{2} \quad h_{\varepsilon_{n}} \rightarrow h_{\star} \quad \text { weakly in } W_{l o c}^{1, p}\left(\mathbb{R}^{3}\right) \text {. }
$$

Let $\psi$ be a $C^{\infty} 1$-form on $\mathbb{R}^{3}$ with compact support. Our aim, now, is to prove

$$
\int_{\mathbb{R}^{3}} * d \psi \cdot d^{*} h_{\varepsilon_{n}}+* \psi \cdot \tilde{h}_{\varepsilon_{n}}+<2 \pi \mathbb{L}^{\varepsilon_{n}}, \psi>\longrightarrow 0
$$

as $n$ tends to infinity. In view of (5. 60) and (5. 62) that will imply (5. 49). We use the notation $h_{n}, A_{n}, \varphi_{n}, L^{n}$, for $h_{\varepsilon_{n}}, A_{\varepsilon_{n}}, \varphi_{\varepsilon_{n}}$ and $L^{\varepsilon_{n}}$. Recall that on $\mathbb{R}^{3} \backslash T_{\varepsilon_{n}^{\alpha}}$ we have

$$
-d d^{\star} h_{n}+\tilde{h}_{n}=d\left[\left(1-\frac{1}{\left|\varphi_{n}\right|^{2}}\right)\left(i \varphi_{n}, d_{\tilde{A}_{n}} \varphi\right)\right] \text {. }
$$


Multiplying (5. 64) by $* \psi$ and integrating on $\Omega_{\varepsilon_{n}^{\alpha}}=\mathbb{R}^{3} \backslash T_{\varepsilon_{n}^{\alpha}}$ we get

$$
\begin{gathered}
\int_{\Omega_{\varepsilon_{n}^{\alpha}}} * d \psi \cdot d^{*} h_{n}+\int_{\partial_{\varepsilon_{n}^{\alpha}}} * \psi \cdot d^{*} h_{n} \wedge \nu+\int_{\Omega_{\varepsilon_{n}^{\alpha}}} * d \psi \cdot \tilde{h} \\
=-\int_{\Omega_{\varepsilon_{n}^{\alpha}}}\left(1-\frac{1}{\left|\varphi_{n}\right|^{2}}\right) d \psi \cdot *\left(i \varphi_{n}, d_{\tilde{A}_{n}} \varphi_{n}\right) \\
\quad+\int_{\partial \Omega_{\varepsilon_{n}^{\alpha}}}\left(1-\frac{1}{\left|\varphi_{n}\right|^{2}}\right) * \psi \cdot\left(i \varphi_{n}, d_{\tilde{A}_{n}} \varphi_{n}\right) \wedge \nu
\end{gathered}
$$

where $\nu$ denotes the 1-form associated to the unit inward normal of $\partial T_{\varepsilon_{n}^{\alpha}}$. Since $d^{*} h_{n}$ and $\tilde{h}_{n}$ converges in $L^{p}(\operatorname{supp} \psi)$ and since $\left|T_{\varepsilon_{n}^{\alpha}}\right|=\left|\mathbb{R}^{3} \backslash \Omega_{\varepsilon_{n}^{\alpha}}\right| \rightarrow 0$ we have

$$
\int_{\Omega_{\varepsilon_{n}^{\alpha}}} * d \psi \cdot d^{*} h_{n}+\int_{\Omega_{\varepsilon_{n}^{\alpha}}} * \psi \cdot \tilde{h}_{n} \underset{n \rightarrow+\infty}{\longrightarrow} \int_{\mathbb{R}^{3}} * d \psi \cdot d^{*} h_{\star}+* \psi \cdot \tilde{h}_{\star}
$$

Moreover $\left(1-\left|\varphi_{n}\right|^{2}\right) \rightarrow 0$ in $L^{q}\left(\mathbb{R}^{3}\right) \quad(2 \leqslant q<\infty)$ and $\left(i \varphi_{n}, d_{\tilde{A}_{n}} \varphi_{n}\right)=$ $-d^{*} h_{n}$ is bounded in $L^{p}\left(\mathbb{R}^{3}\right) \quad\left(1<p<\frac{3}{2}\right)$, thus

$$
\left.\int_{\Omega_{\varepsilon_{n}^{\alpha}}}|1-| \varphi_{n}\right|^{2}|| d \psi||\left(i \varphi_{n}, d_{\tilde{A}_{n}} \varphi_{n}\right) \mid \underset{n \rightarrow+\infty}{\longrightarrow} 0
$$

\section{Moreover}

$$
\begin{aligned}
\mid \int_{\partial T_{\varepsilon_{n}^{\alpha}}} & \left(1-\frac{1}{\left|\varphi_{n}\right|^{2}}\right) * \psi \cdot\left(i \varphi_{n}, d_{\tilde{A}_{n}} \varphi_{n}\right) \wedge \nu \mid \\
& \leqslant C\|\psi\|_{\infty} \sum_{i=1}^{N_{\alpha}+n} \int_{\partial R_{i}}\left(1-\left|\varphi_{n}\right|^{2}\right)\left|\nabla_{\tilde{A}_{n}} \varphi_{n}\right| \\
& \leqslant C\|\psi\|_{\infty} \sum_{i=1}^{N_{\alpha}+n}\left(\left.\left.\int_{\partial R_{i}}|1-| \varphi_{n}\right|^{2}\right|^{2}\right)^{1 / 2}\left(\int_{\partial R_{i}}\left|\nabla_{\tilde{A}_{n}} \varphi_{n}\right|^{2}\right)^{1 / 2} \\
& \leqslant C\|\psi\|_{\infty} \frac{1}{\varepsilon_{n}^{\alpha}} \varepsilon_{n} \log \frac{1}{\varepsilon_{n}} \underset{n \rightarrow+\infty}{\longrightarrow} 0
\end{aligned}
$$

Let $\nu$ denotes the 1 -form associated to the unit inward normal to $\partial R_{i}$, by cancelation on common faces we have

$$
-\int_{\partial T_{\varepsilon_{n}^{\alpha}}} * \psi \cdot d^{*} h_{n} \wedge \nu=+\sum_{i=1}^{N_{\alpha}+n} \int_{\partial R_{i}} * \psi \cdot\left(i \varphi_{n}, d_{\tilde{A}_{n}} \varphi_{n}\right) \wedge \nu
$$

Let $\bar{\psi}_{i}$ be the mean value of $\psi$ on $R_{i}$, we have

$$
\left\|\psi-\bar{\psi}_{i}\right\|_{L^{\infty}\left(R_{i}\right)} \leqslant C\|\nabla \psi\|_{\infty} \varepsilon^{\alpha} .
$$


and

$$
\begin{aligned}
\sum_{i=1}^{N_{\alpha}+n} \int_{\partial R_{i}}\left|\psi-\bar{\psi}_{i}\right| & \left|\left(i \varphi_{n}, d_{\tilde{A}_{n}} \varphi_{n}\right)\right| \\
& \leqslant C\|\nabla \psi\|_{\infty} \sum_{i=1}^{N_{\alpha}+n} \varepsilon_{n}^{\alpha}\left|\partial R_{i}\right|^{1 / 2}\left(\int_{\partial R_{i}}\left|\nabla_{\tilde{A}_{n}} \varphi\right|^{2}\right)^{1 / 2}(5.71) \\
& \leqslant C\|\nabla \psi\|_{\infty} \varepsilon_{n}^{\alpha}\left(\log \frac{1}{\varepsilon_{n}}\right)^{1 / 2} \rightarrow 0 \text { as } n \rightarrow+\infty
\end{aligned}
$$

We are led to study closely the quantities

$$
I_{i}=\int_{\partial R_{i}} * \bar{\psi}_{i} \cdot\left(i \varphi_{n}, d_{\tilde{A}_{n}} \varphi_{n}\right) \wedge \nu \quad \text { for } \quad i \leqslant N_{\alpha}
$$

Use vector instead of 1 -forms ( ie $\bar{\psi}_{i}$ denotes also the associated vector to the 1 -form $\bar{\psi}_{i}$ ).

$$
I_{i}=\int_{\partial R_{i}} * \bar{\psi}_{i} \cdot\left(i \varphi_{n}, d_{\tilde{A}_{n}} \varphi_{n}\right) \wedge \nu=\int_{\partial R_{i}}\left(i \varphi, \nabla_{\tilde{A}_{n}} \varphi\right) \cdot \nu \wedge \bar{\psi}_{i}
$$

Decompose $\bar{\psi}_{i}$ in the canonical basis of $\mathbb{R}^{3}, \bar{\psi}_{i}=\sum_{l=1}^{3} \bar{\psi}_{i}^{l} e_{l}$. We have

$$
I_{i}=\sum_{l=1}^{3} \bar{\psi}_{i}^{l} \int_{\partial R_{i}}\left(i \varphi_{n}, \nabla_{\tilde{A}_{n}} \varphi_{n}\right) \cdot \nu \wedge \epsilon_{l}
$$

Let $\hat{R}_{i}=\prod_{l=1}^{3}\left[r_{i}^{l} ; s_{i}^{l}\right]$ be the convex envelope of $R_{i}$ and let $\Gamma_{l}(t)=\partial R_{i} \cap\{x \in$ $\left.\mathbb{R}^{3} / x . e_{l}=t\right\}$ as in the proof of Lemma 5.1 (see (5.25)) we have

$$
\int_{\partial R_{i}}\left(i \varphi_{n}, \nabla_{\tilde{A}} \varphi_{n}\right) \cdot \nu \wedge e_{l}=-\int_{t=r_{i}^{l}}^{s_{i}^{l}} \oint_{\Gamma_{l}(t)}\left(i \varphi_{n}, \nabla_{\tilde{A}_{n}} \varphi_{n}\right)
$$

where $\Gamma_{l}(t)$ is oriented by $e_{l}$ (i.e. in the plane $\left\{x \in \mathbb{R}^{3} / x . e_{l}=t\right\}$ oriented by $e_{l}, \Gamma_{l}(t)$ has the positive orientation). Denote by $\tilde{I}_{i}^{l}$ the following interval

$$
\tilde{I}_{i}^{l}=\left[r_{i}^{l} ; s_{i}^{l}\right] \backslash \cup_{k=1}^{n_{i}}\left(a_{i}^{k, l}-\varepsilon_{n}^{\gamma} ; a_{i}^{k, l}+\varepsilon_{n}^{\gamma}\right)
$$

where $a_{i}^{k, l}=a_{i}^{k} \cdot e_{l}$. On $\Gamma_{l}(t)$ for $t \in \tilde{I}_{i}^{l}$ we have $|\varphi| \geqslant \frac{1}{2}$. Moreover the surface

$$
\Sigma_{i}^{l}=\underset{t \in\left[r_{i}^{l}: s_{i}^{l}\right] \backslash \tilde{I}_{i}^{l}}{\cup} \Gamma_{l}(t)
$$

verifies

$$
\left|\Sigma_{i}^{l}\right| \leqslant C \varepsilon_{n}^{\alpha} \varepsilon_{n}^{\gamma}
$$


In one hand we have

$$
\begin{aligned}
\mid \sum_{i=1}^{N_{\alpha}} \sum_{l=1}^{3} \bar{\psi}_{i}^{l} \int_{\left[r_{i}^{l} ; s_{i}^{l}\right] \backslash \tilde{I}_{i}^{l}} \oint_{\Gamma_{l}(t)} & \left(i \varphi_{n}, \nabla_{\tilde{A}_{n}} \varphi_{n}\right) \mid \\
& \leqslant C\|\psi\|_{\infty} \sum_{i=1}^{N_{\alpha}}\left|\Sigma_{i}^{l}\right|^{1 / 2}\left(\int_{\partial R_{i}}\left|\nabla_{\tilde{A}_{n}} \varphi_{n}\right|^{2}\right)^{1 / 2}(5.75) \\
& \leqslant C\|\psi\|_{\infty} \varepsilon_{n}^{\frac{\gamma-\alpha}{2}}\left(\log \frac{1}{\varepsilon_{n}}\right)^{1 / 2} \underset{n \rightarrow \infty}{\longrightarrow} 0
\end{aligned}
$$

On the other hand

$$
\begin{gathered}
\left|\sum_{i=1}^{N_{\alpha}} \sum_{l=1}^{3} \bar{\psi}_{i}^{l} \int_{\tilde{I}_{i}^{l}} \oint_{\Gamma_{l}(t)}\left(1-\frac{1}{\left|\varphi_{n}\right|^{2}}\right)\left(i \varphi_{n}, \nabla_{\tilde{A}_{n}} \varphi_{n}\right)\right| \\
\leqslant\left. C\|\psi\|_{\infty} \sum_{i=1}^{\mathcal{N}_{\alpha}} \int_{\partial R_{i}}|1-| \varphi_{n}\right|^{2}|| \nabla_{\tilde{A}_{n}} \varphi_{n} \mid \\
\leqslant C\|\psi\|_{\infty} \frac{1}{\varepsilon_{n}^{\alpha}} \varepsilon_{n} \log \frac{1}{\varepsilon_{n}} \longrightarrow 0 \quad \text { as } n \rightarrow \infty .
\end{gathered}
$$

Thus we are led to study the following quantities

$$
\int_{\tilde{I}_{i}^{l}} \oint_{\Gamma_{l}(t)}\left(i \frac{\varphi_{n}}{\left|\varphi_{n}\right|}, \nabla_{\tilde{A}_{n}} \frac{\varphi_{n}}{\left|\varphi_{n}\right|}\right)=\int_{\tilde{I}_{i}^{l}} 2 \pi d_{l}(t)-\int_{\tilde{I}_{i}^{l}} \oint_{\Gamma_{l}(t)} \tilde{A}_{n},
$$

where $d_{l}(t)$ is the degree of $\varphi /|\varphi|$ on $\Gamma_{l}(t)$ in any trivialisation of $E$ over $R_{i}$. Let $S_{i}^{l}(t)$ be the surface in $\partial R_{i}$ which has $\Gamma_{l}(t)$ as boundary (taking into account the orientation of $\left.\Gamma_{l}(t)\right)$, we have

$$
\int_{\tilde{I}_{i}^{l}} \oint_{\Gamma_{l}(t)} \tilde{A}_{n}=\int_{\tilde{I}_{i}^{l}} \int_{S_{i}^{l}(t)} * \tilde{h}_{n} \cdot \nu
$$

Moreover

$$
\left|\sum_{i=1}^{N_{\alpha}} \sum_{l=1}^{3} \bar{\psi}_{i}^{l} \int_{S_{i}^{l}(t)} * \tilde{h}_{n} . \nu\right| \leqslant C\|\psi\|_{\infty} \sum_{i=1}^{N_{\alpha}} \varepsilon_{n}^{\alpha} \int_{\partial R_{i}}\left|h_{0}\right|+\left|h_{n}\right| .
$$

By Lemma 2.3 we have

$$
\begin{aligned}
\sum_{i=1}^{N_{\alpha}} \varepsilon_{n}^{\alpha} \int_{\partial R_{i}}\left|h_{n}\right| & \leqslant \sum_{i=1}^{N_{\alpha}} \varepsilon_{n}^{\left(2+\frac{1}{2}\right) \alpha}\left(\log \frac{1}{\varepsilon_{n}}\right)^{1 / 2} \\
& \leqslant C \varepsilon_{n}^{\left(1+\frac{1}{2}\right) \alpha}\left(\log \frac{1}{\varepsilon_{n}}\right)^{1 / 2} \longrightarrow 0 \text { as } n \rightarrow+\infty .
\end{aligned}
$$


On $\partial R_{i}$ we use the bound $\left\|h_{0}\right\|_{L^{\infty}\left(\partial R_{i}\right)} \leqslant \frac{C}{\operatorname{dist}\left(\partial R_{i} ;\left\{x_{1}, \ldots, x_{n}\right\}\right)^{2}}$. Thus, using notations of Lemma 4.1, we have

$$
\begin{aligned}
\sum_{i=1}^{N_{\alpha}} \varepsilon_{n}^{\alpha} \int_{\partial R_{i}}\left|h_{0}\right| & \leqslant C \varepsilon_{n}^{3 \alpha} \sum_{i=1}^{N_{\alpha}} \frac{1}{\left|\operatorname{dist}\left(\partial R_{i} ;\left\{x_{1}, \ldots, x_{n}\right\}\right)\right|^{2}} \\
& \leqslant C \varepsilon_{n}^{3 \alpha} \sum_{j \in I_{\alpha}} \frac{1}{\left|\operatorname{dist}\left(z_{j} ;\left\{x_{1}, \ldots, x_{n}\right\}\right)\right|^{2}}
\end{aligned}
$$

where $I_{\alpha} \subset \varepsilon^{\alpha} \mathbb{Z}^{3}$ and $z_{j}$ are defined in part 4 .

We have

$$
\forall j \in I_{\alpha} \quad\left|\operatorname{dist}\left(z_{j},\left\{x_{1}, \ldots, x_{n}\right\}\right)\right| \geqslant \varepsilon_{n}^{\alpha}
$$

Let $2 \alpha / 3<\alpha^{\prime}<\alpha$. The number of elements of $\varepsilon_{n}^{\alpha} \mathbb{Z}^{3}$ included in $\cup_{k=1}^{n} C\left(\varepsilon_{n}^{\alpha^{\prime}}, x_{k}\right)$ is bounded by $C \varepsilon_{n}^{3\left(\alpha^{\prime}-\alpha\right)}$ thus separating the $j \in I_{\alpha}$ which can be included in $\cup_{k=1}^{n} \subset\left(\varepsilon_{n}^{\alpha^{\prime}}, x_{k}\right)$ and the ones in the exterior of this set we have

$$
\begin{aligned}
\sum_{i=1}^{N_{\alpha}} \varepsilon_{n}^{\alpha} \int_{\partial R_{i}}\left|h_{0}\right| & \leqslant C \varepsilon_{n}^{3 \alpha} \times \frac{1}{\varepsilon_{n}^{3\left(\alpha^{\prime}-\alpha\right)}} \times \frac{1}{\varepsilon_{n}^{2 \alpha}}+C \varepsilon_{n}^{3 \alpha} \times \frac{1}{\varepsilon_{n}^{3}} \times \frac{1}{\varepsilon_{n}^{2 \alpha^{\prime}}} \\
& \leqslant C \varepsilon_{n}^{3 \alpha^{\prime}-2 \alpha}+C \varepsilon_{n}^{2\left(\alpha-\alpha^{\prime}\right)}
\end{aligned}
$$

Since we have chosen $\alpha^{\prime}$ such that $3 \alpha^{\prime}-2>0$ and $\alpha^{\prime}<\alpha$ the right hand side of (5. 81) tends to zero. Combining the previous remark and (5. 80) we have

$$
\left|\sum_{i=1}^{N_{\alpha}} \sum_{l=1}^{3} \psi_{i}^{l} \int_{S_{i}^{l}(t)} * \tilde{h}_{n} \cdot \nu\right| \longrightarrow 0 \quad \text { as } \quad n \rightarrow 0
$$

Let us compute $\int_{\tilde{I}_{i}^{l}} 2 \pi d_{l}(t)$. First extend $d_{l}(t)$ on $\left[r_{i}^{l} ; s_{i}^{l}\right] \backslash \cup_{k=1}^{n_{i}}\left\{a_{i}^{k, l}\right\}$ in the following way : we extend $\varphi /|\varphi|$ continuously in a map from $\partial R_{i} \backslash \cup\left\{a_{i}^{k}\right\}$ into $S^{1}$ and for $t \in\left[r_{i}^{l} ; s_{i}^{l}\right] \backslash \cup_{k=1}^{n_{i}}\left\{a_{i}^{k, l}\right\} \cup \tilde{I}_{i}^{l}$ define $d_{l}(t)$ to be the degree of such an extension on $\Gamma_{l}(t)$. We clearly have

$$
\begin{aligned}
&\left|\sum_{i=1}^{N_{\alpha}} \sum_{l=1}^{n} \bar{\psi}_{i}^{l} \int_{\left[r_{i}^{l} ; s_{i}^{l}\right] \backslash \tilde{I}_{i}^{l}} 2 \pi d_{l}(t)\right| \leqslant C\|\psi\|_{\infty} \sum_{i=1}^{N_{\alpha}} C \varepsilon_{n}^{\gamma} \\
& \leqslant C\|\psi\|_{\infty} \varepsilon_{n}^{\gamma-\alpha} \longrightarrow 0 \text { as } n \rightarrow \infty \text {. } \\
& \text { Esaim : Cocv June 1996, Vol. } 1, \text { Pr. } 77-167
\end{aligned}
$$


For $l$ fixed, suppose we have chosen an indexation such that $a_{i}^{k, l}$ be increasing in $k$, we have

$$
\begin{aligned}
2 \pi \int_{x\left[r_{i}^{l} ; s_{i}^{l}\right]} d_{l}(t) & =2 \pi\left(a_{i}^{2, l}-a_{i}^{1, l}\right) \times\left(-d_{i}^{1, l}\right) \\
& +2 \pi\left(a_{i}^{3, l}-a_{i}^{2, l}\right) \times\left(-d_{i}^{1, l}-d_{i}^{2, l}\right)+\ldots \\
& +2 \pi\left(a_{i}^{n_{i}, l}-a_{i}^{n_{i}, l}\right) \times\left(-d_{i}^{1, l}-\ldots-d_{i}^{n_{i}-1, l}\right) \\
& +2 \pi\left(s_{i}^{l}-a_{i}^{n_{i}, l}\right) \times\left(-\sum_{k=1}^{n_{i}} d_{i}^{k, l}\right) .
\end{aligned}
$$

Considering (5. 50) we get

$$
2 \pi \int_{r_{i}^{l}}^{s_{i}^{l}} d_{l}(t)=2 \pi \sum_{k=1}^{n_{i}} d_{i}^{k, l} a_{i}^{k, l} .
$$

On the other hand we have

$$
<\mathbb{L}_{i}^{\varepsilon_{n}} ; e_{l}>=\sum_{k=1}^{n_{i}} d_{i}^{k, l} a_{i}^{k, l}
$$

Thus

$$
\sum_{i=1}^{N_{\alpha}} \sum_{l=1}^{3} \bar{\psi}_{i}^{l} \quad 2 \pi \int_{r_{i}^{l}}^{s_{i}^{l}} d_{l}(t)=\sum_{i=1}^{N_{\alpha}}<\mathbb{L}_{i}^{\varepsilon_{n}} ; \bar{\psi}_{i}>
$$

Moreover

$$
\begin{aligned}
<\mathbb{L}^{\varepsilon_{n}} ; \psi> & =\sum_{i=1}^{N_{\alpha}}\left\langle\mathbb{L}_{i}^{\varepsilon_{n}} ; \psi>\right. \\
& =\sum_{i=1}^{N_{\alpha}}<\mathbb{L}_{i}^{\varepsilon_{n}} ; \bar{\psi}_{i}>+\sum_{i=1}^{N_{\alpha}}<\mathbb{L}_{i}^{\varepsilon} ; \psi-\bar{\psi}_{i}>,
\end{aligned}
$$

and we have

$$
\begin{aligned}
\left|\sum_{i=1}^{N_{\alpha}}<\mathbb{L}_{i}^{\varepsilon_{n}}, \psi-\bar{\psi}_{i}>\right| & \leqslant \sum_{i=1}^{N_{\alpha}} M\left(\mathbb{L}_{i}^{\varepsilon_{n}}\right)\|\nabla \psi\|_{\infty} \varepsilon_{n}^{\alpha} \\
& \leqslant M\left(\mathbb{L}_{1}\right)\|\nabla \psi\|_{\infty} \varepsilon_{n}^{\alpha} \longrightarrow 0 \text { as } n \rightarrow 0
\end{aligned}
$$

Combining (5. 69), (5. 71), (5. 72), (5. 73), (5. 75), (5. 76), (5. 82), (5. $83),(5.87)$ and $(5.88)$ we get

$$
-\int_{\partial T_{\varepsilon_{n}^{\alpha}}} * \psi \cdot d^{*} h_{n} \wedge \nu+<\mathbb{L}^{\varepsilon_{n}} ; \psi>\longrightarrow 0 \quad \text { as } \quad n \rightarrow+\infty
$$

Combining (5. 65), (5. 66), (5. 67), (5. 68) and (5. 90) we prove (5. 63) which implies (5. 49).

It remains to prove that $\mathcal{H}^{1}(\operatorname{supp} \mathbb{L})<+\infty$. Let $r$ be a positive number, Esaim : CoCv June 1996, Vol.1, PP. 77-167 
(we take $r<\eta$ where $\eta$ is the constant given by Lemma 3.1). Consider the lattice $r \mathbb{Z}^{3}$, as in the proof of Lemma 4.1 the number of $x \in r \mathbb{Z}^{3}$ such that

$$
\frac{1}{2 r} \int_{\partial C(2 r, x)}\left|\nabla_{\tilde{A}_{n}} \varphi_{n}\right|^{2}+\frac{1}{2 \varepsilon_{n}^{2}}\left(1-\left|\varphi_{n}\right|^{2}\right)^{2}+\left|h_{n}\right|^{2} \geqslant \eta \log \left(\frac{2 r}{\varepsilon_{n}}\right)
$$

is bounded by $C / r$. This implies that $\operatorname{supp}\left(\mathbb{L}^{\varepsilon_{n}}\right)$ can be included in $\frac{C}{r}$ cubes of edge $2 r$. Let $R>0$, we can extract from $\varepsilon_{n}$ a subsequence such that the bad cubes $C(2 r, z)$ for $z \in r \mathbb{Z}^{3} \cap B_{R}(0)$ are always the same, thus we are sure that supp $\mathbb{L} \cap B_{R}(0)$ is included in those cubes. Thus $\forall r, R>$ 0 supp $\mathbb{L} \cap B_{R}(0)$ is included in $C / r$ cubes where $C$ is independent of $R$ and $r$, this implies

$$
\mathcal{H}^{1}(\operatorname{supp} \mathbb{L})<+\infty
$$

\section{Strong Convergence of The Magnetic Field AWAY From THE LIMITING SINGULAR SET}

In this part we prove the strong convergence in $H_{l o c}^{1}\left(\mathbb{R}^{3} \backslash\right.$ supp $\left.\mathbb{L}\right)$ of the magnetic field $h_{\varepsilon_{n}}$ for the subsequence given by theorem 5.1. As in [2] and [3], with some more work, it should be possible to prove the convergence away from the limiting singular set in a stronger norm, $C^{k}(k \geqslant 0)$ for instance.

Theorem 6.1. Let $h_{\varepsilon_{n}}$ be the sequence given by theorem 5.1 and let $\mathbb{L}$ be the rectifiable current in the same theorem. We have

$$
h_{\varepsilon_{n}} \rightarrow h_{\star} \text { strongly in } H_{l o c}^{1}\left(\mathbb{R}^{3} \backslash \text { supp } \mathbb{L}\right) \text {. }
$$

\section{Proof of theorem 6.1.}

We still use the notation $A_{n}, h_{n}, \varphi_{n} \ldots$ for $A_{\varepsilon_{n}}, h_{\varepsilon_{n}}, \varphi_{\varepsilon_{n}} \ldots$ Let $x_{0} \in$ $\mathbb{R}^{3} \backslash$ supp $\mathbb{L}$ and $r$ small enough such that $B_{r}\left(x_{0}\right) \subset U_{l}$ for some $l \in$ $\{1, \ldots, k+1\}$ (we use the notation of part 3 ) and such that $r<\min \left(\eta ; d_{x_{0}} / \eta\right)$ where $\eta$ is given by Lemma 3.1. This is possible since $\left\{x_{1}, \ldots, x_{n}\right\} \subset$ supp $\mathbb{L}$. Remark that we have $B_{r}\left(x_{0}\right) \cap \sup p \mathbb{L}=\emptyset$.

We will prove that $h_{n}$ converges strongly in $H^{1}\left(B_{r / 4}\left(x_{0}\right)\right)$ to $h_{\star}$ given by $(5$. $49)$.

Since the limit $h_{\star}$ is well identified (= is unique) and since we have weak convergence in $W^{1, p}(1<p<3 / 2) h_{n} \rightarrow h_{\star}$, it is sufficient to prove the existence of one subsequence of $h_{n}$ which strongly converges in $H^{1}$ to prove the strong convergence of the complete sequence.

We can trivialize $E$ over $B_{r}\left(x_{0}\right)$ and since $B_{r}\left(x_{0}\right)$ is included in some $U_{l}$ we can take the Coulomb gauge given by proposition 2.1 ie $\tilde{A}=A_{l}^{0}+A_{n}$. Since $h_{n}$ weakly converges in $W^{1, p}\left(B_{r}\left(x_{0}\right)\right), A_{n}$ strongly converges in $W^{1, p}\left(B_{r}\left(x_{0}\right)\right)$ and because of corollary $5.1(5.38)$ we can extract a subsequence such that $\varphi_{n}$ weakly converges in $W^{1, p}\left(B_{r}\left(x_{0}\right)\right)(1<p<3 / 2)$ [in fact it is not difficult to see that the complete sequence $\varphi_{n}$ converges but, because of the 
remark above, we do not take care of it]. Let $\varphi_{\star}$ be the limit of $\varphi_{n}$, since $\int_{B_{r}\left(x_{0}\right)}\left(1-\left|\varphi_{n}\right|^{2}\right)^{2} \rightarrow 0,\left|\varphi_{\star}\right| \equiv 1$ and passing to the limit in (2. 15) we have

$$
-d^{\star} h_{\star}=\left(i \varphi_{\star}, d \varphi_{\star}\right)-A_{l}^{0}-A_{\star} .
$$

Moreover since supp $\mathbb{L} \cap B_{r}\left(x_{0}\right)=\emptyset,(5.49)$ implies

$$
-d d^{\star} h_{\star}+d A_{\star}+d A^{0}=0 \quad \text { in } \mathcal{D}^{\prime}\left(B_{r}\left(x_{0}\right)\right) \text {. }
$$

Combining (6.2) and (6.3) we get

$$
d\left(i \varphi_{\star}, d \varphi_{\star}\right)=0 \quad \text { in } \quad \mathcal{D}^{\prime}\left(B_{r}\left(x_{0}\right)\right) \quad .
$$

Moreover since $d^{*} A_{l}^{0}=0$ and $d^{*} A_{\star}=0$ in $\left(B_{r}\left(x_{0}\right)\right)$ we have also

$$
d^{*}\left(i \varphi_{\star}, d \varphi_{\star}\right)=0 \quad \text { in } \quad \mathcal{D}^{\prime}\left(B_{r}\left(x_{0}\right)\right) .
$$

From $(6.4)$ and $(6.5)$ we deduce the existence of an harmonic function $H$ such that

$$
\left(i \varphi_{\star}, d \varphi_{\star}\right)=d H
$$

Since $\left|\varphi_{\star}\right| \equiv 1$, we have

$$
d \varphi_{\star}=\left(i \varphi_{\star}, d \varphi_{\star}\right) i \varphi_{\star}
$$

Combining (6.6) and (6.7) we deduce that

$$
d\left(\varphi_{\star} e^{-i H}\right)=0 \quad \text { in } \quad \mathcal{D}^{\prime}\left(B_{r}\left(x_{0}\right)\right)
$$

Thus $\varphi_{\star}$ is a harmonic map from $B_{r}\left(x_{0}\right)$ into $S^{1}$, in particular $\varphi_{\star} \in C^{\infty}\left(B_{r}\left(x_{0}\right)\right)$.

Our aim, now, is to prove that there exists a subsequence, still denoted $\varphi_{n}$ such that for $n$ sufficiently large we have

$$
\left|\varphi_{n}\right| \geqslant \frac{1}{2} \quad \text { in } \quad B_{r / 4}\left(x_{0}\right) \text {. }
$$

Indeed, as we have already seen below, (6.9) and the weak convergence of $h_{n}$ imply the strong convergence in $B_{r / 4}\left(x_{0}\right)$.

Let $0<s<r, s$ will be chosen sufficiently small later. Consider $s \mathbb{Z}^{3} \cap$ $B_{r}\left(x_{0}\right)$ as in the end of the proof of theorem V.1 $B_{r}\left(x_{0}\right) \cap s u p p \mathbb{L}^{\varepsilon_{n}}$ is included in $C / s$ cubes $C(2 s, x)$ where $x \in s \mathbb{Z}^{3}$ where $C$ does not depend neither on $s$ nor on $\varepsilon_{n}$. Extract a subsequence such that the bad cubes in $B_{r}\left(x_{0}\right)$ are always the same and denote by $T_{s}$ this union of cubes.

Let $\Omega_{r, s}=B_{r}\left(x_{0}\right) \backslash \bar{T}_{s}$ we claim that $h_{n} \rightarrow h_{\star}$ strongly in $H_{l o c}^{1}\left(\Omega_{r, s}\right)$. Let $\omega$ be an open set such that $\omega \subset \subset \Omega_{r, s}$ and $\chi$ be a non negative cut-off function such that

$$
\begin{cases}\chi \equiv 1 & \text { in } \omega \\ \chi \equiv 0 & \text { in } \mathbb{R}^{3} \backslash \Omega_{r, s} .\end{cases}
$$

From (2. 15) and the fact that $\left|\varphi_{n}\right| \geqslant \frac{1}{2}$ in $\Omega_{r, s}$ we have

$$
-d\left(\frac{1}{\left|\varphi_{n}\right|^{2}} d^{*} \tilde{h}_{n}\right)+\tilde{h}_{n}=0 \quad \text { in } \quad \Omega_{r, s} .
$$


Let $K_{n}$ be the solution of

$$
\left\{\begin{array}{lll}
-\Delta K_{n}+K_{n}=f_{n} & \text { in } & \Omega_{r, s} \\
K_{n}=0 & \text { on } & \partial \Omega_{r, s}
\end{array},\right.
$$

where $f_{n}=d^{\star} \tilde{h}_{n} /\left|d^{\star} \tilde{h}_{n}\right|^{\beta}$ in $\omega$ and $f_{n} \equiv 0$ in $\Omega_{r, s} \backslash \omega$ for $0<\beta<1$ (fixed later). Let $k_{n}=d K_{n}$. For $q$ and $\beta$ such that $q(1-\beta)<2$ and $2<q<3$ we have by classical elliptic estimates

$$
\left(\int_{\Omega_{r, s}}\left|k_{n}\right|^{\frac{3 q}{3-q}}\right)^{\frac{1}{q}-\frac{1}{3}}+\left(\int_{\Omega_{r, s}}\left|d^{\star} k_{n}\right|^{q}\right)^{1 / q} \leqslant C\left(\int_{\omega}\left|d^{*} \tilde{h}_{n}\right|^{q(1-\beta)}\right)^{1 / q}
$$

Multiplying (6.11) by $\chi k_{n}$ and integrating on $\Omega_{r, s}$ we have

$$
\begin{aligned}
& \int_{\Omega_{r, s}} \chi d^{*} k_{n} d^{*} \tilde{h}_{n}+\int_{\Omega_{r, s}} k_{n} \cdot d^{*} \tilde{h}_{n} \wedge d \chi+\int_{\Omega_{r, s}} \chi k_{n} \tilde{h}_{n} \\
& =\int_{\Omega_{r, s}}\left(1-\frac{1}{\left|\varphi_{n}\right|^{2}}\right)\left[d^{*} \tilde{h}_{n} d^{*} k_{n} \chi+k_{n} \cdot d^{*} \tilde{h}_{n} \wedge \chi\right] .
\end{aligned}
$$

In the other hand, taking the exterior derivative of (6.12), multiplying by $\chi \tilde{h}_{n}$ and integrating on $\Omega_{r, s}$ we have

$$
\begin{aligned}
\int_{\Omega_{r, s}} \chi d^{*} \tilde{h}_{n} \cdot d^{*} k_{n} & +\chi \tilde{h}_{n} \cdot k_{n}+\tilde{h}_{n} \cdot d \chi \wedge d^{*} k_{n} \\
& =-\int_{\omega}\left|d^{*} \tilde{h}_{n}\right|^{2-\beta}
\end{aligned}
$$

Combining (6.14) and (6.15) we get

$$
\begin{aligned}
\int_{\omega}\left|d^{*} \tilde{h}_{n}\right|^{2-\beta} \leqslant & C\left[\int_{\Omega_{r, s}}\left(1-\left|\varphi_{n}\right|^{2}\right)\left|d^{*} \tilde{h}_{n}\right|\left|d^{*} k_{n}\right|+\right. \\
& \left.+\int_{\Omega_{r, s}}\left|k_{n}\right|\left|d^{*} \tilde{h}_{n}\right|+\left|\tilde{h}_{n}\right|\left|d^{*} k_{n}\right|\right]
\end{aligned}
$$

First of all we have

$$
\begin{aligned}
& \left|\int_{\Omega_{r, s}}\left(1-\left|\varphi_{n}\right|^{2}\right)\right| d^{*} \tilde{h}_{n}\left|\left(\left|d^{*} k_{n}\right|+\left|k_{n}\right|\right)\right| \leqslant \\
& \leqslant C \varepsilon_{n}^{2\left(1-\frac{1}{2}-\frac{1}{q}\right)}\left(\log \frac{1}{\varepsilon_{n}}\right)^{1 / 2}\left[\int_{\omega}\left|d^{*} \tilde{h}_{n}\right|^{q(1-\beta)}\right]^{1 / q} .
\end{aligned}
$$

we have also

$$
\int_{\Omega_{r, s}}\left|k_{n}\right|\left|d^{*} \tilde{h}_{n}\right| \leqslant\left(\int_{\Omega_{r, s}}\left|k_{n}\right|^{\frac{3 q}{3-q}}\right)^{\frac{1}{q}-\frac{1}{3}} \times\left(\int_{\Omega_{r, s}}\left|d^{*} \tilde{h}_{n}\right|^{\frac{3 q}{4 q-3}}\right)^{1+\frac{1}{3}-\frac{1}{q}}(6.18)
$$


and for $2<q<3, \frac{2}{3}<\frac{5}{6}<\frac{4}{3}-\frac{1}{q}<1$, thus (6. 18) implies

$$
\int_{\Omega_{r, s}}\left|k_{n}\right|\left|d^{*} \tilde{h}_{n}\right| \leqslant C\left(\int_{\omega}\left|d^{*} \tilde{h}_{n}\right|^{q(1-\beta)}\right)^{1 / q} .
$$

Finally

$$
\int_{\Omega_{r, s}}\left|\tilde{h}_{n}\right|\left|d^{*} k_{n}\right| \leqslant\left(\int_{\Omega_{r, s}}\left|\tilde{h}_{n}\right|^{1-\frac{1}{q}}\right)\left(\int_{\Omega_{r, s}}\left|d^{*} k_{n}\right|^{q}\right)^{1 / q}
$$

and since $\tilde{h}_{n}$ is bounded in $W^{1, p}\left(B_{r}\left(x_{0}\right)\right)$ for any $1<p<\frac{3}{2}, \tilde{h}_{n}$ is bounded in $L^{s}\left(B_{r}\left(x_{0}\right)\right)$ for any $1<s<3$ and since $2<q, \frac{q}{q-1}<2$ and (6. 20) combined with $(6.13)$ gives

$$
\int_{\Omega_{r, s}}\left|\tilde{h}_{n}\right|\left|d^{*} k_{n}\right| \leqslant C\left(\int_{\omega}\left|d^{*} \tilde{h}_{n}\right|^{q(1-\beta)}\right)^{1 / q} .
$$

Combining $(6.16),(6.17),(6.19)$ and $(6.20)$ we have

$$
\int_{\omega}\left|d^{*} \tilde{h}_{n}\right|^{2-\beta} \leqslant C\left(\int_{\omega}\left|d^{*} \tilde{h}_{n}\right|^{q(1-\beta)}\right)^{1 / q} .
$$

Taking $2-\beta=q(1-\beta), 2<q<3$ implies $\frac{3}{2}<2-\beta<2$ thus we have

$$
\forall 1 \leqslant p<2 \quad \int_{\omega}\left|d^{*} \tilde{h}_{n}\right|^{p}<C \quad \text { indep. of } n
$$

Let $\omega^{\prime} \subset \subset \omega$ since we have

$$
\Delta \tilde{h}_{n}=d\left(d^{*} \tilde{h}_{n}\right) \quad \text { in } \omega
$$

and since $\tilde{h}_{n}$ is uniformly bounded in $W^{1, p}(\omega) 1 \leqslant p<\frac{3}{2}$ in view of $(6$. 22 ) we have, by classical elliptic estimates that $\tilde{h}_{n}$ is uniformly bounded in $W^{1, p}\left(\omega^{\prime}\right) 1 \leqslant p<2$.

Thus we have proved that $\tilde{h}_{n}$ is uniformly bounded in any compact set included in $\Omega_{r, s}$ for the norm $W^{1, p} 1 \leqslant p<2$.

We establish now the strong $H_{l o c}^{1}$ convergence of $\tilde{h}_{n}$ in $\Omega_{r, s}$. Multiplying (6.11) by $\chi \tilde{h}_{n}$ and integrating on $\Omega_{r, s}$ we have

$$
\int_{\Omega_{r, s}} \chi \frac{1}{\left|\varphi_{n}\right|^{2}}\left|d^{*} \tilde{h}_{n}\right|^{2}+\int_{\Omega_{r, s}} \frac{1}{\left|\varphi_{n}\right|^{2}} \tilde{h}_{n} . d \chi \wedge d^{*} \tilde{h}_{n}+\chi\left|\tilde{h}_{n}\right|^{2}=0 .
$$

Thus we have

$$
\int_{\omega}\left|d^{*} \tilde{h}_{n}\right|^{2} \leqslant C\left(\int_{\text {supp }}\left|\tilde{h}_{n}\right|^{4}\right)^{1 / 4}\left(\int_{\Omega_{r, s}}\left|d^{*} \tilde{h}_{n}\right|^{\frac{4}{3}}\right)^{3 / 4} .
$$

Since $\tilde{h}_{n}$ is uniformly bounded for the norm $W^{1, p}$ for any $p<2$ on supp $\chi \subset \subset$ $\Omega_{r, s}, \tilde{h}_{n}$ converges to $h_{\star}$ in $L^{4}(\operatorname{supp} \chi)$ and we obtain that $d^{*} \tilde{h}_{n}$ is uniformly bounded for the $L^{2}$ norm on any compact set included in $\Omega_{r, s}$. 
Multiplying (6.11) by $\chi\left(\tilde{h}_{n}-\tilde{h}_{\star}\right)$, we have

$$
\begin{aligned}
\int_{\Omega_{r, s}} \chi \frac{1}{\left|\varphi_{n}\right|^{2}} & \left|d^{*} \tilde{h}_{n}\right|^{2}-\int_{\Omega_{r, s}} \chi \frac{1}{\left|\varphi_{n}\right|^{2}} d^{*} \tilde{h}_{\star} \cdot d^{*} \tilde{h}_{n}+ \\
& +\int_{\Omega_{r, s}} \frac{1}{\left|\varphi_{n}\right|^{2}}\left(\tilde{h}_{n}-\tilde{h}_{\star}\right) \cdot d \chi \wedge d^{*} \tilde{h}_{n} \int_{\Omega_{r, s}} \chi\left(\tilde{h}_{n}-\tilde{h}_{\star}\right) \cdot \tilde{h}_{n}=0
\end{aligned}
$$

Hence we deduce that

$$
\int_{\Omega_{r, s}} \chi \frac{1}{\left|\varphi_{n}\right|^{2}}\left|d^{*} \tilde{h}_{n}\right|^{2} \longrightarrow \int_{\Omega_{r, s}} \chi\left|d^{*} \tilde{h}_{\star}\right|^{2} \quad \text { as } n \rightarrow \infty \quad .
$$

By lower semicontinuity this implies the strong convergence of $d^{*} \tilde{h}_{n}$ to $d^{*} \tilde{h}_{\star}$ in $L^{2}(K)$ for any compact $K$ included in $\Omega_{r, s}$. Because of $(6.23)$, since we have already strong convergence of $\tilde{h}_{n}$ to $h_{\star}$ in $L^{2}\left(\Omega_{r, s}\right)$ we deduce that $\tilde{h}_{n}$ strongly converges to $h_{\star}$ in $H_{l o c}^{1}\left(\Omega_{r, s}\right)$. This convergence, combined with $(2$. 15), yields

$$
\left(i \frac{\varphi_{n}}{\left|\varphi_{n}\right|}, d \frac{\varphi_{n}}{\left|\varphi_{n}\right|}\right) \longrightarrow\left(i \varphi_{\star}, d \varphi_{\star}\right) \quad \text { in } \quad L_{l o c}^{2}\left(\Omega_{r, s}\right)
$$

Moreover, since $\varphi_{\star} \in C^{\infty}\left(B_{r}\left(x_{0}\right), S^{1}\right)$, the degree of $\varphi_{\star}$ on all the generators of $\pi_{1}\left(\Omega_{r, s}\right)$ is 0 and the convergence $(6.26)$ implies that this is also the case for $\varphi_{n} /\left|\varphi_{n}\right|$ for $n$ chosen sufficiently large. Let $\Gamma$ be one of the regular curves in $\Omega_{r, s}$ which generates $\pi_{1}\left(\Omega_{r, s}\right)$ and let $U$ be a tubular neighborhood of $\Gamma$ in $\Omega_{r, s}$. Consider a closed 2 -form $\omega_{\Gamma}$ with support in $U$ which is the Poincaré dual of $\Gamma$ (see [5] I.6 for the construction of $\omega_{\Gamma}$ ) that is, for any closed 1 -form $\psi$ in $U, \omega_{\Gamma}$ verifies

$$
\int_{U} \psi \wedge \omega_{\Gamma}=\int_{\Gamma} \psi
$$

Since $\varphi_{n} /\left|\varphi_{n}\right|$ is regular in $U$

$$
d\left(i \frac{\varphi_{n}}{\left|\varphi_{n}\right|}, d \frac{\varphi_{n}}{\left|\varphi_{n}\right|}\right)=0 \quad \text { in } \quad U
$$

Thus

$$
\int_{U}\left(\frac{i \varphi_{n}}{\left|\varphi_{n}\right|}, d \frac{\varphi_{n}}{\left|\varphi_{n}\right|}\right) \wedge \omega_{\Gamma}=\int_{\Gamma}\left(\frac{i \varphi_{n}}{\left|\varphi_{n}\right|}, d \frac{\varphi_{n}}{\left|\varphi_{n}\right|}\right)=2 \pi \operatorname{deg} \frac{\varphi_{n}}{\left|\varphi_{n}\right|}
$$

and in the same way

$$
\int_{U}\left(i \varphi_{\star}, d \varphi_{\star}\right) \wedge \omega_{\Gamma}=\int_{\Gamma}\left(i \varphi_{\star}, d \varphi_{\star}\right)=2 \pi \operatorname{deg} g_{\Gamma} \varphi_{\star}=0
$$

Since

$$
\int_{U}\left(\frac{i \varphi_{n}}{\left|\varphi_{n}\right|}, d \frac{\varphi_{n}}{\left|\varphi_{n}\right|}\right) \wedge \omega_{\Gamma}=\int_{\Gamma}\left(i \varphi_{\star}, d \varphi_{\star}\right) \wedge \omega_{\Gamma}
$$

we have, for $n$ chosen sufficiently large, $\operatorname{deg} \frac{\varphi_{n}}{\left|\varphi_{n}\right|}=0$.

We prove now that $\left|\varphi_{n}\right| \geqslant \frac{1}{2}$ in $B_{r / 4}\left(x_{0}\right)$ that is $T_{r, s} \cap B_{r / 4}\left(x_{0}\right)=\emptyset$. Since we have already established the strong convergence in $H_{l o c}^{1}\left(B_{r}\left(x_{0}\right) \backslash T_{r, s}\right)$ of 
$\tilde{h}_{n}$, in view of Lemma 3.1, to get the desired result, it suffices to prove that, for $n$ sufficiently large, we have

$$
\frac{2}{r} \int_{B_{r / 2}\left(x_{0}\right)}\left|\nabla_{\tilde{A}} \varphi_{n}\right|^{2}+\frac{1}{2 \varepsilon_{n}^{2}}\left(1-\left|\varphi_{n}\right|^{2}\right)^{2}+\left|h_{n}\right|^{2}<\eta \log \left(\frac{r}{2 \varepsilon_{n}}\right) .
$$

Applying the mean value formula we deduce the existence of $\rho \in\left(\frac{r}{2} ; r\right)$ such that

$$
\left\{\begin{array}{l}
\partial B_{\rho}\left(x_{0}\right) \cap T_{r ; s} \subset N \text { balls of radius } s \\
\text { denoted } \cup_{i=1}^{N} B\left(a_{i}, s\right) \text { where } N \text { is indep. of } s \text { and } \varepsilon_{n} . \\
\int_{\partial B_{\rho}\left(x_{0}\right)}\left|\nabla_{\tilde{A}_{n}} \varphi_{n}\right|^{2}+\frac{1}{2 \varepsilon_{n}^{2}}\left(1-\left|\varphi_{n}\right|^{2}\right)^{2}+\left|h_{n}\right|^{2} \leqslant C \log \left(\frac{1}{\varepsilon_{n}}\right) \\
\int_{\partial B_{\rho}\left(x_{0}\right) \backslash \cup_{i=1}^{N} B\left(a_{i}, 2 s\right)}\left|\nabla \varphi_{n}\right|^{2} \leqslant C(r, s)
\end{array}\right.
$$

This is possible because $T_{r, s}$ is included in $C / s$ balls of radius $s$ (where $C$ is independent of $s$ and $\varepsilon_{n}$ ), because Lemma 2.5 holds and $\varphi_{n}$ converges strongly in $H_{l o c}^{1}\left(T_{r, s}\right)$. First of all, since $N$ is independent on $s$ and $\varepsilon_{n}$, there exists $\lambda \geqslant 2$ (independent on $s$ and $\varepsilon_{n}$ ) $N^{\prime} \leqslant N$ and $\left(b_{j}\right)_{j \in N^{\prime}}$ such that

$$
\left\{\begin{array}{l}
b_{j} \in \partial B_{\rho}\left(x_{0}\right) \\
\cup_{i=1}^{N} B\left(a_{i}, s\right) \subset \cup_{j=1}^{N^{\prime}} B\left(b_{j}, \lambda s\right) \\
\text { for } j \neq j^{\prime} \quad B\left(b_{j}, 2 \lambda s\right) \cap B\left(b_{j^{\prime}}, 2 \lambda s\right)=\emptyset
\end{array}\right.
$$

To prove $(6.32)$, since $\left(\varphi_{n}, \tilde{A}_{n}\right)$ is a minimizer of the energy, it suffices to find $\varphi_{n}^{\prime} \quad B_{\rho}\left(x_{0}\right) \longrightarrow \mathbb{C}$ such that $\varphi_{n}^{\prime}=\varphi_{n}$ on $\partial B_{\rho}\left(x_{0}\right)$ and such that $\left(\varphi_{n}^{\prime}, \tilde{A}_{n}\right)$ verifies $(6.32)$.

\section{Construction of $\varphi_{n}^{\prime}$.}

First, for $\rho \geqslant t \geqslant \rho-s$, we contract the bad parts contained in the $B\left(b_{j}, \lambda s\right)$ in the following way. By the mean value formula we can find $\mu \in(\lambda, 2 \lambda)$ such that

$$
\forall j \leqslant N^{\prime} \quad \int_{\partial\left(B\left(b_{j}, \mu s\right) \cap \partial B_{\rho}\left(x_{0}\right)\right)}\left|\nabla \varphi_{n}\right|^{2} \leqslant C(r, s)
$$

and

$$
\forall j \leqslant N^{\prime} \quad \int_{\partial\left(B\left(b_{j}, \mu s\right) \cap \partial B_{\rho}\left(x_{0}\right)\right)} \frac{1}{2 \varepsilon_{n}^{2}}\left(1-\left|\varphi_{n}\right|^{2}\right)^{2} \leqslant \frac{C}{s} \log \frac{1}{\varepsilon_{n}},
$$

where $C$ is independent of $n$ and $s$.

For $\rho-s \leqslant t \leqslant \rho$ denote by $T_{t}$ the following set

$$
T_{t}=\frac{t}{\rho}\left[\partial B_{\rho}\left(x_{0}\right) \backslash \cup_{j=1}^{N^{\prime}} B\left(b_{j}, \mu s\right)\right]
$$


and

$$
T=\underset{\rho-s \leqslant t \leqslant \rho}{\cup} T_{t}
$$

Let $B^{2}(x, l)$ be the geodesic ball in $\partial B\left(x_{0},\left|x-x_{0}\right|\right)$ of centre $x$ and radius $2 \arcsin (l / 2|x|)$ corresponding to the chord of length $l$.

In $B^{2}\left(\frac{t}{\rho} b_{j}, \frac{t}{\rho} \mu s\right)$ a point $x$ is represented by the coordinates $(\theta, l, t)$ where $\theta$ is the angle in geodesic polar coordinates in $B^{2}\left(\frac{t}{\rho} b_{j} ; \frac{t}{\rho} \mu s\right), l$ is the distance to $\frac{t}{\rho} b_{j}$ and $t=|x|$.

We have seen above that, for $n$ sufficiently large, the degree of $\varphi_{n} /\left|\varphi_{n}\right|$ on $\partial\left(B^{2}\left(b_{j}, \mu s\right) \cap B_{\rho}\left(x_{0}\right)\right)=\partial B^{2}\left(b_{j}, \mu s\right)$ is equal to 0 . Thus there exists a regular function $\psi_{n}^{j} \partial B^{2}\left(b_{j}, \mu s\right) \longrightarrow \mathbb{R}$ such that

$$
\left\{\begin{array}{l}
\frac{\varphi_{n}}{\left|\varphi_{n}\right|}(\theta, \mu s, \rho)=e^{i \psi_{n}^{j}(\theta)} e^{i \theta_{n}^{j}} \quad \text { in } \partial B^{2}\left(b_{j}, \mu s\right) \\
\text { and } \\
\int_{\partial B^{2}\left(b_{j}, \mu s\right)} \psi^{j}=0,
\end{array}\right.
$$

where $\theta_{n}^{j}$ is the representant, in $\left[0 ; 2 \pi\right.$ [ of the mean value of $\varphi_{n} /\left|\varphi_{n}\right|$ along $\partial B^{2}\left(b_{j}, \mu s\right)$. From $(6.35)$ and Poincaré inequality, we deduce that

$$
\int_{\partial B^{2}\left(b_{j}, \mu s\right)}\left|\psi_{n}^{j}\right|^{2} \leqslant C \int_{\partial B^{2}\left(b_{j}, \mu s\right)}\left|\nabla \psi_{n}^{j}\right|^{2} \leqslant C(s, r) .
$$

Let $C^{j}=\cup_{\rho-s<t<\rho} \frac{t}{\rho} B^{2}\left(b_{j}, \mu s\right)=\cup_{\rho-s<t<\rho} B^{2}\left(\frac{t}{\rho} b_{j}, \frac{t}{\rho} \mu s\right) . C^{j}$ is starshaped from $b_{j}^{s}=\left(1-\frac{s}{2 \rho}\right) b_{j}$. Denote by $\pi^{j}$ the map from $\bar{C}^{j}$ into $\partial C^{j}$ which associates to $x \in \bar{C}^{j}$ the point of $\partial C^{j}$ which is on the line starting from $b_{j}^{s}$ to $x$. We have

$$
\left|\nabla \pi^{j}\right|(x) \leqslant \frac{C s}{\left|x-b_{j}^{s}\right|}
$$

where $C$ is independent of $s$.

Define $\varphi_{n}^{\prime}(x)$ for $\rho-s \leqslant|x| \leqslant \rho$ in the following way

$$
\left\{\begin{array}{c}
\varphi_{n}^{\prime}(x)=\varphi_{n}\left(\frac{\rho x}{t}\right) \quad \text { in } \bar{T}_{t} \\
\varphi_{n}^{\prime}(\theta, l, \rho-s)=\left[1+\left(\left|\varphi_{n}\right|(\theta, \mu s ; \rho)-1\right) \frac{l}{\mu s} \frac{\rho}{\rho-s}\right] \times \\
\quad \times \exp \left[i \frac{l}{\mu s} \frac{\rho}{\rho-s} \psi_{n}^{j}(\theta, \mu s, \rho)\right] \times e^{i \theta_{n}^{j}} \\
\text { in } B^{2}\left(\frac{\rho-s}{\rho} b_{j}, \frac{\rho-s}{\rho} \mu s\right) \\
\text { and } \varphi_{n}^{\prime}(x)=\varphi_{n}^{\prime}\left(\pi^{j}(x)\right) \quad \text { in } C^{j}
\end{array}\right.
$$

First of all in view of $(6.33)$ we have

$$
\int_{T}\left|\nabla \varphi_{n}^{\prime}\right|^{2} \leqslant C \int_{\rho-s}^{\rho} \int_{T_{\rho}}\left|\nabla \varphi_{n}\right|^{2} \leqslant C(s, r)
$$


and

$$
\int_{T} \frac{1}{\varepsilon_{n}^{2}}\left(1-\left|\varphi_{n}^{\prime}\right|^{2}\right)^{2} \leqslant C \int_{\rho-s}^{\rho} \int_{T_{\rho}} \frac{1}{\varepsilon_{n}^{2}}\left(1-\left|\varphi_{n}\right|^{2}\right)^{2} \leqslant C s \log \frac{1}{\varepsilon_{n}}
$$

On $\partial T \cap \partial C_{j}$ we have also, in view of $(6.35)$

$$
\int_{\partial T \cap \partial C_{j}}\left|\nabla \varphi_{n}^{\prime}\right|^{2} \leqslant C \int_{\rho-s}^{\rho} \int_{\partial B^{2}\left(b_{j}, \mu s\right)}\left|\nabla \varphi_{n}\right|^{2} \leqslant C(s, r)
$$

and in view of $(6.36)$

$$
\begin{aligned}
\int_{\partial T \cap \partial C_{j}} \frac{1}{\varepsilon_{n}^{2}}\left(1-\left|\varphi_{n}^{\prime}\right|^{2}\right)^{2} & \leqslant C \int_{\rho-s}^{\rho} \int_{\partial B^{2}\left(b_{j}, \mu s\right)} \frac{1}{\varepsilon_{n}^{2}}\left(1-\left|\varphi_{n}^{\prime}\right|^{2}\right)^{2} \\
& \leqslant C \log \frac{1}{\varepsilon_{n}} .
\end{aligned}
$$

In $\frac{\rho-s}{\rho} \times B^{2}\left(b_{j}, \mu s\right)$ we have

$$
\begin{aligned}
\left|\nabla_{T} \varphi_{n}^{\prime}\right| & \leqslant C\left[\left|\frac{1}{l} \frac{\partial \varphi_{n}^{\prime}}{\partial \theta}\right|+\left|\frac{\partial \varphi_{n}^{\prime}}{\partial l}\right|\right] \\
& \leqslant C\left[\left|\frac{1}{\mu s} \frac{\partial \psi_{n}^{j}}{\partial \theta}\right|+\frac{1}{\mu s}\left|\psi_{n}^{j}\right|+\left|\frac{1}{\mu s} \frac{\partial\left|\varphi_{n}\right|}{\partial \theta}\right|(\theta, \mu s, \rho)\right. \\
& \left.+\frac{1}{\mu s}\left(\left|\varphi_{n}\right|(\theta, \mu s, \rho)-1\right)\right] .
\end{aligned}
$$

The integration of $(6.44)$ in $\frac{\rho-s}{\rho} B^{2}\left(b_{j}, \mu s\right)$ gives, in view of $(6.35)$

$$
\begin{aligned}
\int_{\frac{\rho-s}{\rho} B^{2}\left(b_{j}, \mu s\right)} & \left|\nabla+\varphi_{n}^{\prime}\right|^{2} \leqslant \\
& \leqslant C \int_{0}^{\frac{\rho-s}{\rho} \mu s} \int_{0}^{2 \pi}\left(\left|\nabla \varphi_{n}\right|^{2}(\theta, \mu s, \rho)+\frac{C}{s}\right) l d l d \theta
\end{aligned}
$$

and note that $|1-| \varphi_{n}^{\prime}|(\theta, l, \rho-s)| \leqslant|1-| \varphi_{n}|(\theta, \mu s, \rho)|$ thus we have, using (6. 36),

$$
\begin{aligned}
& \int_{\frac{\rho-s}{\rho} B^{2}(b j, \mu s)} \frac{1}{\varepsilon_{n}^{2}}\left(1-\left|\varphi_{n}^{\prime}\right|^{2}\right)^{2} \\
& \leqslant \int_{0}^{\frac{\rho-s}{\rho} \mu s} \int_{0}^{2 \pi} \frac{1}{\varepsilon_{n}^{2}}\left(1-\left|\varphi_{n}\right|^{2}\right)^{2}(\theta, \mu s, \rho) l d l d \theta \\
& \leqslant \int_{0}^{\frac{\rho-s}{\mu} s} \int_{0}^{2 \pi} \frac{l}{\mu s} d l \int_{\partial\left(B(b j, \mu s) \cap \partial B_{\rho}\left(x_{0}\right)\right)} \frac{1}{\varepsilon_{n}^{2}}\left(1-\left|\varphi_{n}\right|^{2}\right)^{2} \leqslant C \log \frac{1}{\varepsilon_{n}} \\
& \text { Esaim : Cocv June 1996, Vol.1, Pr. } 77-167
\end{aligned}
$$


Finally, let $C_{k}^{j}$ be the homotetic of $C^{j}$ by the homotetie of centre $b_{j}^{s}$ and rate $k$, we have

$$
\begin{aligned}
\int_{C^{j}}\left|\nabla \varphi_{n}^{\prime}\right|^{2} & \leqslant C \int_{v=0}^{s} d v \int_{\partial C_{v / s}^{j}}\left|\nabla_{\top} \varphi_{n}\left(\pi^{j}(x)\right)\right|^{2}\left|\nabla \pi^{j}\right|^{2}(x) \\
& \leqslant C \int_{v=0}^{s} \frac{s^{2}}{v^{2}} d v \int_{\partial C_{v / s}^{j}}\left|\nabla \top \varphi_{n}\left(\pi^{j}(x)\right)\right|^{2} \\
& \leqslant C s \int_{\partial C^{j}}\left|\nabla_{T} \varphi_{n}\right|^{2} \leqslant C s \log \frac{1}{\varepsilon_{n}}
\end{aligned}
$$

Moreover we have

$$
\begin{aligned}
\int_{C^{j}} \frac{1}{\varepsilon_{n}^{2}}\left(1-\left|\varphi_{n}^{\prime}\right|^{2}\right)^{2} & \leqslant C \int_{0}^{s} \frac{1}{\varepsilon_{n}^{2}} \int_{\partial C_{v / s}^{j}}\left(1-\left|\varphi_{n}\right|^{2}\right)^{2}\left(\pi^{j}(x)\right) \\
& \leqslant C \int_{0}^{s} \frac{v^{2}}{s^{2}} d v \int_{\partial C^{j}} \frac{1}{\varepsilon_{n}^{2}}\left(1-\left|\varphi_{n}\right|^{2}\right)^{2} \\
& \leqslant C s \int_{\partial C^{j}} \frac{1}{\varepsilon_{n}^{2}}\left(1-\left|\varphi_{n}\right|^{2}\right)^{2} \leqslant C s \log \frac{1}{\varepsilon_{n}}
\end{aligned}
$$

Combining $(6.40),(6.41),(6.47)$ and $(6.48)$ we obtain that $\varphi_{n}^{\prime}$ verifies

$$
\int_{B_{\rho}\left(x_{0}\right) \backslash B_{\rho-s}\left(x_{0}\right)}\left|\nabla \varphi_{n}^{\prime}\right|^{2}+\frac{1}{2 \varepsilon_{n}^{2}}\left(1-\left|\varphi_{n}^{\prime}\right|^{2}\right)^{2} \leqslant C s \log \frac{1}{\varepsilon_{n}} .
$$

Moreover $\left|\varphi_{n}^{\prime}\right| \geqslant \frac{1}{2}$ on $\partial B_{\rho-s}\left(x_{0}\right)$ and from $(6.45)$ we deduce

$$
\int_{\partial B_{\rho-s}\left(x_{0}\right)}\left|\nabla_{T} \varphi_{n}^{\prime}\right|^{2} \leqslant C(r, s),
$$

and from (6. 46)

$$
\int_{\partial B_{\rho-s}\left(x_{0}\right)} \frac{1}{\varepsilon_{n}^{2}}\left(1-\left|\varphi_{n}^{\prime}\right|^{2}\right)^{2} \leqslant C \log \frac{1}{\varepsilon_{n}} .
$$

Now we fill-up the ball $B_{\rho-s}\left(x_{0}\right)$ as we did at the end of the proof of Lemma 3.1.

Take the harmonic extension of $\psi_{n}$, where $\psi_{n}$ verifies $e^{i \psi_{n}}=\varphi_{n}^{\prime} /\left|\varphi_{n}^{\prime}\right|$ on $\partial B_{\rho-s}\left(x_{0}\right)$ and if $\xi_{n}$ is the solution of

$$
\left\{\begin{array}{l}
-\varepsilon_{n}^{2} \Delta \xi_{n}+\xi_{n}=1 \quad \text { in } B_{\rho-s}\left(x_{0}\right) \\
\xi_{n}=\left|\varphi_{n}^{\prime}\right| \quad \text { on } \partial B_{\rho-s}\left(x_{0}\right)
\end{array}\right.
$$

One verifies that $\varphi_{n}^{\prime}=\xi_{n} e^{i \psi_{n}}$ in $B_{p-s}\left(x_{0}\right)$ is such that

$$
\int_{B_{\rho-s}\left(x_{0}\right)}\left|\nabla \varphi_{n}^{\prime}\right|^{2}+\frac{1}{2 \varepsilon_{n}^{2}}\left(1-\left|\varphi_{n}^{\prime}\right|^{2}\right)^{2} \leqslant C(r, s) .
$$


Combining $(6.49)$ and $(6.52)$ we have extended $\varphi_{n}$ by $\varphi_{n}^{\prime}$ in such a way that

$$
\int_{B_{\rho}\left(x_{0}\right)}\left|\nabla \varphi_{n}^{\prime}\right|^{2}+\frac{1}{2 \varepsilon_{n}^{2}}\left(1-\left|\varphi_{n}^{\prime}\right|^{2}\right)^{2} \leqslant C s \log \frac{1}{\varepsilon_{n}}+C(r, s) .
$$

Since $h_{n}$ weakly converges in $W^{1, p}\left(B_{\rho}\left(x_{0}\right)\right)$ for any $p<\frac{3}{2}, h_{n}$ strongly converges in $L^{2}\left(B_{\rho}\left(x_{0}\right)\right)$ thus we have

$$
\frac{1}{\rho} \int_{B_{\rho}\left(x_{0}\right)}\left|\nabla_{\tilde{A}_{n}} \varphi_{n}^{\prime}\right|^{2}+\frac{1}{2 \varepsilon_{n}^{2}}\left(1-\left|\varphi_{n}^{\prime}\right|^{2}\right)^{2}+\left|h_{n}\right|^{2} \leqslant C \frac{s}{\rho} \log \frac{1}{\varepsilon_{n}}+C(r, s) .
$$

Choose now $s$ small enough such that $\frac{C s}{\rho}<\frac{\eta}{2}$ then we obtain that, for $\varepsilon_{n}$ sufficiently small,

$$
\frac{1}{\rho} \int_{B_{\rho}\left(x_{0}\right)}\left|\nabla_{\tilde{A}_{n}} \varphi_{n}\right|^{2}+\frac{1}{2 \varepsilon_{n}^{2}}\left(1-\left|\varphi_{n}\right|^{2}\right)^{2}+\left|h_{n}\right|^{2}<\eta \log \frac{\rho}{\varepsilon_{n}}
$$

This implies by Lemma 3.1 that $\left|\varphi_{n}\right| \geqslant \frac{1}{2}$ in $B_{r / 4}\left(x_{0}\right) \subset B_{\rho / 2}\left(x_{0}\right)$, and $h_{n}$ strongly converges in $H^{1}\left(B_{r / 4}\left(x_{0}\right)\right)$.

\section{The Limiting Singular SET IDENTIFIED AS A MinimaL CONNECTION}

In this part we conclude the proof of the theorem 1.2 stated in the introduction by proving the following theorem

Theorem 7.1. The 1-dimensional rectifiable current $I L$ given by the theorem 5.1 is a minimal connection between the $\left(x_{i}, d_{i}\right)_{i=1 \ldots n}$.

\section{Proof of theorem 7.1.}

We denote by $M$ the rectifiable set associated to $I L$, by $\xi(y)$ the approximate tangent vector (with the orientation given by $I L$ ) at $y \in M$ and by $\theta(y)$ the positive integer multiplicity of $I L$ at the same point. The rectifiability of $M$ implies that it can be included in a countable union of 1-dimensional disjoint submanifolds of $\mathbb{R}^{3}$ (i.e $M \subset \cup_{i=1}^{\infty} \mathcal{C}_{i}$ ) modulo an $\mathcal{H}^{1}$ - measure zero set that we do not consider in working with $I L$. We have, using the notation $M_{i}=M \cap \mathcal{C}_{i}$

$$
L=M(I L)=\int_{M} \theta d \mathcal{H}^{1}=\sum_{i=1}^{\infty} \int_{M \cap \mathcal{C}_{i}} \theta d \mathcal{H}^{1} .
$$

Let $\alpha>0$, we claim that for $n$ sufficiently large we have

$$
\int_{M_{\bar{x}}}\left|\nabla_{\tilde{A}_{n}} \varphi_{n}\right|^{2}+\frac{1}{2 \varepsilon_{n}^{2}}\left(1-\left|\varphi_{n}\right|^{2}\right)^{2}+\left|h_{n}\right|^{2} \geqslant 2 \pi(L-\alpha) \log \frac{1}{\varepsilon_{n}}-C(\alpha)(7.1)
$$

where $C(\alpha)$ does not depend on $n$.

Let $N \in \mathbb{N}$ be such that

$$
\sum_{i \geqslant N} \int_{M_{i}} \theta d \mathcal{H}^{1}<\alpha
$$

For $i \leqslant N$ and $z \in \mathcal{C}_{i}$ denote by $N_{i}(z)$ the plane passing by $z$ perpendicular to $\mathcal{C}_{i}$ and by $D_{i}(z, s)$ the two dimensional disk in this plane of centre $z$ and Esaim: CoCv June 1996, Vol.1, PP. 77-167 
radius s. $z$ also denotes sometimes the normal coordinate along $\mathcal{C}_{i}$.

Let $i \leqslant N$ be given. For $s>0$ sufficiently small the $D_{i}(z, s)$ for $z \in \mathcal{C}_{i}$ are disjoint and $\cup_{z \in \mathcal{C}_{i}} D_{i}(z, s)$ is a regular open tubular neighborhood of $\mathcal{C}_{i}$ denoted by $U_{i}(s)$. One verifies, using the coarea formula, that

$$
\begin{gathered}
\forall f \in L^{1}\left(U_{i}(s)\right) \\
\int_{U_{i}(s)} f=\int_{\mathcal{C}_{i}} d z \int_{D_{i}(z, s)} f(x)\left|1-C_{i}(z) \nu(z) \cdot(x-z)\right| d \mathcal{H}^{2}
\end{gathered}
$$

where $C_{i}(z)$ is the curvature of $\mathcal{C}_{i}$ at $z$ and $\nu(z)$ is first normal of $\mathcal{C}_{i}$ at $z$ when $C_{i}(z) \neq 0$.

Choose $s$ sufficiently small such that

$$
\sup _{i \leqslant N}\left[\sup _{z \in \mathcal{C}_{i}} C_{i}(z)\right] \times s \leqslant \alpha
$$

Let $\mathcal{A}_{i}^{N}(s)$ for $i \leqslant N$ be the following subset of $\mathcal{C}_{i}$

$$
\mathcal{A}_{i}^{N}(s)=\left\{z \in \mathcal{C}_{i} / \exists j \neq i, j \leqslant N \text { s.t. } D_{i}(z, s) \cap \cup_{j}(s) \neq \emptyset\right\}
$$

Since the $\mathcal{C}_{i}, i \leqslant N$ are disjoint submanifolds of $\mathbb{R}^{3}$, for $s$ chosen sufficiently small we can ensure

$$
\forall i \leqslant N \quad \int_{\mathcal{C}_{i} \cap \mathcal{A}_{i}^{N}(s)} \theta d \mathcal{H}^{1} \leqslant \frac{\alpha}{N}
$$

Denote by $\mathcal{B}_{i}^{N}(s)$, for $i \leqslant N$, the following subset of $\mathcal{C}_{i}$

$$
\mathcal{B}_{i}^{N}(s)=\left\{z \in \mathcal{C}_{i} \backslash \mathcal{A}_{i}^{N}(s) \quad / \exists j>N, \exists x \in M_{j} \cap D_{i}(z, s) \quad \text { s.t. } \quad \theta(x) \neq 0\right\}
$$

For $i \leqslant N$ and $j>N$ we consider the set

$$
R_{i}^{j}(s)=M_{j} \cap U_{i}(s) \backslash\left[U_{i}(s) \cap \underset{k \neq i k \leqslant N}{\cup} U_{k}(s)\right] .
$$

Clearly the $R_{i}^{j}(s)$ are all disjoint. Moreover we have

$$
\mathcal{H}^{1}\left(\mathcal{B}_{i}^{N}(s)\right) \leqslant \sum_{j>N} \int_{R_{i}^{j}(s)} \theta d \mathcal{H}^{1}
$$

Since $\sum_{i \leqslant N} \int_{\mathcal{C}_{i}} \theta d \mathcal{H}^{1}<+\infty$, there exists $\beta>0$ independent of $i \leqslant N$ such that

$$
\forall i \leqslant N \quad \mathcal{H}^{1}\left(\mathcal{B}_{i}^{N}(s)\right) \leqslant \beta \Rightarrow \int_{\mathcal{B}_{i}^{N}(s)} \theta d \mathcal{H}^{1} \leqslant \frac{\alpha}{N}
$$

We claim that, for $s$ sufficiently small, $\mathcal{H}^{1}\left(\mathcal{B}_{i}^{N}(s)\right) \leqslant \beta$ for $i \leqslant N$. Indeed, let $N^{\prime}$ such that

$$
\sum_{j>N^{\prime}} \int_{M_{j}} \theta d \mathcal{H}^{1} \leqslant \frac{\beta}{2 N}
$$

this implies clearly that

$$
\forall i \leqslant N \quad \sum_{j>N^{\prime}} \int_{R_{i}^{j}(s)} \theta d \mathcal{H}^{1} \leqslant \frac{\beta}{2 N} .
$$


Fix $N^{\prime}$ such that $(7.8)$ holds. Since the $\mathcal{C}_{j}, N<j \leqslant N^{\prime}$ are regular curves disjoint from the $\mathcal{C}_{i} \quad 1 \leqslant i \leqslant N$ for $s$ sufficiently small $\sum_{j=N+1}^{N^{\prime}} \mathcal{H}^{1}\left(R_{i}^{j}(s)\right)$ is as small as we want for any $i \leqslant N$ thus we can ensure

$$
\forall i \leqslant N \quad \sum_{j=N+1}^{N^{\prime}} \int_{R_{i}^{j}(s)} \theta d \mathcal{H}^{1} \leqslant \frac{\beta}{2 N} .
$$

Combining (7.9), (7. 10), (7.6) and (7. 7) for $s$ sufficiently small we have

$$
\forall i \leqslant N \quad \int_{\mathcal{B}_{i}^{N}(s)} \theta d \mathcal{H}^{1} \leqslant \frac{\alpha}{N} .
$$

Fix $s>0$ such that (7. 5) and (7. 11) hold. For $t>0$ to be chosen sufficiently small bellow, consider the lattice $t \mathbb{Z}^{3}$ in $\mathbb{R}^{3}$ except the points included in $\cup_{i=1}^{n} B\left(x_{i}, \frac{t}{\eta}\right)$, where $\eta$ is the constant given by Lemma 3.1. Because of Lemma 3.1 we can put the bad set out of $\cup_{i=1}^{n} B\left(x_{i}, \frac{t}{\eta}\right)\left(\right.$ i.e., $y \notin \cup_{i=1}^{n} B\left(x_{i}, \frac{t}{\eta}\right)$ such that $\left.\left|\varphi_{n}\right|(y) \leqslant \frac{1}{2}\right)$ in $C / t$ cubes $C\left(2 t, y_{l}\right)$ where $C$ does not depend neither on $n$ nor on $t$ and where the $y_{l}$ are points of the lattice.

Extract a subsequence such that the bad cubes $C\left(2 t, y_{l}\right)$ are always the same in $\cup_{i=1}^{N} U_{i}(s)$.

First of all for $t$ sufficiently small we can ensure

$$
\int_{M \cap \cup_{i=1}^{n} B\left(x_{i}, t / \eta\right)} \theta d \mathcal{H}^{1} \leqslant \alpha .
$$

Denote by $N_{t}^{i}(z)$, for $i \leqslant N$ and $z \in \mathcal{C}_{i}$, the cardinal of the following set

$$
N_{t}^{i}(z)=\#\left\{y_{l} / C\left(2 t, y_{l}\right) \cap D_{i}(z, s) \neq \emptyset\right\}
$$

Since a cube $C\left(2 t, y_{l}\right)$ can intersect $D_{i}(z, s)$ for $z$ in an interval of size at most $4 t$ we obtain that

$$
\int_{\mathcal{C}_{i}} N_{t}^{i}(z) d \mathcal{H}^{1} \leqslant C^{i}
$$

where $C^{i}$ does not depend on $t$. Consider $\beta$ such that

$$
\forall E \subset \mathcal{C}_{i} \quad \mathcal{H}^{1}(E) \leqslant \beta \Rightarrow \int_{\mathcal{C}_{i} \cap E} \theta d \mathcal{H}^{1} \leqslant \frac{\alpha}{N}
$$

Since $C^{i}$ does not depend on $t$, there exists $N^{i} \in \mathbb{N}$ independent on $t$ such that

$$
\forall i \leqslant N \quad \mathcal{H}^{1}\left(\left\{z \in \mathcal{C}_{i} \quad \text { s.t. } \quad N_{t}^{i}(z) \geqslant N^{i}\right\}\right) \leqslant \beta
$$

where $\beta$ is given by (7. 14) independently of $i \leqslant N$.

Let $\bar{N}=\max _{i \leqslant N} N^{i}$. Denote by $\mathcal{C}_{i}^{N}(s, t)$ the set

$$
\mathcal{C}_{i}^{N}(s, t)=\left\{z \in \mathcal{C}_{i} \text { s.t. } N_{t}^{i}(z) \geqslant \bar{N}\right\}
$$

For $t$ verifying

$$
0<t<\frac{s}{4 \bar{N}+4}
$$


we can ensure that there exists $k_{z} \in[1 ; 2 \bar{N}+1]$ for any $z \in \mathcal{C}_{i} \backslash \mathcal{C}_{i}^{N}(s, t)$ such that the annulus

$$
L_{i}(z, s)=D_{i}\left(z ; \frac{k_{z}+1}{2 \bar{N}+2} s\right) \backslash D_{i}\left(z ; \frac{k_{z}}{2 \bar{N}+2} s\right)
$$

does not intersect the bad set (i.e. $\cup_{l \leqslant \frac{c}{t}} C\left(2 t, y_{l}\right)$ ). Fix $t$ verifying (7. 12) and (7. 16). From part 6 we know that $\varphi_{n}$ tends strongly in $H^{1}$ to $\varphi_{\star}$ in the complement of the bad set $\cup_{l \leqslant \frac{c}{t}} C\left(2 t, y_{l}\right)$ in $\cup_{i \leqslant N} U_{i}(s)$. Thus for $n$ sufficiently large we have

$$
\begin{gathered}
\forall i \leqslant N \quad \forall z \in \mathcal{C}_{i} \backslash \mathcal{C}_{i}^{N}(s, t) \\
\operatorname{deg}\left(\frac{\varphi_{n}}{\left|\varphi_{n}\right|} ; \partial D_{i}\left(\frac{k z}{2 \bar{N}+2} s, z\right)=\operatorname{deg}\left(\varphi_{\star} ; \partial D_{i}\left(\frac{k_{z}}{2 \bar{N}+2} s, z\right)\right)\right.
\end{gathered}
$$

Moreover using Lemma A.5 we have

$$
\left\{\begin{array}{l}
\forall i \leqslant N, \quad \mathcal{H}^{1}-\text { a.e. } z \in \mathcal{C}_{i} \backslash \mathcal{A}_{i}^{N} \cup \mathcal{B}_{i}^{N} \cup \mathcal{C}_{i}^{N} \\
\operatorname{deg}\left(\varphi_{\star} ; \partial D_{i}\left(\frac{k_{z} s}{2 \bar{N}+2} s, z\right)\right)=-\theta(z)
\end{array}\right.
$$

Let $\alpha^{\prime}=1-\alpha$, using Lemma 6.1 the bad set in $\cup_{l \leqslant \frac{c}{t}} C\left(2 t, y_{l}\right)$ can be put in $C\left(\alpha^{\prime}\right) / \varepsilon_{n}^{\alpha^{\prime}}$ disjoints open sets of size $\approx \varepsilon_{n}^{\alpha^{\prime}}$ verifying conditions $1,2, \ldots, 6$. Denote by $P_{\alpha^{\prime}}^{i}(z)$ for $i \leqslant N$ and $z \in \mathcal{C}_{i}$ the cardinal of the following set

$$
P_{\alpha^{\prime}}^{i}(z)=\#\left\{R_{l} / R_{l} \cap D_{i}(z, s) \neq \emptyset\right\} \quad .
$$

Since each $R_{l}$ given by Lemma 6.1 for any $\alpha^{\prime}$ can intersect $D_{i}(z, s)$ for $z$ in an interval of size at most $4 \varepsilon^{\alpha^{\prime}}$ we obtain that

$$
\int_{\mathcal{C}_{i}} P_{\alpha^{\prime}}^{i}(z) d \mathcal{H}^{1} \leqslant C^{i}\left(\alpha^{\prime}\right)
$$

where $C^{i}\left(\alpha^{\prime}\right)$ does not depend on $\varepsilon_{n}$. Consider once again $\beta$ given by ( 7 . 14), there exists $P_{\alpha^{\prime}}^{i}$ independent of $\varepsilon_{n}$ such that

$$
\forall i \leqslant N \quad \mathcal{H}^{1}\left(\left\{z \in \mathcal{C}_{i} \text { s.t. } P_{\alpha^{\prime}}^{i}(z) \geqslant P_{\alpha^{\prime}}^{i}\right\}\right) \leqslant \beta
$$

Let $\bar{P}_{\alpha^{\prime}}=\max _{i \leqslant N} P_{\alpha^{\prime}}^{i}$ and denote by $\mathcal{D}_{i}^{N}(s)$ the set

$$
\mathcal{D}_{i}^{N}(s)=\left\{z \in \mathcal{C}_{i} \text { s.t. } P_{\alpha^{\prime}}^{i}(z) \geqslant \bar{P}_{\alpha^{\prime}}\right\} \text {. }
$$

Using Lemma A.6 for $\mathcal{H}^{1}$ a.e. $z \in \mathcal{C}_{i} \backslash \mathcal{A}_{i}^{N} \cup \mathcal{B}_{i}^{N} \cup \mathcal{C}_{i}^{N} \cup \mathcal{D}_{i}^{N}$ we have in any trivialisation of $E$ over $D_{i}(z, s)$

$$
\begin{aligned}
\int_{D_{i}\left(\frac{k_{z}+1}{2 N+2} s, z\right) \backslash \cup R_{l}}\left|\nabla \frac{\varphi_{n}}{\left|\varphi_{n}\right|}\right|^{2} & \geqslant 2 \pi \theta(z) \alpha^{\prime} \log \frac{1}{\varepsilon_{n}}- \\
& -2 \pi \log \left(\frac{s}{2 \bar{N}+2}\right)-2 \pi\left(\bar{P}_{\alpha^{\prime}}\right)^{5}
\end{aligned}
$$

where $\mathrm{d}$ is the maximal degree of $\varphi_{n} /\left|\varphi_{n}\right|$ around each $R_{l}$ and is uniformly bounded independently of $\varepsilon_{n}$ because of conditions 4 and 5 of Lemma 4.1. 
By lowering $\eta$ in Lemma 3.1 we can impose that $\left|\varphi_{n}\right| \geqslant \alpha^{\prime}$ in the complement of the $R_{l}$. Thus we have

$$
\begin{aligned}
& \mathcal{H}^{1}-\text { a.e. } z \in \mathcal{C}_{i} \backslash \mathcal{A}_{i}^{N} \cup \mathcal{B}_{i}^{N} \cup \mathcal{C}_{i}^{N} \cup \mathcal{D}_{i}^{N} \\
& \int_{D_{i}(z, s)}\left|\nabla \varphi_{n}\right|^{2} \geqslant 2 \pi \theta(z) \alpha^{\prime 3} \log \frac{1}{\varepsilon_{n}}-2 \pi \alpha^{\prime 2} \log \left(\frac{s}{2 \bar{N}+2}\right)-2 \pi\left(\bar{P}_{\alpha^{\prime}}\right)^{5^{5}}
\end{aligned}
$$

Since we are working in $\cup_{i=1}^{N} U_{i}(s) \backslash U_{i=1}^{n} B\left(x_{i}, \frac{t}{\eta}\right)$ we have the strong convergence $\tilde{A}_{n} \rightarrow \tilde{A}$ in $L^{2}$ thus

$$
\int_{\cup_{i=1}^{N} U_{i}(s) \backslash \cup_{i=1}^{n} B\left(x_{i}, t / \eta\right)}\left|\tilde{A}_{n}\right|^{2} \leqslant C
$$

where $C$ does not depend on $\varepsilon_{n}$. Integrating (7. 21) along the $\mathcal{C}_{i} \backslash \mathcal{A}_{i}^{N} \cup \mathcal{B}_{i}^{N} \cup$ $\mathcal{C}_{i}^{N} \cup \mathcal{D}_{i}^{N}$ using (7.3) and (7. 22), since we have the bound using (7. 5), (7. 11), (7. 15) and (7. 20)

$$
\int_{\mathcal{A}_{i}^{N} \cup \mathcal{B}_{i}^{N} \cup \mathcal{C}_{i}^{N} \cup \mathcal{D}_{i}^{N}} \theta d \mathcal{H}^{1} \leqslant \frac{4 \alpha}{N}
$$

we get, using also (7. 12),

$$
\begin{aligned}
& \int_{\cup_{i=1}^{N} \cup(s) \backslash \cup_{i=1}^{n} B\left(x_{i}, t / \eta\right)}\left|\nabla_{\tilde{A}} \varphi\right|^{2} \geqslant \\
& 2 \pi \alpha^{\prime 3}\left(\int_{\cup_{i=1}^{N} M_{i}} \theta d \mathcal{H}^{1}-5 \alpha\right) \log \frac{1}{\varepsilon_{n}}-C(\alpha)
\end{aligned}
$$

where $C(\alpha)$ does not depend on $N$. Since $\alpha^{\prime}=1-\alpha$ considering (7. 2), (7. 23) implies (7. 1).

Combining the upper bound of the total energy given by Lemma 2.1 and ( 5 . $47)$ which says that $I L$ is a connection between the $\left(x_{i}, d_{i}\right)_{i=1 \ldots n}$, the lower bound (7. 1) implies that $I L$ is a minimal connection.

\section{APPENDIX A. APPENDIX}

Lemma A.1. Let $\varphi, \eta, \xi$ be 0,1 and 2 -forms of $B_{1} \subset \mathbb{R}^{3}$ we have

$$
\begin{gathered}
\frac{\partial}{\partial r} d \varphi=d \frac{\partial \varphi}{\partial r}-\frac{d \varphi}{r}+\frac{\partial \varphi}{\partial r} d(\log r) \\
\frac{\partial}{\partial r} d \eta=d \frac{\partial \eta}{\partial r}-\frac{d \eta}{r}+d(\log r) \wedge \frac{\partial \eta}{\partial r}
\end{gathered}
$$

and

$$
\frac{\partial}{\partial r} d \xi=d \frac{\partial \xi}{\partial r}-\frac{d \xi}{r}+d(\log r) \wedge \frac{\partial \xi}{\partial r}
$$


Proof of Lemma A.1.

$$
\begin{aligned}
\frac{\partial}{\partial r} d \varphi & =\sum_{i} \frac{\partial}{\partial r} \frac{\partial \varphi}{\partial x_{i}} d x_{i}=\sum_{i k} \frac{\partial}{\partial x_{k}} \frac{\partial \varphi}{\partial x_{i}} \frac{x_{k}}{|x|} d x_{i} \\
& =\sum_{i k} \frac{\partial}{\partial x_{i}} \frac{\partial \varphi}{\partial x_{k}} \frac{x_{k}}{|x|} d x_{i} \\
& =\sum_{i} \frac{\partial}{\partial x_{i}} \frac{\partial \varphi}{\partial r} d x_{i}-\sum_{i k} \frac{\partial \varphi}{\partial x_{k}} \frac{\partial}{\partial x_{i}}\left(\frac{x_{k}}{|x|}\right) d x_{i} \\
& =d\left(\frac{\partial \varphi}{\partial r}\right)-\frac{1}{r} d \varphi+\frac{\partial \varphi}{\partial r} d(\log r) .
\end{aligned}
$$

The two other equalities are proved in the same way by direct computations

Lemma A.2. Let $\varphi$ and $\eta$ be 0 and 1 forms in $B_{1} \subset \mathbb{R}^{3}$. for $r>0$ let $d_{\top}$ and $(*)$ the exterior derivative and the Hodge operator on $\partial B_{r} \subset \mathbb{R}^{3}$ and let $\Delta_{r}$ be the Laplace-Beltrani operator on $\partial B_{r}$. We have

$$
\begin{aligned}
& d_{\top} \frac{\partial \varphi}{\partial r}=\frac{\partial}{\partial r} d_{\top} \varphi+\frac{1}{r} d_{\top \varphi} \\
& d_{\top} \frac{\partial \varphi}{\partial r}=\frac{\partial}{\partial r} d_{\top \eta} \eta \frac{1}{r} d_{\top} \eta
\end{aligned}
$$

Let $i_{r}$ the isometric embeding in $i_{r}: \partial B_{r} \rightarrow \mathbb{R}^{3}$

$$
\frac{\partial}{\partial r}(*) i_{r}^{\#} \eta=(*) i_{r}^{\#} \frac{\partial \eta}{\partial r}
$$

and

$$
\frac{\partial}{\partial r} \Delta_{r} \varphi-\Delta_{r} \frac{\partial \varphi}{\partial r}=-\frac{2}{r} \Delta_{r} \varphi
$$

\section{Proof of Lemma A.2.}

By definition

thus by Lemma A.1

$$
d_{\top} \frac{\partial \varphi}{\partial r}=d \frac{\partial \varphi}{\partial r}-\frac{\partial}{\partial r} \frac{\partial \varphi}{\partial r} d r
$$

$$
\begin{aligned}
d_{\top} \frac{\partial \varphi}{\partial r} & =\frac{\partial}{\partial r} d \varphi+\frac{d \varphi}{r}-\frac{\partial \varphi}{\partial r} \frac{d r}{r}-\frac{\partial^{2} \varphi}{\partial r^{2}} d r \\
& =\frac{\partial}{\partial r}\left(d_{\top \varphi}+\frac{\partial \varphi}{\partial r} d r\right)+\frac{d \varphi}{r}-\frac{\partial \varphi}{\partial r} \frac{d r}{r}-\frac{\partial^{2} \varphi}{\partial r^{2}} d r \\
& =\frac{\partial}{\partial r}\left(d_{\top} \varphi\right)+\frac{d \varphi}{r}-\frac{\partial \varphi}{\partial r} \frac{d r}{r} \\
& =\frac{\partial}{\partial r} d_{\top \varphi}+\frac{1}{r} d_{\top \varphi}
\end{aligned}
$$


Let

$$
\omega=* d r=\frac{x d y \wedge d z+y d z \wedge d x+z d x \wedge d y}{r}
$$

By definition

$$
d_{\top} \frac{\partial \eta}{\partial r}=\left\langle d \frac{\partial \eta}{\partial r}, \omega\right\rangle \omega
$$

thus

$$
\begin{aligned}
d_{\top} \frac{\partial \eta}{\partial r} & =\left\langle\frac{\partial}{\partial r} d \eta+\frac{d \eta}{r}-\frac{d r}{r} \wedge \frac{\partial \eta}{\partial r}, \omega\right\rangle \omega \\
& =\left\langle\frac{\partial}{\partial r} d \eta+\frac{d \eta}{r}, \omega\right\rangle \omega
\end{aligned}
$$

Since $\frac{\partial \omega}{\partial r}=0$ we have

$$
\begin{aligned}
d_{\top} \frac{\partial \eta}{\partial r} & =\frac{\partial}{\partial r}[\langle d \eta, \omega\rangle \omega]+\frac{1}{r}\langle d \eta, \omega\rangle \omega \\
& =\frac{\partial}{\partial r}\left(d_{\top} \eta\right)+\frac{1}{r} d_{\top} \eta .
\end{aligned}
$$

We have $(*) i_{r}^{\#} \eta=(*)(\eta \wedge d r)$, thus

$$
\begin{aligned}
\frac{\partial}{\partial r}(*) i_{r}^{\#} \eta=* \frac{\partial}{\partial r}(\eta \wedge d r) & =*\left(\frac{\partial \eta}{\partial r} \wedge d r\right) \\
& =(*) i_{r}^{\#} \frac{\partial \eta}{\partial r}
\end{aligned}
$$

Finally

$$
\begin{aligned}
\Delta_{r} \frac{\partial \varphi}{\partial r} & =(*) d_{\top}(*) d_{\top} \frac{\partial \varphi}{\partial r}=(*) d_{\top}(*)\left[\frac{\partial}{\partial r} d_{\top \varphi}+\frac{1}{r} d_{\top} \varphi\right] \\
& =(*) d_{\top} \frac{\partial}{\partial r}\left[(*) d_{\top} \varphi\right]+\frac{1}{r} \Delta_{r} \varphi \\
& =(*) \frac{\partial}{\partial r}\left[d_{\top}(*) d_{\top} \varphi\right]+\frac{1}{r}(*) d_{\top}(*) d_{\top} \varphi+\frac{1}{r} \Delta_{r} \varphi \\
& =\frac{\partial}{\partial r} \Delta_{r} \varphi+\frac{2}{r} \Delta_{r} \varphi
\end{aligned}
$$

Lemma A.3. Let $\varphi$ be a 0 -form on $B_{1} \subset \mathbb{R}^{3}$ taking its values into $\mathbb{C}$, we have

$$
\Delta_{r}\left(i \varphi, \frac{\partial \varphi}{\partial r}\right)-\frac{\partial}{\partial r}\left(i \varphi, \Delta_{r} \varphi\right)=2(*) d_{\top}\left[\left(\frac{\partial \varphi}{\partial r}, i(*) d_{\top \varphi}\right)\right]+\frac{2}{r}\left(i \varphi, \Delta_{r} \varphi\right)
$$




\section{Proof of Lemma A.3.}

$$
\begin{aligned}
\Delta_{r}\left(i \varphi, \frac{\partial \varphi}{\partial r}\right) & =(*) d_{\top}(*) d_{\top}\left(i \varphi, \frac{\partial \varphi}{\partial r}\right) \\
& =(*) d_{\top}(*)\left[\left(\frac{\partial \varphi}{\partial r}, i d_{\top} \varphi\right)+\left(i \varphi, d_{\top} \frac{\partial \varphi}{\partial r}\right)\right] \\
& =(*)\left(i d_{\top}(*) d_{\top} \varphi, \frac{\partial \varphi}{\partial r}\right)+(*)\left(d_{\top} \frac{\partial \varphi}{\partial r}, i(*) d_{\top}\right) \\
& +(*)\left(i d_{\top} \varphi,(*) d_{\top} \frac{\partial \varphi}{\partial r}\right)+(*)\left(i \varphi, d_{\top}(*) d_{\top} \frac{\partial \varphi}{\partial r}\right)
\end{aligned}
$$

where we have used the following notation, if $a$ and $b$ are two $\mathbb{C} 1$-form,

$$
(a, b)=\frac{1}{2}[a \wedge \bar{b}+\bar{a} \wedge b]
$$

and since $((*) a) \wedge b=((*) b) \wedge a$ we have

$$
((*) a, b)=((*) b, a) .
$$

This implies

$$
\begin{aligned}
\Delta_{r}\left(i \varphi, \frac{\partial \varphi}{\partial r}\right) & =\left(i \Delta_{r} \varphi, \frac{\partial \varphi}{\partial r}\right)+2(*)\left(d_{\top} \frac{\partial \varphi}{\partial r}, i(*) d_{\top} \varphi\right) \\
& +\left(i \varphi, \Delta_{r} \frac{\partial \varphi}{\partial r}\right),
\end{aligned}
$$

and finally

$$
\begin{aligned}
\Delta_{r}\left(i \varphi, \frac{\partial \varphi}{\partial r}\right) & =\left(i \Delta_{r} \varphi, \frac{\partial \varphi}{\partial r}\right)+2(*) d_{\top}\left[\left(\frac{\partial \varphi}{\partial r}, i(*) d_{\top} \varphi\right)\right] \\
& -2\left(\frac{\partial \varphi}{\partial r} i \Delta_{r} \varphi\right)+\left(i \varphi, \frac{\partial}{\partial r} \Delta_{r} \varphi\right)+\left(i \varphi, \frac{2}{r} \Delta_{r} \varphi\right) \\
& =\frac{\partial}{\partial r}\left(i \varphi, \Delta_{r} \varphi\right)-\left(i \frac{\partial \varphi}{\partial r}, \Delta_{r} \varphi\right)+\left(i \Delta_{r} \varphi, \frac{\partial \varphi}{\partial r}\right) \\
& -2\left(\frac{\partial \varphi}{\partial r}, i \Delta_{r} \varphi\right)+2(*) d_{\top}\left[\left(\frac{\partial \varphi}{\partial r}, i(*) d_{\top} \varphi\right)\right] \\
& +\frac{2}{r}\left(i \varphi, \Delta_{r} \varphi\right) .
\end{aligned}
$$

This yields the desired result.

Lemma A.4. Let $f$ and $\eta$ be 0 and 1 -forms in $B_{1} \subset \mathbb{R}^{3}$ and $\omega=* d r$, we have

and

$$
<\Delta(f \omega) ; \omega>=\Delta f-\frac{2}{r^{2}} f
$$

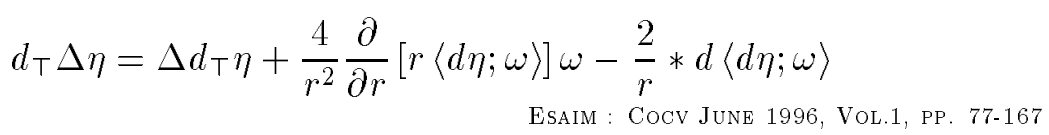




\section{Proof of Lemma A.4.}

We have

$$
\Delta(f \omega)=\Delta f \omega+2 \sum_{k} \frac{\partial f}{\partial k} \frac{\partial \omega}{\partial k}+f \Delta \omega .
$$

Moreover one verifies that $\Delta \omega=-\frac{2}{r^{2}} \omega$ and in the other hand $\forall k<\frac{\partial \omega}{\partial k} ; \omega>=0$ thus

$$
<\Delta(f \omega) ; \omega>=\Delta f-\frac{2}{r^{2}} f .
$$

By definition

$$
d_{\top} \Delta \eta=<d \Delta \eta ; \omega>\omega=<\Delta d \eta, \omega>\omega .
$$

In the other hand

$$
\begin{aligned}
\Delta d_{\top} \eta & =\Delta[<d \eta ; \omega>\omega]=\sum_{i} \Delta\left[<d \eta ; \omega>\frac{x_{i}}{r}\right] d x_{i+1} \wedge d x_{i-1} \\
& =2 \sum_{i} \nabla<d \eta ; \omega>\nabla\left(\frac{x_{i}}{r}\right) d x_{i+1} \wedge d x_{i-1} \\
& +\Delta<d \eta ; \omega>\omega-\frac{2}{r^{2}}\langle d \eta ; \omega>\omega .
\end{aligned}
$$

We have also

$$
\begin{aligned}
\Delta\langle d \eta ; \omega\rangle & =\langle d \Delta \eta ; \omega\rangle+2 \sum_{i} \nabla(d \eta)_{i} \nabla\left(\frac{x_{i}}{r}\right)+\langle d \eta ; \Delta \omega\rangle \\
& =\langle d \Delta \eta ; \omega\rangle+2 \sum_{i l} \frac{\partial}{\partial l}(d \eta)_{i} \frac{\partial}{\partial l}\left(\frac{x_{i}}{r}\right)-\frac{2}{r^{2}}\langle d \eta ; \omega\rangle \\
& =\langle d \Delta \eta ; \omega\rangle+2 \sum_{i} \frac{\partial}{\partial i}(d \eta)_{i} \frac{1}{r}+2 \sum_{i l} \frac{\partial}{\partial l}(d \eta)_{i} x_{i} \frac{\partial}{\partial l}\left(\frac{1}{r}\right) \\
& -\frac{2}{r^{2}}\langle d \eta ; \omega\rangle
\end{aligned}
$$

Clearly $\sum_{i} \frac{\partial(d \eta)_{i}}{\partial i}=* d(d \eta)=0$, and since $\frac{\partial}{\partial r}\left(\frac{x_{i}}{r}\right)=0$

$$
\begin{aligned}
-2 \sum_{i l} \frac{\partial}{\partial l}(d \eta)_{i} x_{i} \frac{x_{l}}{r^{3}} & =-\frac{2}{r} \sum_{l} \frac{x_{l}}{r} \frac{\partial}{\partial l}\left(\sum_{i}(d \eta)_{i} \frac{x_{i}}{r}\right) \\
& =-\frac{2}{r} \frac{\partial}{\partial r}\langle d \eta ; \omega\rangle .
\end{aligned}
$$

This implies

$$
\begin{aligned}
\Delta\langle d \eta ; \omega\rangle & =\langle d \Delta \eta ; \omega\rangle-\frac{2}{r} \frac{\partial}{\partial r}\langle d \eta ; \omega\rangle-\frac{2}{r^{2}}\langle d \eta ; \omega\rangle \\
& =\langle d \Delta \eta ; \omega\rangle-\frac{2}{r^{2}} \frac{\partial}{\partial r}[r\langle d \eta ; \omega\rangle] .
\end{aligned}
$$


Finally

$$
\begin{aligned}
\nabla<d \eta ; \omega\rangle . \nabla\left(\frac{x_{i}}{r}\right) & =\frac{1}{r} \frac{\partial}{\partial x_{i}}\langle d \eta ; \omega\rangle \\
& -\frac{x_{i}}{r^{2}} \frac{\partial}{\partial r}\langle d \eta ; \omega\rangle .
\end{aligned}
$$

Combining (A. 1), (A. 3) and (A. 4) we have

$$
\begin{aligned}
\Delta d_{\top \eta}= & \langle d \Delta \eta ; \omega\rangle \omega-\frac{2}{r^{2}} \frac{\partial}{\partial r}[r\langle d \eta ; \omega\rangle] \omega \\
& +\frac{2}{r} * d\langle d \eta ; \omega\rangle-\frac{2}{r} \frac{\partial}{\partial r}[\langle d \eta ; \omega\rangle] \omega \\
& -\frac{2}{r^{2}}\langle d \eta ; \omega\rangle \omega .
\end{aligned}
$$

This yields the desired result.

Lemma A.5. Let $\Omega$ and $\omega$ we be two bounded regular subsets of $\mathbb{R}^{2}$ such that $\Omega$ is simply connected and $\omega \subset \subset \Omega$. Let $a>0$ and denote by $C$ and $\mathcal{C}$ the following cylinders of $\mathbb{R}^{3}$.

$$
C=\omega \times(-a, a) \quad \text { and } \quad \mathcal{C}=\Omega \times(-a, a) .
$$

Let $T(\theta, M)$ be a 1-dimensionnal, finite mass, integer multiplicity rectifiable current with support included in $C$ and let $\psi$ be $W^{1,1}\left(\mathcal{C}, S^{1}\right)$ map such that $\psi$ is regular in $\mathcal{C} \backslash C$ and

$$
* d(i \psi, d \psi)=2 \pi \pi^{T} \quad \text { in } \quad \mathcal{D}_{1}(\mathcal{C})
$$

Let $\Gamma$ be a closed regular curve in $\Omega \backslash \omega$ and $\Sigma$ in $\Omega$ such that $\partial \Gamma=\Sigma$. Denote by $\Gamma_{t}, \Sigma_{t}, \omega_{t}$ and $\Omega_{t}$ the sets $\Gamma \times\{t\}, \Sigma \times\{t\}, \omega \times\{t\}$ and $\Omega \times\{t\}$ for $t \in(-a, a)$ then we have

$$
\text { for a.e. } t \in(-a, a) \quad \begin{aligned}
\operatorname{deg}_{\Gamma_{0}} \psi & =\operatorname{deg}_{\Gamma_{t}} \psi \\
& =-\sum_{y \in M \cap \Sigma_{t}} \operatorname{sgn}<\xi ; e_{3}>(y) \theta(y)
\end{aligned}
$$

where $e_{3}=(0,0,1)$ and $\Gamma_{t}$ is oriented by $\epsilon_{3}$.

\section{Proof of Lemma A.5.}

First of all we claim that

$$
\begin{gathered}
\text { for a.e } t \in(-a, a) \quad \sum_{y \in M \cap \omega_{t}} \theta(y)<+\infty \\
\text { and } \quad \forall y \in M \cap \omega_{t} \quad<\xi(y) ; e_{3}>\neq 0
\end{gathered}
$$

This can be proved using the coarea formula of Federer (see for instance [9] pages 15 and 34 ). Let $t$ be the function on $M$ which associates the third coordinate. This is a Lipshitz function in $M$ (which is rectifiable subset of 
$\mathbb{R}^{3}$ ) moreover for $\mathcal{H}^{1}$ a.e.y $\in M$ ap $J_{1} t(y)=\mid\left\langle\xi(y) ; e_{z}>\right|$ thus for any $\mathcal{H}^{1} L M$ integrable function $g$ on $M$ we have

$$
\begin{aligned}
\int_{M}\left|<\xi(y), e_{3}>\right| g d \mathcal{H}^{1} & =\int_{-a}^{a} \int_{M \cap \omega_{t}} g d \mathcal{H}^{0} d t \\
& =\int_{-a}^{a} \sum_{y \in M \cap \omega_{t}} g(y) d t
\end{aligned}
$$

Take first $g=\theta$, since $M(T)<+\infty$ we have

$$
\int_{-a}^{a} \sum_{y \in M \cap \omega_{t}} \theta(y) d t \leqslant M(\mathbb{T})
$$

For any $\alpha>0$ take $g_{\alpha}=\frac{\theta(y)}{\alpha+\mid<\dot{\xi}(y), e_{3}>}$, we have

$$
\int_{-a}^{+a} \sum_{y \in M \cap \omega_{t}} \frac{\theta(y)}{\alpha+\left|<\xi(y), e_{3}>\right|} \leqslant M(\mathbb{T}) .
$$

Thus, let

$$
E=\left\{\begin{array}{lc}
t \in(-a, a) \text { s.t. } & \sum_{y \in M \cap \omega_{t}} \theta(y)<+\infty \text { and } \\
\exists y \in M \cap \omega_{t} & \text { s.t. } \quad \theta(y) \neq 0 \text { and }\left\langle\xi(y), e_{3}>=0\right.
\end{array}\right\}
$$

from (A. 10) we deduce that

$$
\forall \alpha>0 \quad|E| \times \frac{1}{\alpha} \leqslant M(\mathbb{T})
$$

This implies (A. 7).

Since $\Gamma_{t}$ is homotopic to $\Gamma_{0}$ in $\mathcal{C} \backslash C$ we have clearly

$$
\operatorname{deg}_{\Gamma_{t}} \psi=\operatorname{deg}_{\Gamma_{0}} \psi \text {. }
$$

Let $t_{0} \in(-a, a)$ such that (A. 7) holds for $t=t_{0}$ and let $U$ be a tubular neighborhood of $\Gamma$ chosen sufficiently small such that

$$
U \subset \mathcal{C} \backslash C
$$

and $U$ is diffeomorphic to a neighborhood of the zero section of the normal bundle $N \Gamma$ of $\Gamma \subset \mathcal{C}$.

We can suppose that this diffeomorphism sends linearly each intersection of $U$ and any normal plane in $\mathcal{C}$ to $\Gamma$ (at $\gamma$ ) to a neigborhood of 0 in the corresponding fiber $\left(\pi^{-1} \gamma=N_{\gamma} \Gamma\right)$ of $N \Gamma$.

Let $\chi$ be the following function on $\Omega \times\left\{t_{0}\right\}$

$$
\left\{\begin{array}{l}
0 \leqslant \chi \leqslant 1 \\
\chi \equiv 0 \text { in } \Omega \times\left\{t_{0}\right\} \backslash \Sigma \times\left\{t_{0}\right\} \\
\chi \equiv 1 \text { in } \omega \times\left\{t_{0}\right\} \\
\operatorname{supp}(d \chi) \subset U \cap \Omega \times\left\{t_{0}\right\},
\end{array}\right.
$$


and define on all of $\mathcal{C} \chi(x, y, t) \equiv \chi\left(x, y, t_{0}\right)$.

Let $H$ be a non negative function of $C_{c}^{\infty}\left(\mathbb{R}_{\mathbb{R}_{+}}\right)$such that

$$
\left\{\begin{array}{l}
H \equiv 1 \text { in a neigborhood of } 0 \\
\int_{\mathbb{R}} H=1
\end{array}\right.
$$

Let $H_{\lambda}^{t_{0}}=\frac{1}{\lambda} H\left(\frac{t-t_{0}}{\lambda}\right)$ for $\lambda>0$ chosen sufficiently small

$$
\operatorname{supp}\left(H_{\lambda}^{t_{0}} \times d \chi \wedge d t\right) \subset U
$$

Let

$$
\eta_{\Gamma_{t_{0}}}=H_{\lambda}^{t_{0}} d \chi \wedge d t
$$

We clearly have $d \eta_{\Gamma_{t_{0}}}=0$, moreover let $\gamma \in \Gamma_{t_{0}}$ and let $P$ be the vertical plane (containing $e_{3}$ ) passing by $\gamma$, perpendicular to $\Gamma_{t_{0}}$ and oriented by the unit tangent vector $\dot{\gamma}$ of $\Gamma_{t_{0}}$ (oriented by $e_{3}$ i.e. such that $\dot{\gamma} \wedge e_{3}$ is the unit outward normal of $\left.\partial \Sigma_{t_{0}}\right)$ we have

$$
\begin{aligned}
\int_{P_{\gamma}} \eta_{\Gamma_{t_{0}}}=\int_{P_{\gamma}} d \chi \wedge H_{\lambda}^{t_{0}} d t & =\int_{P_{\gamma} \cap \Gamma_{t_{o}}} d \chi \times \int_{-a}^{a} H_{\lambda}^{t_{0}} d t \\
& =1
\end{aligned}
$$

where $P_{\gamma}$ is the component of $P \cap U$ which contains $\gamma$. Thus $\eta_{\Gamma_{t_{0}}}$ is the Thom Class of $N \Gamma_{t_{0}}$ (see [5] chapter I.6) and from proposition 6.24 of [5] this is the Poincaré dual of $\Gamma_{t_{0}}$ in $\mathcal{C}$. In the other hand, since $\operatorname{supp}\left(\eta_{\Gamma_{t_{0}}}\right) \subset U$, we have

$$
\int_{\mathcal{C}}(i \psi, d \psi) \wedge \eta_{\Gamma_{t_{0}}}=\int_{U}(i \psi, d \psi) \wedge \eta_{\Gamma_{t_{0}}}
$$

and since $(i \psi, d \psi)$ is regular in $U$ and $d(i \psi, d \psi)=0$ in $U$, the Poincaré duality implies

$$
\int_{\mathcal{C}}(i \psi, d \psi) \wedge \eta_{\Gamma_{t_{0}}}=\int_{\Gamma_{t_{0}}}(i \psi, d \psi)=2 \pi d e g_{\Gamma_{t_{0}}} \psi
$$

Remark that $\eta_{\Gamma_{t_{0}}}=d\left(\chi H_{\lambda}^{t_{0}} d t\right)$ thus, since $\operatorname{supp}\left(\chi H_{\lambda}^{t_{0}} d t\right) \subset \mathcal{C}$, applying (A. 5) we have

$$
\begin{aligned}
\int_{\mathcal{C}}(i \psi, d \psi) \wedge \eta_{\Gamma_{t_{0}}} & =-<* d(i \psi, d \psi) ; \chi H_{\lambda}^{t_{0}} d t> \\
& =-2 \pi<T ; \chi H_{\lambda}^{t_{0}} d t>
\end{aligned}
$$

By hypothesis supp $\mathbb{T} \subset C$ and $\chi \equiv 1$ in $C$ thus

$$
<\mathbb{T} ; \chi H_{\lambda}^{t_{0}} d t>=\int_{M} H_{\lambda}^{t_{0}}<\xi ; e_{z}>\theta d \mathcal{H}^{1}
$$


Applying the coarea formula (A.8) for $g=H_{\lambda}^{t_{0}} \frac{\left\langle\xi, e_{3}\right\rangle}{\left|\left\langle\xi, e_{3}\right\rangle\right|} \theta$ we have

$$
\begin{aligned}
<\mathbb{T} ; \chi H_{\lambda}^{t_{0}} d t> & =\int_{-a}^{+a} \sum_{y \in M \cap \omega_{t}} H_{\lambda}^{t_{0}}(t) \theta(y) \frac{\left\langle\xi, e_{3}\right\rangle}{\left|\left\langle\xi, e_{3}\right\rangle\right|} d t \\
& =\int_{-a}^{+a} \frac{1}{\lambda} H\left(\frac{t-t_{0}}{\lambda}\right) f(t) d t
\end{aligned}
$$

where $f$ is the $L^{1}$ function on $(-a, a)$ such that

$$
f(t)=\sum_{y \in M \cap \omega_{t}} \theta(y) \frac{\left.<\xi ; e_{3}\right\rangle}{\left|<\xi ; e_{3}\right\rangle \mid}
$$

For almost every to $t \in(-a, a)$

$$
\frac{1}{\lambda} \int_{t_{0}-\lambda}^{t_{0}+\lambda}\left|f(t)-f\left(t_{0}\right)\right| d t \rightarrow 0 \text { as } \quad \lambda \rightarrow+\infty
$$

Suppose the $t_{0}$ we have chosen verifying (A. 7) is also a Lebesgue point for $f$ verifying (A. 21), thus we have

$$
\begin{aligned}
\left|<T, \chi H_{\lambda}^{t_{0}} d t>-f\left(t_{0}\right)\right| & =\left|\int_{-a}^{+a} \frac{1}{\lambda} H\left(\frac{t-t_{0}}{\lambda}\right)\left(f(t)-f\left(t_{0}\right)\right) d t\right| \\
& \leqslant \frac{1}{\lambda}\|H\|_{L^{\infty}} \int_{t_{0}-\lambda}^{t_{0}+\lambda}\left|f(t)-f\left(t_{0}\right)\right| d t
\end{aligned}
$$

where we have used the fact that $\frac{1}{\lambda} \int_{-a}^{+a} H\left(\frac{t-t_{0}}{\lambda}\right)=1$. Combining (A. 16), (A. 17), (A. 19), (A. 20) (A. 21) and (A. 22) we get the desired result.

Lemma A.6. Let $0<R_{1}<R_{2}, 0<\eta<R_{1}, N$ points $a_{1}, \ldots, a_{N}$ in the ball $B_{R_{1}}(0)$ of $\mathbb{R}^{2}$ such that

$$
\begin{aligned}
& \forall 1 \leqslant i \leqslant N \quad B_{\eta}\left(a_{i}\right) \subset B_{R_{1}}(0) \\
& \forall i \neq j \quad B_{\eta}\left(a_{i}\right) \cap B_{\eta}(a j)=\emptyset
\end{aligned}
$$

and let $d_{1}, \ldots, d_{N}$ be $N$ integers in $\{-D,-D+1, \ldots, D-1, D\}$. For any $u: \bar{B}_{R_{2}}(0) \backslash \cup_{i=1}^{N} B_{\eta}\left(a_{i}\right)=\Omega_{\eta} \rightarrow S^{1}$ such that

$$
\operatorname{deg}_{\partial B_{\eta}\left(a_{i}\right)} u=d_{i}
$$

we have

$$
\int_{\Omega_{\eta}}|\nabla u|^{2} \geqslant 2 \pi\left|\sum_{i=1}^{N} d_{i}\right| \log \frac{R_{2}-R_{1}}{\eta}-2 \pi \log 2 D N^{5}
$$


Remark A.1. The quantity $-2 \pi \log 2 D N^{5}$ in the lower bound (A. 23) is not optimal at all. We were just looking for a lower bound $2 \pi\left|\sum_{i=1}^{N} d_{i}\right| \log \frac{R_{2}-R_{1}}{\eta}$ modulo a term which does not depend on $\eta$.

\section{Proof of Lemma A.6.}

Let $d=\sum_{i=1}^{N} d_{i}$. suppose $d>0$. We are going to prove by induction on $n$ the following proposition:

Under the previous hypothesis for $n \leqslant d$ we have

$$
\int_{\Omega_{\eta}}|\nabla u|^{2} \geqslant 2 \pi n \log \frac{R_{2}-R_{1}}{\eta}-2 \pi \log 2 D N^{5}
$$

the case $n=1$.

We construct by induction on $i$ for $1 \leqslant i \leqslant i_{0} \leqslant N$, a sequence $\eta \leqslant r_{i} \leqslant$ $R_{2}-R_{1}$ such that

$$
\left\{\begin{array}{l}
1-\quad r_{i}=\eta \text { if } B_{\eta}\left(a_{i}\right) \subset B_{r_{k}}\left(a_{k}\right) \text { for some } k<i \\
2-\quad r_{i} \text { is the largest real number such that } \\
r_{i} \leqslant R_{2}-R_{1}, \\
\partial B_{r_{i}}\left(a_{i}\right) \cap\left(\cup_{j>i} B_{\eta}(a j) \cup_{k<i} B_{r_{k}}\left(a_{k}\right)\right)=\emptyset, \\
\forall \eta \leqslant r<r_{i} \text { for which } \partial B_{r}\left(a_{i}\right) \cap\left(\cup_{j>i} B_{\eta}\left(a_{j}\right) \cup_{k<i} B_{r_{k}}\left(a_{k}\right)\right)=\emptyset \\
\text { we have } \operatorname{deg}\left(u, \partial B_{r}\left(a_{i}\right)\right) \neq 0 \\
\text { and either } \operatorname{deg}\left(u, \partial B_{r_{i}}\left(a_{i}\right)\right)=0 \text { or } r_{i}=R_{2}-R_{1}
\end{array}\right.
$$

we stop the construction of the sequence when we obtain an $i=i_{0}$ for which $r_{i_{0}}=R_{2}-R_{1}$.

It is clear that there exists some $i_{0} \in[1, N]$ such that $r_{i_{0}}=R_{2}-R_{1}$. Indeed $\left(B_{r_{i}}\left(a_{i}\right)\right)_{1 \leqslant i \leqslant i_{0}}$ is a union of disks which are either disjoint, or one nested in the other. Moreover if $B_{r_{i}}\left(a_{i}\right)$ is not contained in an other $B_{r_{k}}\left(a_{k}\right)$ we have for $i<i_{0}$

$$
\operatorname{deg}\left(u, \partial B_{r_{i}}\left(a_{i}\right)\right)=0
$$

thus $\cup_{i<i_{0}} B_{r_{i}}\left(a_{i}\right)$ is a union of disjoint disks such that the degree of $u$ on each component of the total boundary is equal to zero. Since $\sum_{i=1}^{N} d_{i}=d>0$, for topological reasons, it would not be possible to enclose all our balls $B_{\eta}\left(a_{i}\right)$ in a neutral cluster (i.e. a domain $\omega$ such that $\operatorname{deg}(u, \partial \omega)=0$ ), thus such an $i_{0} \leqslant N$ for which $r_{i_{0}}=R_{2}-R_{1}$ exists.

We introduce some notations for $1 \leqslant i \leqslant i_{0}$. Let $I_{i}$ be the following set

$$
I_{i}=\left\{\eta \leqslant r<r_{i} \text { s.t. } \partial B_{r}\left(a_{i}\right) \cap\left(\cup_{j>i} B_{\eta}\left(a_{i}\right) \cup_{k<i} B_{r_{k}}\left(a_{k}\right)\right)=\emptyset\right\}
$$

and we have

$$
\text { if } r_{i} \neq \eta \quad \forall r \in I_{i} \quad \operatorname{deg}\left(u, \partial B_{r}\left(a_{i}\right)\right) \neq 0
$$


Let $E_{i}$ and $F_{i}$ be the following sets

$$
\text { for } i \leqslant i_{0} \quad E_{i}=\left\{l \neq i \quad \text { s.t. } \quad B_{r_{l}}\left(a_{l}\right) \subset B_{r_{i}}\left(a_{i}\right)\right\} \quad,
$$

and

$$
\text { for } i \leqslant i_{0} \quad F_{i}=\left\{\begin{array}{l}
l \neq i \text { s.t. } B_{r_{l}}\left(a_{l}\right) \subset B_{r_{i}}\left(a_{i}\right) \text { and } \\
\forall l^{\prime} \neq l, l^{\prime}<i \quad B_{r_{l}}\left(a_{l}\right) \not \subset B_{r_{l^{\prime}}}\left(a_{l^{\prime}}\right)
\end{array}\right\} .
$$

We clearly have

$$
E_{i}=\bigcup_{l \in F_{i}} E_{l} \cup\{l\}
$$

and moreover

$$
\forall l \neq l^{\prime} \in F_{i} \quad\left(E_{l} \cup\{l\}\right) \cap\left(E_{l^{\prime}} \cup\left\{l^{\prime}\right\}\right)=\emptyset .
$$

Thus we have for any $i \leqslant i_{0}$

$$
\begin{aligned}
& \int_{B_{r_{i}}\left(a_{i}\right) \cap \Omega_{\eta}}|\nabla u|^{2} \geqslant \int_{I_{i}} d r \int_{\partial B_{r_{i}}}|\nabla u|^{2}+\sum_{j \in E_{i}} \int_{I_{j}} d r \int_{\partial B_{r}}|\nabla u|^{2} \\
& =\int_{I_{i}} d r \int_{\partial B_{r}}|\nabla u|^{2}+\sum_{j \in F_{i}}\left[\int_{I_{j}} d r \int_{\partial B_{r}}|\nabla u|^{2}\right. \\
& \left.+\sum_{k \in E_{j}} \int_{I_{k}} d r \int_{\partial B_{r}}|\nabla u|^{2}\right]
\end{aligned}
$$

Let us prove by induction for $i \leqslant i_{0}$ that

$$
\begin{aligned}
\int_{I_{i}} d r \int_{\partial B_{r}}|\nabla u|^{2}+\sum_{j \in E_{i}} \int_{I_{j}} d r \int_{\partial B_{r}}|\nabla u|^{2} & \geqslant 2 \pi \log \frac{r_{i}}{\eta}- \\
& -2 \pi \log 4 \# E_{i} .
\end{aligned}
$$

Suppose (A. 29) is true for $i \leqslant l-1$. Thus we have

$$
\left|I_{l}\right| \geqslant\left|r_{l}-\eta\right|-\sum_{k \in F_{l}} 2 r_{k}
$$

For $r \in I_{l}$ we have $\operatorname{deg}\left(u, \partial B_{r}\left(a_{l}\right)\right) \neq 0$, thus, this implies

$$
\forall r \in I_{l} \quad \int_{\partial B_{r}\left(a_{l}\right)}|\nabla u| \geqslant 2 \pi \quad
$$

and by Cauchy-Schwartz inequality we get

$$
\forall r \in I_{l} \quad \int_{\partial B_{r}\left(a_{l}\right)}|\nabla u|^{2} \geqslant \frac{2 \pi}{r} .
$$

Combining (A. 32) and (A. 30) we have

$$
\begin{aligned}
\int_{I_{l}} d r \int_{\partial B_{r}}|\nabla u|^{2} & \geqslant \int_{\eta+\sum_{k \in F_{l}} 2 r_{k}}^{r_{l}} \frac{2 \pi}{r} d r \\
& =2 \pi \log \frac{r_{l}}{\eta}-2 \pi \log \left(1+\sum_{k \in F_{l}} 2 \frac{r_{k}}{\eta}\right)
\end{aligned}
$$


Combining (A. 28) for $i=l$, (A. 33) and the fact that (A. 29) is true for $i \leqslant l-1$ we have

$$
\begin{aligned}
& \int_{I_{l}} \int_{\partial B_{r}}|\nabla u|^{2}+\sum_{j \in E_{l}} \int_{I_{j}} \int_{\partial B_{r}}|\nabla u|^{2} \geqslant \\
& \int_{I_{l}} \int_{\partial B_{r}}|\nabla u|^{2}+\sum_{k \in F_{l}}\left[2 \pi \log \frac{r_{k}}{\eta}-2 \pi \log 4 \# E_{k}\right] \geqslant 2 \pi \log \frac{r_{l}}{\eta} \\
& -2 \pi \log \left[1+\sum_{k \in F_{l}} \frac{2 r_{k}}{\eta}\right]+2 \pi \log \left[\prod_{k \in F_{l}}\right]\left(\frac{r_{k}}{\eta}\right)-2 \pi \log 4 \sum_{k \in F_{l}} \# E_{k} .
\end{aligned}
$$

Since $r_{k} \geqslant \eta$ we clearly have that

$$
1+\sum_{k \in F_{l}} \frac{2 r_{k}}{\eta} \leqslant 4 \# F_{l} \prod_{k \in F_{l}} \frac{r_{k}}{\eta}
$$

Thus (A. 34) yields

$$
\begin{aligned}
\int_{I_{l}} \int_{\partial B_{r}}|\nabla u|^{2}+\sum_{j \in E_{l}} \int_{I_{j}} \int_{\partial B_{r}}|\nabla u|^{2} \geqslant & 2 \pi \log \frac{r_{l}}{\eta}-2 \pi \log \left(4 \# F_{l}\right) \\
& -2 \pi \log 4 \sum_{k \in F_{l}} \# E_{k} .
\end{aligned}
$$

From (A. 26) and (A. 27) we deduce that

$$
\# E_{l}=\# F_{l}+\sum_{k \in F_{l}} \# E_{k}
$$

Combining (A. 35) and (A. 36) we obtain that the proposition is true for $i=l$, thus (A. 29) holds for $i \leqslant i_{0}$.

Applying inequality (A. 29) for $i=i_{0}$, since $r_{i_{0}}=R_{2}-R_{1}$, we obtain (A. 24) for $d \leqslant 1=n$.

\section{The case $n>1$}

Suppose (A. 24) is true for any positive integer $n \leqslant n^{\prime}$. Take a configuration of $\left(a_{i}, d_{i}\right)_{1 \leqslant i \leqslant N}$ such that $\sum_{i=1}^{N} d i \leqslant n^{\prime}+1$.

If $N=1$ the proof of (A. 24) for $n^{\prime}+1$ is straightforward. Suppose now $N>1$ and let

$$
L=\max _{i \neq i^{\prime}}\left|a_{i}-a_{i^{\prime}}\right|
$$

Esaim : CoCv June 1996, Vol.1, PP. 77-167 
we can always assume that $\left|a_{1}-a_{2}\right|=L$. We clearly have

$$
\begin{aligned}
\int_{\Omega_{\eta} \backslash L+\eta\left(a_{i}\right)}|\nabla u|^{2} & \geqslant \int_{L+\eta}^{R_{2}-R_{1}} \int_{\partial B_{r}}|\nabla u|^{2} \\
& \geqslant 2 \pi\left(n^{\prime}+1\right)^{2} \log \frac{R_{2}-R_{1}}{L+\eta} . \\
& \geqslant 2 \pi\left(n^{\prime}+1\right) \log \frac{R_{2}-R_{1}}{L+\eta}
\end{aligned}
$$

If $L \leqslant 2^{2\left(N^{2}+1\right)} \eta, \log \frac{R_{2}-R_{1}}{L+\eta} \geqslant \log \frac{R_{2}-R_{1}}{\eta}-2\left(N^{2}+2\right) \log 2$ and since $N \geqslant 2$ (A. 37) directly implies (A. 24 ) for $d \geqslant n^{\prime}+1$. If $L \geqslant 2^{2\left(N^{2}+1\right)} \eta$, there exists necessarily $k_{1} \in[1,2 N]$ such that

$$
\forall i \quad B_{\eta}\left(a_{i}\right) \cap\left[B_{2^{-k_{1} L}}\left(a_{1}\right) \backslash B_{2^{-k_{1}-1} L}\left(a_{1}\right)\right]=\emptyset
$$

In fact, since $\eta \leqslant 2^{-2 N^{2}} L$ each $B_{\eta}\left(a_{i}\right)$ for $i=2, \ldots, N$ can intersect at most two $B_{2-k}$ for $k \in[1,2 N]$.

In the same way, by induction for any $j \leqslant N$ such that $a_{j} \notin \cup_{i \leqslant j-1} B_{2-k_{i} L}\left(a_{i}\right)$ we construct $k_{j} \in[2 j N ; 2(j+1) N]$ verifying

$$
\forall i \quad B_{\eta}\left(a_{i}\right) \cap\left[B_{2^{-k_{j} L}}\left(a_{j}\right) \backslash B_{2^{-k_{j}-1} L}\left(a_{j}\right)\right]=\emptyset
$$

and we have

$$
\forall i<j \quad B_{\frac{3}{2} 2^{-k_{i} L}}\left(a_{i}\right) \cap B_{2^{-k_{j}}{ }}\left(a_{j}\right)=\emptyset
$$

We are lead to introduce the following notation, $C(a, s, r)$ denotes the annulus for $s \geqslant 1, r \leqslant \eta$ and $a \in B_{R_{1}}(0)$

$$
C(a, s, r)=B_{s r}(a) \backslash B_{r}(a)
$$

We have constructed disjoint annuli $C\left(a_{j}, \frac{3}{2}, 2^{-k_{j}-1} L\right)$ for some $j$ in $[1, N]$ such that $k_{j} \leqslant 2 N^{2}$ and the balls $B_{\eta}\left(a_{k}\right)$ are included in the $B_{2^{-k_{j}-1} L}\left(a_{j}\right)$. The sum of the degrees of $u$ on $\partial B_{2^{-k_{j}-1} L}\left(a_{j}\right)$ is equal to $d \geqslant n^{\prime}+1$.

Suppose there is exactly one of those $j=j_{1}$ such that $\operatorname{deg}\left(u, \partial B_{2^{-k_{j_{1}}-1} L}\left(a_{j_{1}}\right)\right) \neq 0$, then we must have

$$
\operatorname{deg}\left(u, \partial B_{2}^{-k_{j_{1}}-1} L\left(a_{j_{1}}\right)\right)=d
$$

In that case we repeat the procedure in $B_{2^{k j_{1}-1} L}\left(a_{j_{1}}\right)$. That is, let $R_{2,2}=$ $3 / 22^{-k_{j_{1}}-1} L, R_{1,2}=2^{-k_{j_{1}}-1} L$ and $N_{2}$ be the number of $a_{k}$ in $B_{R_{2,2}}\left(a_{j_{1}}\right)$.

If $N_{2}=1$. 
We clearly have

$$
\begin{aligned}
\int_{\Omega_{\eta} \backslash B_{\eta}\left(a_{j_{1}}\right)}|\nabla u|^{2} & \geqslant 2 \pi\left(n^{\prime}+1\right)^{2} \log \frac{R_{2,2}-R_{1,2}}{\eta} \\
& \geqslant 2 \pi\left(n^{\prime}+1\right) \log \frac{L}{\eta}-\left(k_{j_{1}}+2\right) \log 2\left(n^{\prime}+1\right) \\
& \geqslant 2 \pi\left(n^{\prime}+1\right) \log \frac{L}{\eta}-2\left(N^{2}+1\right) \log 2\left(n^{\prime}+1\right)
\end{aligned}
$$

and combining (A. 38) with (A. 37) we get the result.

If $N_{2}>1$.

Let $L_{2}$ be the following length

$$
L_{2}=\max _{i \neq i^{\prime} a_{i}, a_{i^{\prime}} \in B_{R_{2,2}}\left(a_{j_{1}}\right)}\left\{\left|a_{i}-a_{i^{\prime}}\right|\right\}=\left|a_{i_{2}}-a_{i_{2}^{\prime}}\right|
$$

and we have

$$
\begin{aligned}
\int_{B_{R_{2,2}}\left(a_{j_{1}}\right) \backslash B_{L_{2}+\eta}\left(a_{i_{2}}\right)}|\nabla u|^{2} & \geqslant \int_{L_{2}+\eta}^{R_{2,2}-R_{1,2}} \int_{\partial B_{r}}|\nabla u|^{2} \\
& \geqslant 2 \pi\left(n^{\prime}+1\right)^{2} \log \frac{R_{2,2}-R_{1,2}}{L_{2}+\eta} \\
& \geqslant 2 \pi\left(n^{\prime}+1\right) \log \frac{R_{2,2}-R_{1,2}}{L_{2}+\eta}
\end{aligned}
$$

If $L_{2} \leqslant 2^{2 N_{2}^{2}+2} \eta$.

In this case we have

$$
\log \frac{R_{2,2}-R_{1,2}}{L_{2}+\eta} \geqslant \log \frac{L}{\eta}-\left(k_{j_{1}}+4+2 N_{2}^{2}\right) \log 2
$$

but $\left(k_{j_{1}}+4+2 N_{2}^{2}\right) \geqslant 2\left(N^{2}+N_{2}^{2}+2\right)$ and since $N \leqslant\left(N_{2}+1\right)$ and $N_{2} \geqslant 2$ we easily get that $2\left(N^{2}+N_{2}^{2}+2\right)<N^{4}$. Finally combining (A. 39) and (A. 37 ) we get the desired result.

If $L_{2}>2^{2 N_{2}^{2} \eta}$

As previously we construct disjoint annuli $C\left(a_{j}, \frac{3}{2}, 2^{-k_{j}-1} L_{2}\right)$ for some $j$ in $[1, N]$ such that $a_{j} \in B_{R_{2,2}}\left(a_{j_{1}}\right)$ and $k_{j} \leqslant 2 N_{2}^{2}$ in such a way that all the $B_{\eta}\left(a_{k}\right)$ in $B_{R_{2,2}}\left(a_{j_{1}}\right)$ are included in the $B_{2^{-k_{j}-1} L_{2}}\left(a_{j}\right)$.

As before the sum of the degrees of $v$ on the $\partial B_{2^{-k_{j}-1} L_{2}}\left(a_{j}\right)$ is equal to $d \geqslant n^{\prime}+1 \ldots$.

In this way we construct by induction for $l \leqslant p$ the following sequences together

$$
\begin{aligned}
& N_{l} \in \mathbb{N}^{*} \quad \text { s.t. } \quad N_{l-1} \leqslant N_{l}+1 \\
& \qquad a_{j_{l}} \in\left\{a_{1}, \ldots, a_{N}\right\} \\
& \text { Esaim : Cocv June 1996, Vol.1, PP. } 77-167
\end{aligned}
$$


$k_{j_{l}} \in \mathbb{N}^{*}$ also denoted $k_{l}$ s.t. $\quad k_{l} \leqslant 2 N_{l}^{2}$

$$
L_{l}>0 \quad \text { s.t. } \quad L_{l} \leqslant 2^{-k_{l-1}-1} L_{l-1}
$$

and s.t. for any $l^{\prime}>l \quad B_{L_{l}}\left(a_{j_{l^{\prime}}}\right) \subset B_{R_{1, l}}\left(a_{j_{l}}\right)$

where $\quad R_{1, l}=2^{-k_{l-1}-1} L_{l-1} \quad$ and $\quad R_{2, l}=\frac{3}{2} R_{1, l}$

and finally such that

$$
\int_{B_{R_{2, l}}\left(a_{j_{l}-1}\right) \backslash B_{L_{l}}\left(a_{j_{l}}\right)}|\nabla u|^{2} \geqslant 2 \pi\left(n^{\prime}+1\right) \log \frac{R_{2, l}-R_{1, l}}{L_{l}+\eta}
$$

We stop the construction at $l=p$ when one of the following happens.

Let $N_{p}$ be the number of $a_{k}$ in $B_{R_{1, p}}\left(a_{j_{p-1}}\right)$

$N_{p}=1$

In this case we have

$$
\int_{B_{R_{2, p}}\left(a_{j_{p-1}}\right) \backslash B_{\eta}\left(a_{j_{p-1}}\right)}|\nabla u|^{2} \geqslant 2 \pi(n+1)^{2} \log \frac{R_{2, p}-R_{1, p}}{\eta}
$$

Summing (A. 40) for $l \leqslant p-1$, since the $B_{R_{2, l}}\left(a_{j_{l-1}}\right) \backslash B_{L_{l}}\left(a_{j_{l}}\right)$ are disjoint, we get

$$
\begin{aligned}
& \int_{\Omega_{\eta} \backslash B_{R_{2, p}}\left(a_{j_{p-1}}\right)}|\nabla u|^{2} \geqslant 2 \pi\left(n^{\prime}+1\right) \log \prod_{l \geqslant p-1} \frac{R_{2, l}-R_{1, l}}{L_{l}+\eta} \\
& \geqslant 2 \pi\left(n^{\prime}+1\right) \log \frac{R_{2}-R_{1}}{L_{p-1}+\eta}-2 \pi\left(n^{\prime}+1\right) \log 2 \sum_{l=1}^{p-2}\left(2+k_{l}\right) \\
& \geqslant 2 \pi\left(n^{\prime}+1\right) \log \frac{R_{2}-R_{1}}{L_{p-1}+\eta}-2 \pi\left(n^{\prime}+1\right) \log 2 \times 2 \sum_{l=1}^{p-2}\left(1+N_{l}^{2}\right) .
\end{aligned}
$$

Adding, now (A. 41) and (A. 42) we have

$$
\begin{aligned}
\int_{\Omega_{\eta}}|\nabla u|^{2} & \geqslant 2 \pi\left(n^{\prime}+1\right) \log \frac{R_{2}-R_{1}}{\eta}- \\
& -2 \pi\left(n^{\prime}+1\right) \log 2 \times 2 \sum_{l=1}^{p-1}\left(1+N_{l}^{2}\right) .
\end{aligned}
$$

Moreover $2 \sum_{l=1}^{p-1}\left(1+N_{l}^{2}\right) \leqslant 2(p-1)\left(1+N^{2}\right) \leqslant 2(N-1)\left(1+N^{2}\right)$

Since we are dealing with $N \geqslant 3(N=1$ or 2 have already been considered), we have $2 N^{3}-2\left(N^{2}-N+1\right)<N^{4}$ and we get the desired result because $n^{\prime}+1 \leqslant N D$.

$N_{p}>1$. Let $L_{p}$ be the following length

$$
L_{p}=\max _{i \neq i^{\prime}} \max _{a_{i}, a_{i^{\prime}} \in B_{R_{2, p}}\left(a_{j_{p}-1}\right)}\left\{\left|a_{i}-a_{i^{\prime}}\right|\right\}=\left|a_{i_{p}}-a_{i^{\prime} p}\right| .
$$

We have (A. 40) for $l=p$ 
Case 1: $L_{p} \leqslant 2^{2 N^{2}+2} \eta$

In this case we have

$$
\begin{aligned}
& \int_{B_{R_{2, p}}\left(a_{j_{p}-1}\right) \backslash B_{L_{p}}\left(a_{j_{p}-1}\right)}|\nabla u|^{2} \geqslant 2 \pi\left(n^{\prime}+1\right) \log 2^{-\left(k_{p-1}+2\right)} \frac{L_{p-1}}{L_{p+\eta}} \\
& \geqslant 2 \pi\left(n^{\prime}+1\right) \log \frac{L_{p-1}}{\eta}-2 \pi\left(n^{\prime}+1\right) \log 2 \times 2\left(N_{p}^{2}+N_{p-1}^{2}+2\right)
\end{aligned}
$$

Combining (A. 44) and (A. 42) we obtain

$$
\begin{aligned}
\int_{\Omega_{\eta}}|\nabla u|^{2} & \geqslant 2 \pi\left(n^{\prime}+1\right) \log \frac{R_{2}-R_{1}}{\eta}- \\
& -2 \pi\left(n^{\prime}+1\right) \log 2 \times 2 \sum_{l=1}^{p}\left(1+N_{l}^{2}\right)
\end{aligned}
$$

Since $N_{p}>1, p<N, 2 \sum_{l=1}^{p}\left(1+N_{l}^{2}\right) \geqslant 2(N-1)\left(1+N^{2}\right)<N^{4}$, we get the result because $n^{\prime}+1 \geqslant N D$.

Case 2: $L_{p}>2^{2 N_{p}^{2}+2} \eta$

We construct, as before, disjoint annuli $\left(C\left(a_{p_{i}}, \frac{3}{2}, 2^{-k_{i}-1} L_{p}\right)\right)_{1 \leqslant i \leqslant n_{p}}$ (where $n_{p} \geqslant N_{p}$ and $\left.k_{i} \in\left[1,2 N_{p}^{2}\right]\right)$ such that all the $B_{\eta}\left(a_{k}\right)$ in $B_{R_{2, p}}\left(a_{j_{p-1}}\right)$ are included in the $B_{2-k_{i}-1} L_{p}\left(a_{p_{i}}\right)$. The sum of the degrees $d_{i}$ of $u$ on the circles $\partial B_{2^{-k_{i}-1} L_{p}}\left(a_{p_{i}}\right)$ is equal to $d \geqslant n^{\prime}+1$ and in this case there exists two disjoints $\partial B_{2^{-k_{i}-1} L_{p}}\left(a_{p_{i}}\right)$ such that $d_{i} \neq 0$ (otherwise we continue the previous construction).

We can apply the hypothesis (A. 24) for $n \leqslant n^{\prime}$ on the $B_{\frac{3}{2} 2^{-k_{i}-1} L_{p}}\left(a_{p_{i}}\right)$ and we have $\forall 1 \leqslant i \leqslant n_{p}$

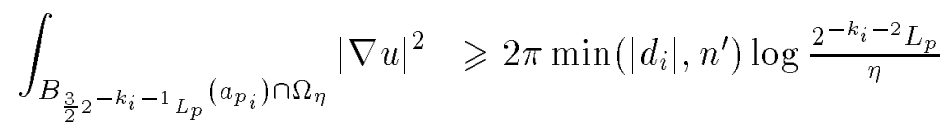

$$
\begin{aligned}
& -2 \pi \log 2 D\left(N_{p}^{i}\right)^{5},
\end{aligned}
$$

where $N_{p}^{i}$ is the number of $a_{k}$ in $B_{2^{-k_{i}-2} L_{p}}\left(a_{p_{i}}\right)$. In particular we have

$$
\sum_{i=1}^{n_{p}} N_{p}^{i}=N_{p}
$$

Since $\sum_{i=1}^{n_{p}}\left|d_{i}\right| \geqslant n^{\prime}+1$ and since there exists at least two $i$ such that $\left|d_{i}\right| \neq 0$ we have $\sum_{i=1}^{n_{p}} \min \left(\left|d_{i}\right| ; n^{\prime}\right) \geqslant n^{\prime}+1$ and summing (A. 46) for $i=1, \ldots, n_{p}$ we Esaim : Cocv June 1996, Vol.1, PP. 77-167 
get

$$
\begin{aligned}
\int_{B_{L_{p}}\left(a_{j_{p-1}}\right)}|\nabla u|^{2} & \geqslant 2 \pi\left(n^{\prime}+1\right) \log \frac{L_{p}}{\eta}- \\
& -2 \pi D \log 2\left[\sum_{i=1}^{n_{p}}\left[N_{p}^{i}\left(k_{i}+2\right)+\left(N_{p}^{i}\right)^{5}\right]\right]
\end{aligned}
$$

$$
\geqslant 2 \pi\left(n^{\prime}+1\right) \log \frac{L_{p}}{\eta} \quad-2 \pi D \log 2\left[2 N_{p}\left(N_{p}^{2}+1\right)+\sum_{i=1}^{n_{p}}\left(N_{p}^{i}\right)^{5}\right] .
$$

Combining (A. 42), the first line of (A. 44) and (A. 48) we obtain

$$
\begin{aligned}
& \int_{\Omega_{\eta}}|\nabla u|^{2} \geqslant 2 \pi\left(n^{\prime}+1\right) \log \frac{R_{2}-R_{1}}{\eta}- \\
& -2 \pi D \log 2\left[2 N \sum_{l=1}^{p-1}\left(1+N_{l}^{2}\right)+2 N_{p}\left(N_{p}^{2}+1\right)+\sum_{i=1}^{n_{p}}\left(N_{p}^{i}\right)^{5}\right]
\end{aligned}
$$

In the other hand we have the following bound

$$
\begin{aligned}
& \text { let } n \geqslant 2, \quad \text { and } \quad\left(\alpha_{i}\right)_{i=1 \ldots n} \quad \text { s.t. } \alpha_{i} \leqslant 1 \\
& \sum_{i=1}^{n} \alpha_{i}^{5} \leqslant\left(\sum_{i=1}^{n} \alpha_{i}\right)^{5}-2\left(\sum_{i=1}^{n} \alpha_{i}\right)^{3}-2 \sum_{i=1}^{n} \alpha_{i}
\end{aligned}
$$

Indeed let $l$ be such that $\alpha_{l}=\min _{i=1 \ldots n} \alpha_{i}$. Thus we have

$$
\begin{aligned}
\left(\sum_{i=1}^{n} \alpha_{i}\right)^{5} & \geqslant \alpha_{l}^{5}+\left(\sum_{i \neq l} \alpha_{i}\right)^{5}+C_{5}^{2} \alpha_{l}^{2}\left(\sum_{i \neq l} \alpha_{i}\right)^{3}+C_{5}^{1} \alpha_{l}^{4}\left(\sum_{i \neq l} \alpha_{i}\right) \\
& \geqslant \sum_{i=1}^{n} \alpha_{i}^{5}+\frac{5 \times 4 \times 3}{2}\left(\sum_{i \neq l} \alpha_{i}\right)^{3}+5\left(\sum_{i \neq l} \alpha_{i}\right)
\end{aligned}
$$

and since $\alpha_{l}=\min _{i=1 \ldots n} \alpha_{i}$ we have $\sum_{i \neq l} \alpha_{i} \geqslant \frac{n-1}{n} \sum_{i=1}^{n} \alpha_{i}$ and since $n \geqslant 2$ we have $\frac{n-1}{n} \leqslant \frac{1}{2}$, thus (A. 51) yields

$$
\left(\sum_{i=1}^{n} \alpha_{i}\right)^{5} \geqslant \sum_{i=1}^{n} \alpha_{i}^{5}+\frac{5 \times 4 \times 3}{2^{4}}\left(\sum_{i=1}^{n} \alpha_{i}\right)^{3}+\frac{5}{2} \sum_{i=1}^{n} \alpha_{i}
$$

which implies (A. 50). Combining (A. 50) and (A. 49) we obtain

$$
\begin{aligned}
\int_{\Omega_{\eta}}|\nabla u|^{2} & \geqslant 2 \pi\left(n^{\prime}+1\right) \log \frac{R_{2}-R_{1}}{\eta}- \\
& -2 \pi D \log 2\left[2 N(p-1)\left(1+N^{2}\right)+N_{p}^{5}\right],
\end{aligned}
$$

but $p-1 \leqslant N-N_{p}$ thus $2 N(p-1)\left(1+N^{2}\right)+N_{p}^{5} \leqslant 2 N\left(N-N_{p}\right)\left(1+N^{2}\right)+N_{p}^{5}$ and since $N_{p} \geqslant 2$ one verifies that

$$
N^{5} \geqslant 2 N\left(N-N_{p}\right)\left(1+N^{2}\right)+N_{p}^{5}
$$

and (A. 52) implies

$$
\int_{\Omega_{\eta}}|\nabla u|^{2} \geqslant 2 \pi\left(n^{\prime}+1\right) \log \frac{R_{2}-R_{1}}{\eta}-2 \pi D \log 2 N^{5} .
$$


This is the desired result.

The author thanks F. Bethuel for very interesting discussions on this subject.

\section{REFERENCES}

[1] L. Almeida and F. Bethuel: Méthodes topologiques pour l'équation de GinzburgLandau, C.R.A.S, Paris, 320, 935-939, 1995.

[2] F. Bethuel, H. Brezis and F. Hélein: Asymptotics for the minimization of a GinzburgLandau functional, Calculus of variations and PDE1, 123-148, 1993.

[3] F. Bethuel, H. Brezis and F. Hélein: Ginzburg-Landau vortices, Birkhaüser, 1994.

[4] F. Bethuel and T. Rivière: Vortices for a variational problem related to supraconductivity, Ann. Inst. Henri Poincaré, (analyse non linéaire), 12, 3, 243-303, 1995.

[5] R. Bott and L. Tu: Differential forms in Algebraic Topology, Springer, 1986.

[6] H. Brezis, J.-M Coron and E. Lieb: Harmonic maps with defects, Comm. Math. Phys., 107, 649-705, 1986.

[7] H. Brezis, F. Merle and T. Rivière: Quantization effects for $-\Delta u=u\left(1-|u|^{2}\right)$ in $\mathbb{R}^{2}$, to appear in Arch. for rat. Mech. Analysis.

[8] J. Fröhlich and M. Struwe: Variational problems on vector bundles, Commun. Math. Phys., 131, 431-464, 1990.

[9] R. Hardt and L. Simon: Seminar on geometric Measure Theory, Birkhaüser, 1986.

[10] A. Jaffe and C. Taubes: Vortices and Monopoles, Birkhäuser, 1980.

[11] F.H.Lin: Solutions of Ginzburg-Landau equations and critical points of the renormalized energy, Ann. Inst. Henri Poincaré, (analyse non linéaire),12, 5, 599-622, 1995.

[12] T. Rivière: Lignes de tourbillon dans le modèle abelien de Higgs, C.R.A.S., Paris, $321,73-76,1995$. 